\title{
Simple, Stable and Versatile Double-Allylation Reagents for the Stereoselective Preparation of Skeletally Diverse Compounds
}

\author{
Feng Peng and Dennis G. Hall* \\ Department of Chemistry, University of Alberta, Edmonton, AB, T6G 2G2, Canada
}

\section{Supplementary Information}

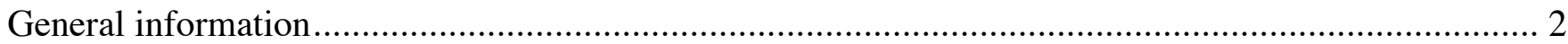

Determination of enantiomeric excess, diastereoselectivity and $\mathrm{E} / \mathrm{Z}$ ratio................................... 2

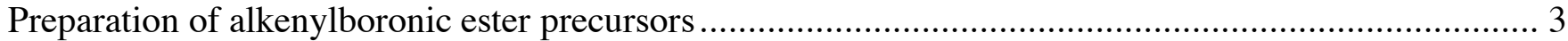

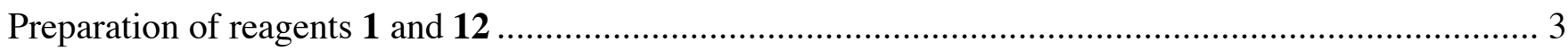

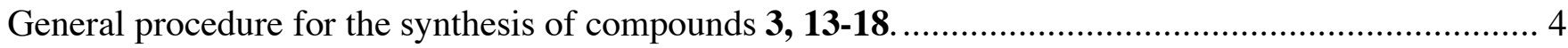

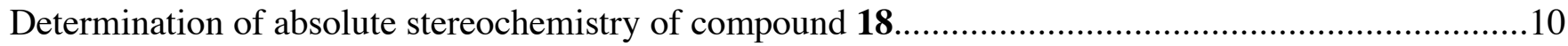

General procedure for the synthesis of compounds $\mathbf{5 - 8}$ and additional examples $\mathbf{2 1}, \mathbf{2 2} \ldots \ldots \ldots \ldots \ldots \ldots . . . . .11$

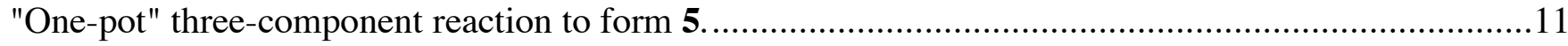

General procedure for the synthesis of compounds 9-11 and additional example 23......................14

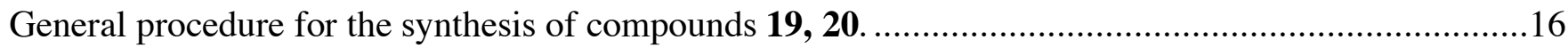

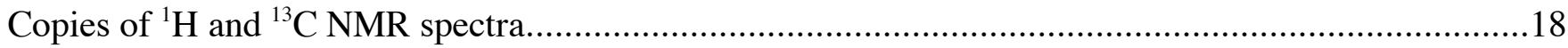

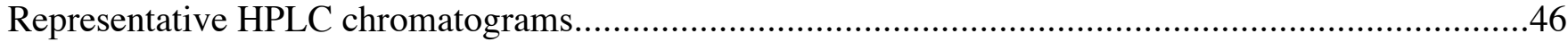

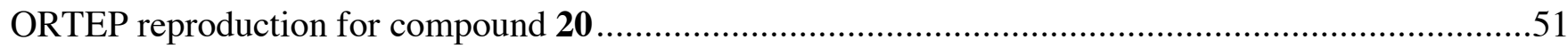

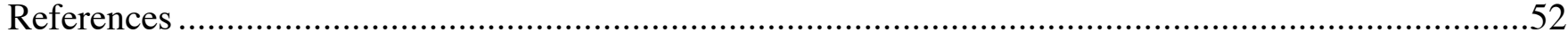




\section{General information}

Unless otherwise noted, all reactions were performed under an argon atmosphere using flame-dried glassware. Toluene, hexanes and $\mathrm{CH}_{2} \mathrm{Cl}_{2}$ were distilled over $\mathrm{CaH}_{2}$. THF and $\mathrm{Et}_{2} \mathrm{O}$ were distilled over sodium/benzophenone ketyl. All aldehydes were purified by Kugelrohr distillation prior to use. Thin layer chromatography (TLC) was performed on Silica Gel 60 F254 plates and were visualized with UV light and $\mathrm{KMnO}_{4}$ stain. NMR spectra were recorded on 300,400 or $500 \mathrm{MHz}$ instruments. The residual solvent protons $\left({ }^{1} \mathrm{H}\right)$ or the solvent carbons $\left({ }^{13} \mathrm{C}\right)$ were used as internal standards. Boron NMR spectra are referenced to external $\mathrm{BF}_{3} \cdot \mathrm{OEt}_{2} \cdot{ }^{1} \mathrm{H}$ NMR data are presented as follows: chemical shift in ppm downfield from tetramethylsilane (multiplicity, coupling constant, integration). The following abbreviations are used in reporting NMR data: s, singlet; br s, broad singlet; d, doublet; t, triplet; q, quartet; qt, quartet of triplets; dd, doublet of doublets; dt, doublet of triplets; $\mathrm{AB}, \mathrm{AB}$ quartet; m, multiplet. High resolution mass spectra were recorded by the University of Alberta Mass Spectrometry Services Laboratory using either electron impact (EI) or electrospray (ES) ionization techniques. Infrared spectra and optical rotations were recorded by University of Alberta Spectral Services and combustion analyses were performed by the University of Alberta Micro-Analytical Lab. X-ray diffraction data were collected by the University of Alberta X-Ray Crystallography Laboratory.

\section{Determination of enantiomeric excess, diatereoselectivity and $E / Z$ ration.}

The enantiomeric excesses for all the compounds were determined using an HP 1100 HPLC system. The enantiomeric excesses for compounds $\mathbf{3 b}, \mathbf{3 c}, \mathbf{3 g}$ and $\mathbf{3 h}$ were determined on the corresponding phenylisocyanate adduct using an HP 1100 HPLC system. The enantiomeric excesses for compounds 9 and 23 were determined on the corresponding alcohols (By transforming the alkene to alcohol ${ }^{1}$ ) using an HP 1100 HPLC system. The enantiomeric excesses for compounds $\mathbf{1 0}$ and $\mathbf{1 9}$ were determined on the corresponding alcohol phenylisocyanate adduct (By transforming the alkene to alcohol ${ }^{1}$ ) using an HP 1100 HPLC system. Details of chromatographic conditions are indicated under each compound. Columns Chiralcel AD-RH, Chiralcel OD-RH, Chiralcel OD, and Chiralcel AD were purchased from Chiral Technologies Inc.. Racemic compounds were prepared in the same manner using the pinacol boronate derivatives. The diatereoselectivity for compounds 5, 6, 7, 8, 9, 10, 11, 19, 20, 21 and 22 were determined by ${ }^{1} \mathrm{H}$ NMR. The $\mathrm{E} / \mathrm{Z}$ ratios for compounds $3, \mathbf{1 3}, \mathbf{1 4}, \mathbf{1 5}, \mathbf{1 6}, 17$ and 18 were determined by ${ }^{1} \mathrm{H}$ NMR using TMS peaks of crude mixtures. 


\section{Preparation of alkenylboronic ester precursors}

A solution of representative the alkenylboronic acids ( $E$ and Z-1-propenylboronic acids were purchased from Aldrich, and vinyl boronic acid was prepared according to a reported procedure $\left.{ }^{2}\right)(6 \mathrm{mmol})$ and $(1 R, 2 R, 3 S, 5 R)-(-)$-pinanediol $(5 \mathrm{mmol})$ in $10 \mathrm{ml}$ of anhydrous THF was stirred $12 \mathrm{~h}$ at room temperature. The solvent was then removed and the product was purified by flash chromatography (78\% yield) to obtain analytically pure alkenyl boronate ester, which had identical spectroscopic data to that reported. ${ }^{2}$

\section{Preparation of reagents 1 and $12 .^{3}$}

A solution of $0.5 \mathrm{~mL}$ of dichloromethane in $10 \mathrm{ml}$ of anhydrous THF was cooled to $-100^{\circ} \mathrm{C}$ using $95 \%$ ethanol/liquid nitrogen slush bath. $n$-BuLi $(3.33 \mathrm{~mL}, 5 \mathrm{mmol})$ was added through the inside wall of reaction flask over $5 \mathrm{~min}$. The reaction mixture was stirred for $20 \mathrm{~min}$ and then vinylboronic ester (5 mmol) in $3 \mathrm{~mL}$ of anhydrous THF was added in one portion. After $2 \mathrm{~min}$, the reaction mixture was warmed up to $0{ }^{\circ} \mathrm{C}$ and stirred for an additional $40 \mathrm{~min}$. At this stage, the reaction mixture was cooled down to $-78{ }^{\circ} \mathrm{C}$ and freshly prepared $\mathrm{TMSCH}_{2} \mathrm{MgBr}(5 \mathrm{mmol})$ in THF $(6 \mathrm{~mL})$ was added slowly. The reaction mixture was stirred $3 \mathrm{~h}$ at $-78{ }^{\circ} \mathrm{C}, 12 \mathrm{~h}$ at room temperature, then quenched with $\mathrm{NH}_{4} \mathrm{Cl}$ solution, extracted with ether, dried over anhydrous $\mathrm{Na}_{2} \mathrm{SO}_{4}$, filtered and purified by flash chromatography.

\section{Reagent 1}

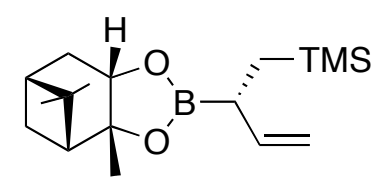

Flash chromatography (hexanes) yielded a colorless oil (1.06 g, 70\%).

TLC (25\% EtOAc/Hexane, $\mathrm{KMnO}_{4}$ ): Rf 0.90;

${ }^{1} \mathrm{H}$ NMR $\left(\mathrm{CDCl}_{3}, 400 \mathrm{MHz}\right): \delta 5.89(\mathrm{ddd}, J=16.8,10.0,8.4 \mathrm{~Hz}, 1 \mathrm{H}), 5.02(\mathrm{ddd}, J=17.2,1.6,1.6 \mathrm{~Hz}$, $1 \mathrm{H}), 4.90(\mathrm{~d}, J=10.0 \mathrm{~Hz}, 1 \mathrm{H}), 4.28(\mathrm{dd}, J=8.8,2.0 \mathrm{~Hz} 1 \mathrm{H}), 2.37-2.31(\mathrm{~m}, 1 \mathrm{H}), 2.22-2.18(\mathrm{~m}, 1 \mathrm{H})$, 2.08-2.06 (m, 1H), 1.99-1.97 (m, 1H), 1.92-1.82 (m, 2H), $1.40(\mathrm{~s}, 3 \mathrm{H}), 1.29(\mathrm{~s}, 3 \mathrm{H}), 1.13(\mathrm{~d}, J=10.8$ $\mathrm{Hz}, 1 \mathrm{H}), 0.88(\mathrm{dd}, J=14.4,7.6 \mathrm{~Hz}, 1 \mathrm{H}), 0.85(\mathrm{~s}, 3 \mathrm{H}), 0.70(\mathrm{dd}, J=14.8,7.6 \mathrm{~Hz}, 1 \mathrm{H}), 0.02(\mathrm{~s}, 9 \mathrm{H})$;

${ }^{13} \mathrm{C} \mathrm{NMR}\left(\mathrm{CDCl}_{3}, 100 \mathrm{MHz}\right): \delta 142.2,112.2,85.5,77.8,51.4,39.4,38.2,35.4,28.5$, 27.1, 26.2, 24.0, 17.0, -1.0;

${ }^{11} \mathrm{~B}$ NMR $\left(\mathrm{CDCl}_{3}, 128 \mathrm{MHz}\right) \delta 32.6$;

IR (neat): $\mathrm{cm}^{-1} 2950,1627,1386,1246,859$;

HRMS (EI, $m / z$ ) calcd for $\mathrm{C}_{17} \mathrm{H}_{31} \mathrm{O}_{2} \mathrm{BSi}$ : 306.2186; found: $306.2181\left[\mathrm{M}^{+}\right]$. 
$[\alpha]_{D}^{25}-18.98\left(\mathrm{c}=0.60, \mathrm{CHCl}_{3}\right)$.

\section{Reagent $Z-12$}

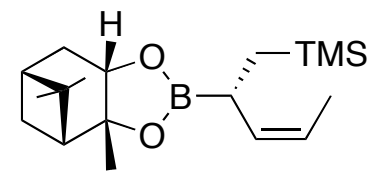

Flash chromatography (hexanes) yielded a colorless oil $(1.31 \mathrm{~g}, 85 \%)$.

TLC (25\% EtOAc/Hexane, $\left.\mathrm{KMnO}_{4}\right)$ : Rf 0.90;

${ }^{1} \mathrm{H}$ NMR $\left(\mathrm{CDCl}_{3}, 400 \mathrm{MHz}\right): \delta 5.40-5.30(\mathrm{~m}, 2 \mathrm{H}), 4.25(\mathrm{dd}, J=8.7,2.0 \mathrm{~Hz}, 1 \mathrm{H}), 2.36-2.28(\mathrm{~m}, 1 \mathrm{H})$,

2.24-2.14 (m, 2H), $2.04(\mathrm{dd}, J=5.3,5.3 \mathrm{~Hz}, 1 \mathrm{H}), 1.92-1.86(\mathrm{~m}, 1 \mathrm{H}), 1.82(\mathrm{ddd}, J=14.5,3.3,2.0 \mathrm{~Hz}$ $1 \mathrm{H}), 1.62(\mathrm{~d}, J=4.9 \mathrm{~Hz}, 1 \mathrm{H}), 1.38(\mathrm{~s}, 3 \mathrm{H}), 1.28(\mathrm{~s}, 3 \mathrm{H}), 1.12(\mathrm{~d}, J=10.9 \mathrm{~Hz}, 1 \mathrm{H}), 0.88(\mathrm{dd}, J=14.6$, $6.8 \mathrm{~Hz}, 1 \mathrm{H}), 0.86(\mathrm{~s}, 3 \mathrm{H}), 0.65(\mathrm{dd}, J=14.6,8.3 \mathrm{~Hz}, 1 \mathrm{H}),-0.02(\mathrm{~s}, 9 \mathrm{H})$;

${ }^{13} \mathrm{C} \mathrm{NMR}\left(\mathrm{CDCl}_{3}, 100 \mathrm{MHz}\right): \delta 134.5,121.4,85.3,77.7,51.5,39.4,38.2,35.5,28.5,27.1,26.1,24.0$, 18.2, 13.1, - 1.0;

IR (neat): $\mathrm{cm}^{-1}: 2917,1442,1375,1247,1078$;

HRMS (EI, $m / z$ ) calcd for $\mathrm{C}_{10} \mathrm{H}_{17} \mathrm{O}_{5} \mathrm{~B}: 320.2343$; found: $320.2341\left[\mathrm{M}^{+}\right]$.

$[\alpha]_{D}^{25}-9.79\left(\mathrm{c}=0.40, \mathrm{CHCl}_{3}\right)$.

\section{Reagent $E$-12}

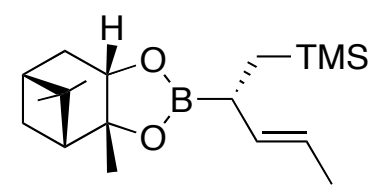

Flash chromatography (hexanes) yielded a colorless oil (1.25 g, 80\%).

TLC (25\% EtOAc/Hexane, $\left.\mathrm{KMnO}_{4}\right)$ : Rf 0.90;

${ }^{1} \mathrm{H}$ NMR $\left(\mathrm{CDCl}_{3}, 400 \mathrm{MHz}\right): \delta 5.46-5.34(\mathrm{~m}, 2 \mathrm{H}), 4.25(\mathrm{dd}, J=8.7,2.0 \mathrm{~Hz}, 1 \mathrm{H}), 2.36-2.28(\mathrm{~m}, 1 \mathrm{H})$, 2.22-2.14 (m, 2H), $2.04(\mathrm{dd}, J=5.2,5.2 \mathrm{~Hz}, 1 \mathrm{H}), 1.92-1.86(\mathrm{~m}, 2 \mathrm{H}), 1.82(\mathrm{ddd}, J=14.4,3.2,2.0 \mathrm{~Hz}$ $1 \mathrm{H}), 1.63(\mathrm{~d}, J=4.7 \mathrm{~Hz}, 1 \mathrm{H}), 1.38(\mathrm{~s}, 3 \mathrm{H}), 1.28(\mathrm{~s}, 3 \mathrm{H}), 1.12(\mathrm{~d}, J=10.8 \mathrm{~Hz}, 1 \mathrm{H}), 0.85(\mathrm{dd}, J=14.7$, $7.6 \mathrm{~Hz}, 1 \mathrm{H}), 0.84(\mathrm{~s}, 3 \mathrm{H}), 0.65(\mathrm{dd}, J=14.6,7.6 \mathrm{~Hz}, 1 \mathrm{H}),-0.02(\mathrm{~s}, 9 \mathrm{H})$;

${ }^{13} \mathrm{C}$ NMR $\left(\mathrm{CDCl}_{3}, 100 \mathrm{MHz}\right): \delta 134.5,122.9,85.4,77.7,51.4,39.4,38.2,35.5,28.5,27.1,26.1,24.0$, $18.0,17.8,-1.0$

IR (neat): $\mathrm{cm}^{-1}: 2919,1448,1377,1250,849$;

HRMS (EI, $m / z$ ) calcd for $\mathrm{C}_{10} \mathrm{H}_{17} \mathrm{O}_{5} \mathrm{~B}: 320.2343$; found: $320.2339\left[\mathrm{M}^{+}\right]$.

$[\alpha]_{D}^{25}-11.75\left(\mathrm{c}=0.45, \mathrm{CHCl}_{3}\right)$.

\section{General procedure for the synthesis of compounds 3, 13-18.}

At $-78{ }^{\circ} \mathrm{C}$, to a flame-dried round bottom flask, was added the boron reagent $(0.2 \mathrm{mmol})$, aldehyde $(0.2$ mmol) and $1 \mathrm{~mL}$ of dichloromethane. The mixture was stirred for $5 \mathrm{~min}$, after which $\mathrm{BF}_{3}-\mathrm{Et}_{2} \mathrm{O}(0.2$ mmol) was added. The reaction mixture was stirred further $12 \mathrm{~h}$ at $-78{ }^{\circ} \mathrm{C}$ and then quenched with an 
aqueous $\mathrm{NaHCO}_{3}$ solution, extracted with ether, dried over anhydrous $\mathrm{Na}_{2} \mathrm{SO}_{4}$ and filtered. The products were purified by flash chromatography.

\section{(R,E)-7-(trimethylsilyl)-1-phenylhept-5-en-3-ol (3a)}

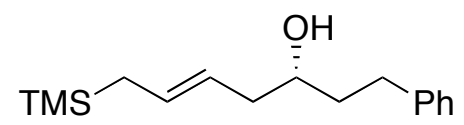

Flash chromatography (5\% EtOAc/hexanes) yielded a colorless oil (41 mg, 77\%).

TLC (25\% EtOAc/Hexane, $\left.\mathrm{KMnO}_{4}\right)$ : Rf 0.4;

${ }^{1} \mathrm{H}$ NMR $\left(\mathrm{CDCl}_{3}, 400 \mathrm{MHz}\right): \delta 7.32-7.16(\mathrm{~m}, 5 \mathrm{H}), 5.56(\mathrm{ddd}, J=15.6,8.1,8.1 \mathrm{~Hz}, 1 \mathrm{H}), 5.24(\mathrm{ddd}, J=$ 15.1, 7.3, 7.3 Hz, 1H), 3.60 (ddddd, $J=4.4,3.9,3.9,3.9,3.9 \mathrm{~Hz}, 1 \mathrm{H}), 2.86-2.64(\mathrm{~m}, 2 \mathrm{H}), 2.32-2.26(\mathrm{~m}$, $1 \mathrm{H}), 2.15-2.06(\mathrm{~m}, 1 \mathrm{H}), 1.82-1.74(\mathrm{~m}, 2 \mathrm{H}), 1.65(\mathrm{~d}, J=3.9 \mathrm{~Hz}, 1 \mathrm{H}), 1.48(\mathrm{~d}, J=8.1 \mathrm{~Hz}, 2 \mathrm{H}), 0.01(\mathrm{~s}$, 9H);

${ }^{13} \mathrm{C} \mathrm{NMR}\left(\mathrm{CDCl}_{3}, 100 \mathrm{MHz}\right): \delta 142.3,131.0,128.4,128.3,125.7,123.9,70.3,41.1,38.4,32.1,23.1$, 2.0;

IR (neat): $\mathrm{cm}^{-1} 3360,3026,2952,1604,1248$;

HRMS (ESP) calcd for $\mathrm{C}_{16} \mathrm{H}_{26} \mathrm{OSiNa}$ : 285.1645; found: 285.1643.

$[\alpha]_{D}^{25}+2.25\left(\mathrm{c}=1.10, \mathrm{CHCl}_{3}\right)$.

HPLC : Chiralcel OD, $10 \%$ PrOH/Hexane, $0.50 \mathrm{~mL} / \mathrm{min}$, UV detection at $210 \mathrm{~nm}$, , major peak at 15.6 min., minor peak at 12.5 min., $95 \%$ ee.

\section{$(R, E)-1-($ trimethylsilyl)dodec-2-en-5-ol (3b)}

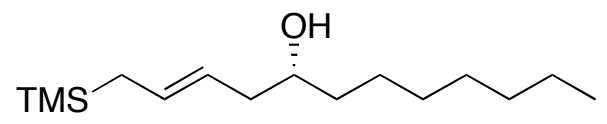

Flash chromatography (5\% EtOAc/hexanes) yielded a colorless oil (36 mg, 70\%), which gave satisfactory analytical data.

TLC (25\% EtOAc/ hexanes, $\left.\mathrm{KMnO}_{4}\right)$ : Rf 0.60;

${ }^{1} \mathrm{H}$ NMR $\left(\mathrm{CDCl}_{3}, 400 \mathrm{MHz}\right): \delta 5.54(\mathrm{ddd}, J=15.6,8.1,8.1 \mathrm{~Hz}, 1 \mathrm{H}), 5.26(\mathrm{ddd}, J=15.5,7.3,7.3 \mathrm{~Hz}$, $1 \mathrm{H}), 3.58$ (ddddd, $J=4.3,3.9,3.9,3.9,3.9 \mathrm{~Hz}, 1 \mathrm{H}), 2.28-2.20(\mathrm{~m}, 1 \mathrm{H}), 2.10-2.00(\mathrm{~m}, 1 \mathrm{H}), 1.60(\mathrm{~d}, J=$ $3.9 \mathrm{~Hz}, 1 \mathrm{H}), 1.48(\mathrm{~d}, J=8.2 \mathrm{~Hz}, 2 \mathrm{H}), 1.48-1.22(\mathrm{~m}, 10 \mathrm{H}), 0.88(\mathrm{dd}, J=6.7,6.7 \mathrm{~Hz}, 3 \mathrm{H}), 0.01(\mathrm{~s}, 9 \mathrm{H})$;

${ }^{13} \mathrm{C} \mathrm{NMR}\left(\mathrm{CDCl}_{3}, 100 \mathrm{MHz}\right): \delta 130.7,124.2,71.1,40.9,36.7,331.8,29.7,29.3,25.7,23.0,22.6,14.1$, 2.0;

IR (neat): $\mathrm{cm}^{-1} 3349,2955,2928,2856,1248$;

HRMS (ESP) calcd for $\mathrm{C}_{15} \mathrm{H}_{32} \mathrm{OSiNa}$ : 279.2115; found: 279.2113.

$[\alpha]_{D}^{25}-3.5\left(\mathrm{c}=0.9, \mathrm{CHCl}_{3}\right)$.

HPLC : Chiralcel OD, $10 \% i \mathrm{PrOH} / \mathrm{Hexane}, 0.50 \mathrm{~mL} / \mathrm{min}$., $\mathrm{UV}$ detection at $210 \mathrm{~nm}$, major peak at 15.9 min., minor peak at $12.2 \mathrm{~min} ., 98 \%$ ee.

\section{(S,E)-2-methyl-7-(trimethylsilyl)hept-5-en-3-ol (3c)}




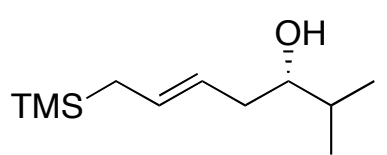

Flash chromatography (5\% EtOAc/Hexanes) yielded a colorless oil (25 mg, 63\%), which gave satisfactory analytical data.

TLC (15\% EtOAc/hexanes, $\left.\mathrm{KMnO}_{4}\right)$ : Rf 0.60;

${ }^{1} \mathrm{H} \mathrm{NMR}\left(\mathrm{CDCl}_{3}, 400 \mathrm{MHz}\right): \delta 5.54(\mathrm{ddd}, J=15.6,8.0,8.0 \mathrm{~Hz}, 1 \mathrm{H}), 5.26(\mathrm{ddd}, J=15.1,7.8,7.8 \mathrm{~Hz}$, $1 \mathrm{H}), 3.32-3.27(\mathrm{~m}, 1 \mathrm{H}), 2.30-2.23(\mathrm{~m}, 1 \mathrm{H}), 2.04$ (ddd, $J=13.9,8.5,8.5 \mathrm{~Hz}, 1 \mathrm{H}), 1.67$ (dddd, $J=13.4$, 6.7, 6.7, 6.7 Hz, 1H), $1.58(\mathrm{~d}, J=3.7 \mathrm{~Hz}, 1 \mathrm{H}), 1.48(\mathrm{~d}, J=8.1 \mathrm{~Hz}, 2 \mathrm{H}), 0.94(\mathrm{~d}, J=6.7 \mathrm{~Hz}, 3 \mathrm{H}), 0.92(\mathrm{~d}$, $J=6.7 \mathrm{~Hz}, 3 \mathrm{H}), 0.00(\mathrm{~s}, 9 \mathrm{H})$;

${ }^{13} \mathrm{C} \mathrm{NMR}\left(\mathrm{CDCl}_{3}, 100 \mathrm{MHz}\right): \delta 130.6,124.6,75.7,37.9,32.9,23.0,18.8,17.6,-2.0$;

IR (neat): $\mathrm{cm}^{-1} 3390,2956,1404,1248$;

HRMS (ESP) calcd for $\mathrm{C}_{15} \mathrm{H}_{32} \mathrm{OSiNa}$ : 223.1489; found: 223.1487.

$[\alpha]_{D}^{25}-8.55\left(\mathrm{c}=0.2, \mathrm{CHCl}_{3}\right)$.

HPLC : Chiralcel OD, $10 \%$ iPrOH/Hexane, $0.50 \mathrm{~mL} / \mathrm{min}$., UV detection at $210 \mathrm{~nm}$, major peak at 15.6 min., minor peak at 12.5 min., $96 \%$ ee.

\section{(S,E)-1-(benzyloxy)-6-(trimethylsilyl)hex-4-en-2-ol (3d)}

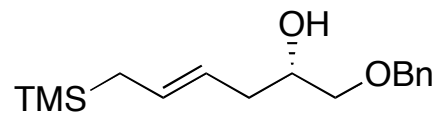

Flash chromatography (8\% EtOAc/hexanes) yielded a colourless oil (39 mg, 70\%), which gave satisfactory analytical data.

TLC (25\% EtOAc/hexanes, $\left.\mathrm{KMnO}_{4}\right)$ : Rf 0.40;

${ }^{1} \mathrm{H}$ NMR $\left(\mathrm{CDCl}_{3}, 400 \mathrm{MHz}\right): \delta$ 7.36-7.26 (m, 5H), $5.50(\mathrm{ddd}, J=15.6,8.0,8.0 \mathrm{~Hz}, 1 \mathrm{H}), 5.24(\mathrm{ddd}, J=$ 15.2, 7.1, 7.1 Hz, 1H), 4.56 (s, 2H), 3.80 (ddddd, $J=4.4,3.9,3.9,3.9,3.9 \mathrm{~Hz}, 1 \mathrm{H}), 3.52$ (dd, $J=9.5,3.4$ $\mathrm{Hz}, 1 \mathrm{H}), 3.37$ (dd, $J=9.4,7.3 \mathrm{~Hz}, 1 \mathrm{H}), 2.26(\mathrm{~d}, J=3.4 \mathrm{~Hz} 1 \mathrm{H}), 2.20(\mathrm{~d}, J=6.2,6.2 \mathrm{~Hz}, 2 \mathrm{H}), 1.45(\mathrm{~d}, J$ $=8.0 \mathrm{~Hz}, 2 \mathrm{H}),-0.01(\mathrm{~s}, 9 \mathrm{H})$;

${ }^{13} \mathrm{C} \mathrm{NMR}\left(\mathrm{CDCl}_{3}, 100 \mathrm{MHz}\right): \delta 138.1,130.1,128.4,127.7,127.7,123.5,74.0,73.4,70.3,37.0,22.9$, 2.0 ;

IR (neat): $\mathrm{cm}^{-1} 3455,3029,2953,2900,1454,1248$;

HRMS (ESP) calcd for $\mathrm{C}_{16} \mathrm{H}_{26} \mathrm{O}_{2} \mathrm{SiNa}$ : 301.1594; found: 301.1594 .

$[\alpha]^{25}-1.00\left(\mathrm{c}=1.10, \mathrm{CHCl}_{3}\right)$.

HPLC : Chiralcel OD, $5 \% \mathrm{iPrOH} / \mathrm{hexane}, 0.50 \mathrm{~mL} / \mathrm{min}$., UV detection at $210 \mathrm{~nm}$, major peak at 15.5 min., minor peak at 17.7 min., $91 \%$ ee.

\section{(S,E)-5-(trimethylsilyl)-1-phenylpent-3-en-1-ol (3e)}

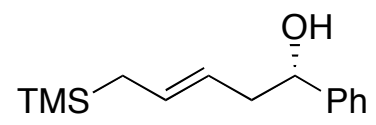

Flash chromatography (5\% EtOAc/hexanes) yielded a colorless oil (34 mg, 72\%), which gave satisfactory analytical data. 
TLC (25\% EtOAc/Hexane, $\left.\mathrm{KMnO}_{4}\right)$ : Rf 0.40;

${ }^{1} \mathrm{H}$ NMR $\left(\mathrm{CDCl}_{3}, 400 \mathrm{MHz}\right): \delta$ 7.37-7.24 (m, 5H), $5.56(\mathrm{ddd}, J=15.7,8.1,8.1 \mathrm{~Hz}, 1 \mathrm{H})$, $5.24(\mathrm{ddd}, J=15.3,7.2,7.2 \mathrm{~Hz}, 1 \mathrm{H}), 4.68(\mathrm{ddd}, J=8.0,4.9,3.2 \mathrm{~Hz}, 1 \mathrm{H}), 2.54-2.38(\mathrm{~m}, 2 \mathrm{H}), 2.06(\mathrm{~d}, J=$ $3.3 \mathrm{~Hz} 1 \mathrm{H}), 1.48(\mathrm{~d}, J=8.4 \mathrm{~Hz}, 1 \mathrm{H}),-0.02(\mathrm{~s}, 9 \mathrm{H})$;

${ }^{13} \mathrm{C} \mathrm{NMR}\left(\mathrm{CDCl}_{3}, 100 \mathrm{MHz}\right): \delta 144.0,131.3,128.3,127.3,125.8,123.6,73.5,43.0,23.1,-2.0$;

IR (neat): $\mathrm{cm}^{-1} 3363,3028,2953,2899,1658,1247$;

HRMS (ESP) calcd for $\mathrm{C}_{14} \mathrm{H}_{22} \mathrm{OSiNa}$ : 257.1332; found: 257.1332 .

$[\alpha]_{D}^{25}-23.15\left(\mathrm{c}=1.1, \mathrm{CHCl}_{3}\right)$.

HPLC : Chiralcel OD, $10 \%$ PrOH/Hexane, $0.50 \mathrm{~mL} / \mathrm{min}$., $\mathrm{UV}$ detection at $210 \mathrm{~nm}$, , major peak at 9.8

min., minor peak at $9.1 \mathrm{~min} ., 95 \%$ ee.

\section{$(S, 1 E, 5 E)$-7-(trimethylsilyl)-1-phenylhepta-1,5-dien-3-ol (3f)}

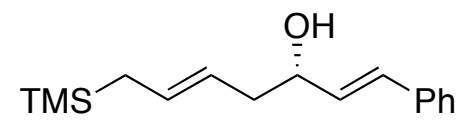

Flash chromatography (5\% EtOAc/hexanes) yielded a colorless oil (39 mg, 75\%), which gave satisfactory analytical data.

TLC (25\% EtOAc/hexanes, $\left.\mathrm{KMnO}_{4}\right)$ : Rf 0.25;

${ }^{1} \mathrm{H}$ NMR $\left(\mathrm{CDCl}_{3}, 400 \mathrm{MHz}\right): \delta 7.40-7.22(\mathrm{~m}, 5 \mathrm{H}), 6.60(\mathrm{~d}, J=16.0 \mathrm{~Hz}, 1 \mathrm{H})$, $6.25(\mathrm{dd}, J=16.0,6.2 \mathrm{~Hz} 1 \mathrm{H}), 5.58(\mathrm{ddd}, J=15.8,8.1,8.1 \mathrm{~Hz}, 1 \mathrm{H}), 5.28(\mathrm{ddd}, J=15.1,7.7,7.7 \mathrm{~Hz}$, $1 \mathrm{H}), 4.32-4.24(\mathrm{~m}, 1 \mathrm{H}), 2.44-2.26(\mathrm{~m}, 2 \mathrm{H}), 1.8(\mathrm{~d}, J=4.0 \mathrm{~Hz}, 2 \mathrm{H}), 1.50(\mathrm{~d}, J=8.1 \mathrm{~Hz}, 2 \mathrm{H}), 0.00(\mathrm{~s}$, 9H);

${ }^{13} \mathrm{C} \mathrm{NMR}\left(\mathrm{CDCl}_{3}, 100 \mathrm{MHz}\right): \delta 136.9,131.9,131.3,130.1,128.5,127.5,126.4,123.2$, 72.0, 41.2, 23.1, -2.0;

IR (neat): $\mathrm{cm}^{-1} 3398,3027,2953,2899,1678,1626,1450,1248$;

HRMS (ESP) calcd for $\mathrm{C}_{16} \mathrm{H}_{24} \mathrm{OSiNa}$ : 283.1490; found 283.1486.

HPLC : Chiralcel OD, 10\% $\mathrm{iPrOH} / \mathrm{Hexane}, 0.5 \mathrm{~mL} / \mathrm{min}$., UV detection at $210 \mathrm{~nm}$, major peak at 18.6 min., minor peak at 12.4 min., $79 \%$ ee.

\section{$(S, 2 E, 6 E)-8$-(trimethylsilyl)octa-2,6-dien-4-ol (3g)}

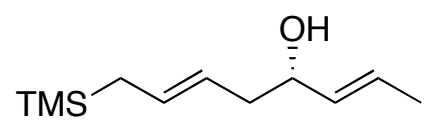

Flash chromatography (5\% EtOAc/hexanes) yielded a colorless oil (32 mg, 81\%), which gave satisfactory analytical data.

TLC (25\% EtOAc/hexanes, $\left.\mathrm{KMnO}_{4}\right):$ Rf 0.40;

${ }^{1} \mathrm{H}$ NMR $\left(\mathrm{CDCl}_{3}, 400 \mathrm{MHz}\right): \delta 5.68$ (ddddd, $\left.J=15.2,6.5,6.5,6.5,1.0 \mathrm{~Hz}, 1 \mathrm{H}\right), 5.56-5.46(\mathrm{~m}, 2 \mathrm{H}), 5.24$ (ddd, $J=15.1,7.2,7.2 \mathrm{~Hz}, 1 \mathrm{H}), 4.05$ (ddd, $J=11.5,6.3,6.3 \mathrm{~Hz}, 1 \mathrm{H}), 3.32-3.27(\mathrm{~m}, 1 \mathrm{H}), 2.30-2.16$ (m, 2H), 1.70 (ddd, $J=6.4,1.5,0.7 \mathrm{~Hz}, 3 \mathrm{H}), 1.62(\mathrm{~d}, J=3.7 \mathrm{~Hz}, 1 \mathrm{H}), 1.47$ (d, $J=8.1 \mathrm{~Hz}, 2 \mathrm{H}), 0.00(\mathrm{~s}, 9 \mathrm{H})$; ${ }^{13} \mathrm{C}$ NMR $\left(\mathrm{CDCl}_{3}, 100 \mathrm{MHz}\right): \delta$ 133.5, 130.7, 126.7, 123.6, 72.1, 41.1, 23.0, 17.7, -2.0;

IR (neat): $\mathrm{cm}^{-1} 3344,2955,2917,1439,1248$;

HRMS (ESP) calcd for $\mathrm{C}_{11} \mathrm{H}_{22}$ OSiNa: 221.1332; found: 221.1333 . $[\alpha]_{D}^{25}-4.77\left(\mathrm{c}=0.90, \mathrm{CHCl}_{3}\right)$. 
HPLC : Chiralcel OD, $1 \%$ PrOH/Hexane, $0.5 \mathrm{~mL} / \mathrm{min}$., UV detection at $210 \mathrm{~nm}$, major peak at 19.2 min., minor peak at $24.5 \mathrm{~min} ., 96 \%$ ee.

\section{$(S, E)-1-(t r i m e t h y l s i l y l) d o d e c-2-e n-6-y n-5-o l ~(3 h)$}

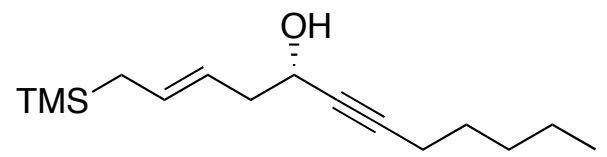

Flash chromatography (5\% EtOAc/Toluene) yielded a colorless oil (43 mg, 85\%), which gave satisfactory analytical data.

TLC (25\% EtOAc/Hexane, $\left.\mathrm{KMnO}_{4}\right)$ : Rf 0.50;

${ }^{1} \mathrm{H}$ NMR $\left(\mathrm{CDCl}_{3}, 400 \mathrm{MHz}\right): \delta 5.56(\mathrm{ddd}, J=15.6,8.1,8.1 \mathrm{~Hz}, 1 \mathrm{H})$, 5.30 (ddd, $J=15.1,7.3,7.3 \mathrm{~Hz}, 1 \mathrm{H}), 4.32$ (ddddd, $J=6.1,6.1,6.1,1.8,1.8 \mathrm{~Hz}, 1 \mathrm{H}), 2.38(\mathrm{dd}, J=7.0$, $7.0 \mathrm{~Hz} 2 \mathrm{H}), 2.20$ (dddd, $J=7.0,7.0,1.9,1.9 \mathrm{~Hz} 2 \mathrm{H}), 1.82(\mathrm{~d}, J=6.1 \mathrm{~Hz} 1 \mathrm{H}), 1.52(\mathrm{~m}, 2 \mathrm{H}), 1.48(\mathrm{~d}, J=$ $7.5 \mathrm{~Hz}, 2 \mathrm{H}), 1.40-1.28(\mathrm{~m}, 4 \mathrm{H}), 0.90(\mathrm{dd}, J=6.9 \mathrm{~Hz}, 3 \mathrm{H}), 0.01(\mathrm{~s}, 9 \mathrm{H})$;

${ }^{13} \mathrm{C} \mathrm{NMR}\left(\mathrm{CDCl}_{3}, 100 \mathrm{MHz}\right): \delta 131.4,122.6,85.6,80.9,62.3,41.6,31.0,28.4,23.1,22.2,18.7,14.0$, 2.0 ;

IR (neat): $\mathrm{cm}^{-1} 3352,2955,2933,2861,2226,1248$;

HRMS (ESP) calcd for $\mathrm{C}_{15} \mathrm{H}_{28} \mathrm{OSiNa}$ : 275.1802; found: 275.1804 .

$[\alpha]_{D}^{25}-8.70\left(\mathrm{c}=1.20, \mathrm{CHCl}_{3}\right)$.

HPLC : Chiralcel OD, $2.5 \% \mathrm{iPrOH} /$ hexane, $0.50 \mathrm{~mL} / \mathrm{min}$., UV detection at $210 \mathrm{~nm}$, major peak at 8.4 min., minor peak at 16.6 min., $95 \%$ ee.

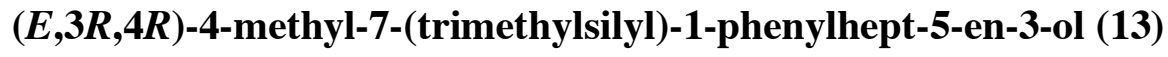

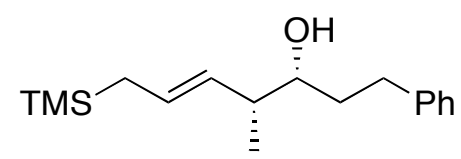

Flash chromatography (5\% EtOAc/toluene) yielded a colorless oil (47 mg, 85\%).

TLC (25\% EtOAc/hexanes, $\left.\mathrm{KMnO}_{4}\right):$ Rf 0.50;

${ }^{1} \mathrm{H}$ NMR $\left(\mathrm{CDCl}_{3}, 400 \mathrm{MHz}\right): \delta$ 7.32-7.18 (m, 5H), $5.50(\mathrm{ddd}, J=15.6,8.1,8.1 \mathrm{~Hz}, 1 \mathrm{H}), 5.15$ (dddd, $J=$ 15.2, 7.9, 0.9, $0.9 \mathrm{~Hz}, 1 \mathrm{H}), 4.55$ (dddd, $J=11.1,5.6,5.6,3.0 \mathrm{~Hz} 1 \mathrm{H}), 2.90$ (ddd, $J=13.8,10.3,5.1 \mathrm{~Hz}$ $1 \mathrm{H}), 2.65$ (ddd, $J=13.8,9.9,6.6 \mathrm{~Hz} 1 \mathrm{H}), 2.26$ (dddd, $J=14.1,6.9,6.9,6.9 \mathrm{~Hz} 1 \mathrm{H}), 1.90-1.80$ (m, $1 \mathrm{H}$ ), $1.70-1.60(\mathrm{~m}, 1 \mathrm{H}), 1.46-1.38(\mathrm{~m}, 3 \mathrm{H}), 1.01(\mathrm{~d}, J=6.9 \mathrm{~Hz}, 3 \mathrm{H}), 0.00(\mathrm{~s}, 9 \mathrm{H})$;

${ }^{13} \mathrm{C} \mathrm{NMR}\left(\mathrm{CDCl}_{3}, 100 \mathrm{MHz}\right): \delta 142.4,130.4,128.5,128.3,127.9,125.7,74.6,43.1$, $35.8,32.5,22.9,15.6,-2.0$

IR (neat): $\mathrm{cm}^{-1} 3375,3028,2955,1454,1248$;

HRMS (ESP) calcd for $\mathrm{C}_{17} \mathrm{H}_{29} \mathrm{OSi}$ : 277.1982; found: 277.1984 .

$[\alpha]_{\mathrm{D}}^{25}+14.10\left(\mathrm{c}=0.50, \mathrm{CHCl}_{3}\right)$.

HPLC : Chiralcel OD, $10 \%$ PrOH/Hexane, $0.50 \mathrm{~mL} / \mathrm{min}$., UV detection at $254 \mathrm{~nm}$, Major peak at 12.4 min., minor peak at 9.5 min., $94 \%$ ee.

\section{(E,2S,3R)-1-(benzyloxy)-3-methyl-6-(trimethylsilyl)hex-4-en-2-ol (14)}




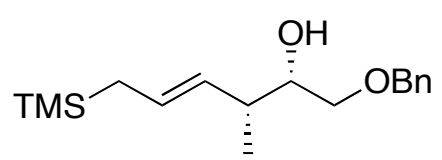

Flash chromatography (10\% EtOAc/hexanes) yielded a colorless oil (53 mg, 90\%).

TLC (15\% EtOAc/Hexane, $\left.\mathrm{KMnO}_{4}\right)$ : Rf 0.30;

${ }^{1} \mathrm{H}$ NMR $\left(\mathrm{CDCl}_{3}, 400 \mathrm{MHz}\right): \delta$ 7.36-7.26 (m, 5H), 5.45 (ddd, $\left.J=15.7,8.1,8.1 \mathrm{~Hz}, 1 \mathrm{H}\right), 5.15$ (dddd, $J=$ $15.2,8.3,1.3,1.3 \mathrm{~Hz}, 1 \mathrm{H}), 4.55(\mathrm{~s}, 2 \mathrm{H}), 3.60-3.55(\mathrm{~m}, 1 \mathrm{H}), 3.37(\mathrm{dd}, J=10.0,8.4 \mathrm{~Hz}, 1 \mathrm{H}), 2.34(\mathrm{~d}, J=$ $3.6 \mathrm{~Hz}, 1 \mathrm{H}), 2.25$ (dddd, $J=14.6,7.4,7.4,7.4 \mathrm{~Hz} 1 \mathrm{H}), 1.40(\mathrm{dd}, J=8.1,1.2 \mathrm{~Hz}, 2 \mathrm{H}), 1.06(\mathrm{~d}, J=6.8$ $\mathrm{Hz}, 3 \mathrm{H}),-0.02(\mathrm{~s}, 9 \mathrm{H})$;

${ }^{13} \mathrm{C} \mathrm{NMR}\left(\mathrm{CDCl}_{3}, 100 \mathrm{MHz}\right): \delta 138.1,130.2,128.4,127.7,127.7,127.2,74.0,73.4$, 73.1, 40.6, 22.8, 17.0, -2.0;

IR (neat): $\mathrm{cm}^{-1} 3466,2956,2900,2870,1454,1248$;

HRMS (ESP) calcd for $\mathrm{C}_{17} \mathrm{H}_{28} \mathrm{O}_{2} \mathrm{SiNa}$ : 315.1751; found: 315.1753 .

$[\alpha]_{D}^{25}+22.83\left(\mathrm{c}=0.30, \mathrm{CHCl}_{3}\right)$.

HPLC : Chiralcel OD, $10 \%$ PrOH/Hexane, $0.50 \mathrm{~mL} / \mathrm{min}$., UV detection at $210 \mathrm{~nm}$, Major peak at 10.0 min., minor peak at 11.1 min., $96 \%$ ee.

\section{$(E, 1 S, 2 R)-2-m e t h y l-5-(t r i m e t h y l s i l y l)-1-p h e n y l p e n t-3-e n-1-o l)(15)$}

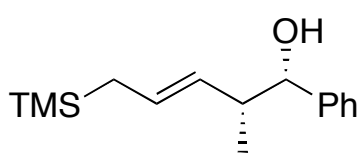

Flash chromatography (5\% EtOAc/hexanes) yielded a colorless oil (47 mg, 94\%), which gave satisfactory analytical data.

TLC (25\% EtOAc/hexanes, $\left.\mathrm{KMnO}_{4}\right)$ : Rf 0.50;

${ }^{1} \mathrm{H} \mathrm{NMR}\left(\mathrm{CDCl}_{3}, 400 \mathrm{MHz}\right): \delta$ 7.36-7.22 (m, 5H), 5.45 (ddd, $\left.J=15.6,8.1,8.1 \mathrm{~Hz}, 1 \mathrm{H}\right), 5.15$ (dddd, $J=$ $15.2,10.5,1.3,1.3 \mathrm{~Hz}, 1 \mathrm{H}), 4.55(\mathrm{dd}, J=5.6,4.0 \mathrm{~Hz} 1 \mathrm{H}), 2.56$ (dddd, $J=13.2,6.9,6.9,6.9 \mathrm{~Hz} 1 \mathrm{H})$, $1.98(\mathrm{~m}, 1 \mathrm{H}), 1.44(\mathrm{~d}, J=8.0 \mathrm{~Hz}, 2 \mathrm{H}), 0.98(\mathrm{~d}, J=6.9 \mathrm{~Hz}, 3 \mathrm{H}),-0.05(\mathrm{~s}, 9 \mathrm{H})$;

${ }^{13} \mathrm{C} \mathrm{NMR}\left(\mathrm{CDCl}_{3}, 100 \mathrm{MHz}\right): \delta 142.8,129.9,128.1,128.0,127.2,126.6,77.7,44.0$, $22.9,15.4,-2.0$;

IR (neat): $\mathrm{cm}^{-1} 3393,2956,2875,1453,1248$;

HRMS (ESP) calcd for $\mathrm{C}_{15} \mathrm{H}_{24}$ OSiNa: 271.1489; found: 271.1487 .

$[\alpha]^{25}-12.15\left(\mathrm{c}=0.6, \mathrm{CHCl}_{3}\right)$.

HPLC : Chiralcel OD, 2.5\% $i \mathrm{PrOH} / \mathrm{Hexane}, 0.5 \mathrm{~mL} / \mathrm{min}$., UV detection at $254 \mathrm{~nm}$, major peak at 15.2 min., minor peak at 13.7 min., $93 \%$ ee.

\section{$(E, 3 R, 4 S)-4-m e t h y l-7-(t r i m e t h y l s i l y l)-1-p h e n y l h e p t-5-e n-3-o l ~(16)$}

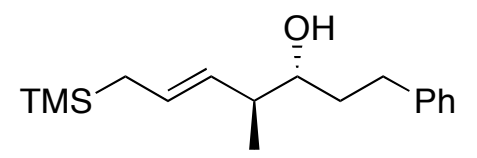


Flash chromatography (10\% EtOAc/hexanes) yielded a colorless oil (47 mg, 85\%).

TLC (25\% EtOAc/Hexane, $\left.\mathrm{KMnO}_{4}\right)$ : Rf 0.50;

${ }^{1} \mathrm{H}$ NMR $\left(\mathrm{CDCl}_{3}, 400 \mathrm{MHz}\right): \delta$ 7.32-7.18 (m, 5H), $5.50(\mathrm{ddd}, J=15.8,8.1,8.1 \mathrm{~Hz}, 1 \mathrm{H}), 5.15$ (dddd, $J=$ $15.3,8.6,1.2,1.2 \mathrm{~Hz}, 1 \mathrm{H}), 3.38-3.30(\mathrm{~m}, 1 \mathrm{H}), 2.86(\mathrm{ddd}, J=13.8,10.5,5.1 \mathrm{~Hz} 1 \mathrm{H}), 2.65(\mathrm{ddd}, J=13.7$, $10,0,6.4 \mathrm{~Hz} 1 \mathrm{H}), 2.15$ (dddd, $J=13.4,6.4,6.4,6.4 \mathrm{~Hz} 1 \mathrm{H}), 1.90-1.80(\mathrm{~m}, 1 \mathrm{H}), 1.74-1.64(\mathrm{~m}, 1 \mathrm{H}), 1.71$ $(\mathrm{d}, J=3.7 \mathrm{~Hz}, 1 \mathrm{H}), 1.47(\mathrm{dd}, J=8.0,1.2 \mathrm{~Hz}, 2 \mathrm{H}), 1.01(\mathrm{~d}, J=6.9 \mathrm{~Hz}, 3 \mathrm{H}), 0.01(\mathrm{~s}, 9 \mathrm{H})$;

${ }^{13} \mathrm{C} \mathrm{NMR}\left(\mathrm{CDCl}_{3}, 100 \mathrm{MHz}\right): \delta 1142.5,130.1,129.2,128.5,128.3,125.7,74.3,43.8$,

$36.1,32.2,23.0,17.1,-2.0$;

IR (neat): $\mathrm{cm}^{-1} 3410,2955,2874,1454,1248$;

HRMS (ESP) calcd for $\mathrm{C}_{17} \mathrm{H}_{28} \mathrm{OSiNa}$ : 299.1802; found 299.1803.

$[\alpha]_{D}^{25}-10.45\left(\mathrm{c}=0.20, \mathrm{CHCl}_{3}\right)$.

HPLC : Chiralcel OD, 10\% $i \mathrm{PrOH} / \mathrm{Hexane}, 0.50 \mathrm{~mL} / \mathrm{min}$., UV detection at $210 \mathrm{~nm}$, Major peak at 13.6 min., minor peak at $15.2 \mathrm{~min} ., 95 \%$ ee.

(E,2S,3S)-1-(benzyloxy)-3-methyl-6-(trimethylsilyl)hex-4-en-2-ol (17)

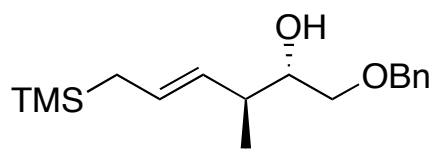

Flash chromatography (10\% EtOAc/hexanes) yielded a colourless oil (38 mg, 58\%).

TLC (25\% EtOAc/Hexane, $\left.\mathrm{KMnO}_{4}\right)$ : Rf 0.40;

${ }^{1} \mathrm{H} \mathrm{NMR}\left(\mathrm{CDCl}_{3}, 400 \mathrm{MHz}\right): \delta 7.32-7.18(\mathrm{~m}, 5 \mathrm{H}), 5.50(\mathrm{ddd}, J=15.8,8.1,8.1 \mathrm{~Hz}, 1 \mathrm{H}), 5.15(\mathrm{dddd}, J=$ $15.3,8.6,1.2,1.2 \mathrm{~Hz}, 1 \mathrm{H}), 3.38-3.30(\mathrm{~m}, 1 \mathrm{H}), 2.86(\mathrm{ddd}, J=13.8,10.5,5.1 \mathrm{~Hz} 1 \mathrm{H}), 2.65(\mathrm{ddd}, J=13.7$, $10,0,6.4 \mathrm{~Hz} 1 \mathrm{H}), 2.15$ (dddd, $J=13.4,6.4,6.4,6.4 \mathrm{~Hz} 1 \mathrm{H}), 1.90-1.80(\mathrm{~m}, 1 \mathrm{H}), 1.74-1.64(\mathrm{~m}, 1 \mathrm{H}), 1.71$ $(\mathrm{d}, J=3.7 \mathrm{~Hz}, 1 \mathrm{H}), 1.47(\mathrm{dd}, J=8.0,1.2 \mathrm{~Hz}, 2 \mathrm{H}), 1.01(\mathrm{~d}, J=6.9 \mathrm{~Hz}, 3 \mathrm{H}), 0.01(\mathrm{~s}, 9 \mathrm{H})$;

${ }^{13} \mathrm{C} \mathrm{NMR}\left(\mathrm{CDCl}_{3}, 100 \mathrm{MHz}\right): \delta 1142.5,130.1,129.2,128.5,128.3,125.7,74.3,43.8$, $36.1,32.2,23.0,17.1,-2.0$;

IR (neat): $\mathrm{cm}^{-1} 3410,2955,2874,1454,1248$;

HRMS (ESP) calcd for $\mathrm{C}_{17} \mathrm{H}_{28}$ OSiNa: 299.1802; found: 299.1803.

$[\alpha]_{D}^{25}-23.40\left(\mathrm{c}=0.5, \mathrm{CHCl}_{3}\right)$.

HPLC : Chiralcel OD, $10 \%$ PrOH/Hexane, $0.50 \mathrm{~mL} / \mathrm{min}$., UV detection at $210 \mathrm{~nm}$, Major peak at 9.5

min., minor peak at 11.3 min., $98 \%$ ee.

$(E, 1 S, 2 S)-2-m e t h y l-5-(t r i m e t h y l s i l y l)-1-p h e n y l p e n t-3-e n-1-o l)(18)$

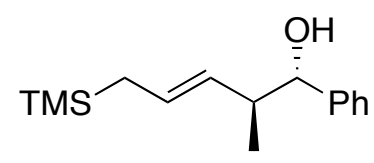

Flash chromatography (10\% EtOAc/hexanes) yielded a colorless oil (49 mg, 98\%).

TLC (25\% EtOAc/Toluene, $\left.\mathrm{KMnO}_{4}\right)$ : Rf 0.5;

${ }^{1} \mathrm{H}$ NMR $\left(\mathrm{CDCl}_{3}, 400 \mathrm{MHz}\right): \delta$ 7.36-7.26 (m, 5H), 5.45 (ddd, $\left.J=15.8,8.1,8.1 \mathrm{~Hz}, 1 \mathrm{H}\right), 5.15$ (dddd, $J=$ $15.2,8.6,1.1,1.1 \mathrm{~Hz}, 1 \mathrm{H}), 4.26(\mathrm{dd}, J=8.2,2.1 \mathrm{~Hz} 1 \mathrm{H}), 2.40(\mathrm{dddd}, J=14.8,6.9,6.9,6.9 \mathrm{~Hz} 1 \mathrm{H}), 2.27$ $(\mathrm{d}, J=2.2 \mathrm{~Hz}, 1 \mathrm{H}), 1.52(\mathrm{dd}, J=8.1,1.2 \mathrm{~Hz}, 2 \mathrm{H}), 0.84(\mathrm{~d}, J=6.9 \mathrm{~Hz}, 3 \mathrm{H}), 0.03(\mathrm{~s}, 9 \mathrm{H})$; 
${ }^{13} \mathrm{C} \mathrm{NMR}\left(\mathrm{CDCl}_{3}, 100 \mathrm{MHz}\right): \delta$ 142.6, 130.4, 130.0, 128.1, 127.5, 127.0, 78.0, 46.0, 23.1, 17.4, -2.0;

IR (neat): $\mathrm{cm}^{-1} 3430,3030,2956,2895,1453,1248$;

HRMS (ESP) calcd for $\mathrm{C}_{15} \mathrm{H}_{24}$ OSiNa: 271.1489; found: 271.1490.

$[\alpha]_{D}^{25}-183.28\left(\mathrm{c}=0.4, \mathrm{CHCl}_{3}\right)$.

HPLC : Chiralcel OD, $10 \% \mathrm{iPrOH} / \mathrm{Hexane}, 0.50 \mathrm{~mL} / \mathrm{min}$., UV detection at $210 \mathrm{~nm}$, Major peak at 10.7

min., minor peak at $8.5 \mathrm{~min} ., 95 \%$ ee.

\section{Determination of absolute stereochemistry of compound 18.}

Compound 18 was transformed to the known 2-methyl-1-phenylpropane-1,3-diol, ${ }^{1}$ which has $[\alpha]^{25}{ }_{D}^{-}$ $29.89\left(\mathrm{c}=1.5, \mathrm{CHCl}_{3}\right)$. Based on the comparison of the sign of optical rotation reported in the literature

${ }^{1}$ to the one obtained from compound $\mathbf{1 8}$, the absolute stereochemistry is as follow.<smiles>C[C@@H](CO)[C@@H](O)c1ccccc1</smiles>

(1S,2S)-2-methyl-1-phenylpropane-1,3-diol

\section{General procedure for the synthesis of compounds 5-8, 21, 22.}

At $-78{ }^{\circ} \mathrm{C}$, to a flame-dried round bottom flask, was added $3(0.2 \mathrm{mmol})$, aldehyde $(0.2 \mathrm{mmol})$ and $2 \mathrm{~mL}$ of dichloromethane. The mixture was stirred for $5 \mathrm{~min}$, and then TMSOTf $(0.1 \mathrm{mmol})$ was added. The reaction mixture was stirred further $4 \mathrm{~h}$ at $-78{ }^{\circ} \mathrm{C}$ and then quenched with an aqueous $\mathrm{NaHCO}_{3}$ solution, extracted with ether, dried over anhydrous $\mathrm{Na}_{2} \mathrm{SO}_{4}$, and filtered. The products were purified by flash chromatography. The relative stereochemistry was assigned based on TROESY NMR technique.

tetrahydro-5-phenethyl-2-phenyl-3-vinylfuran (5)

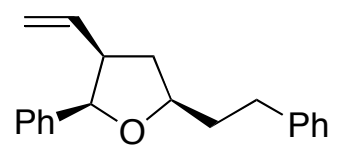

Flash chromatography (5\% EtOAc/Hexane) gave the pure product as a colourless oil (75\% yield), which gave satisfactory analytical data.

${ }^{1} \mathrm{H} \mathrm{NMR}\left(\mathrm{CDCl}_{3}, 400 \mathrm{MHz}\right): \delta$ 7.35-7.20 (m, 10H), $5.20(\mathrm{ddd}, J=17.0,10.0,8.7 \mathrm{~Hz}, 1 \mathrm{H}), 5.06(\mathrm{~d}, J=$ $8.1 \mathrm{~Hz}, 1 \mathrm{H}), 4.90(\mathrm{ddd}, J=17.1,1.9,1.0 \mathrm{~Hz}, 1 \mathrm{H}), 4.78(\mathrm{dd}, J=10.0,1.8 \mathrm{~Hz}, 1 \mathrm{H}), 4.56(\mathrm{dddd}, J=11.1$, 7.3, 5.5, $5.5 \mathrm{~Hz} 1 \mathrm{H}$ ), 3.2 (dddd, $J=8.3,8.3,8.3,8.3 \mathrm{~Hz} 1 \mathrm{H}), 2.92-2.74(\mathrm{~m}, 2 \mathrm{H}), 2.25-2.15(\mathrm{~m}, 2 \mathrm{H})$, 2.05-1.97 (m, 1H), 1.60 (ddd, $J=12.3,9.2,9.2 \mathrm{~Hz}, 1 \mathrm{H})$;

${ }^{13} \mathrm{C} \mathrm{NMR}\left(\mathrm{CDCl}_{3}, 100 \mathrm{MHz}\right): \delta 142.0,140.4,138.6,128.4,128.4,127.8,127.0,126.8,125.8,114.9$, 83.2, 78.7, 48.9, 38.3, 37.3, 32.7;

IR (neat): $\mathrm{cm}^{-1} 3027,2927,2861,1495,1453,1053$;

HRMS (EI) calcd for $\mathrm{C}_{20} \mathrm{H}_{22} \mathrm{O}: 278.1671$; found: 278.1666 .

HPLC : Chiralcel OD, $10 \%$ PrOH/Hexane, $0.50 \mathrm{~mL} / \mathrm{min}$., UV detection at $210 \mathrm{~nm}$, major peak at 19.2 min., minor peak at 14.0 min., $93 \%$ ee. 
“One-pot" three-component reaction to form 5: At $-78{ }^{\circ} \mathrm{C}$, to a flame-dried round bottom flask, was added boron reagent $1(0.2 \mathrm{mmol})$, hydrocinnamaldehyde $(0.2 \mathrm{mmol})$ and $1 \mathrm{~mL}$ of dichloromethane. The mixture was stirred for $5 \mathrm{~min}$, and then $\mathrm{BF}_{3}-\mathrm{Et}_{2} \mathrm{O}(0.2 \mathrm{mmol})$ was added. The reaction mixture was stirred further $12 \mathrm{~h}$ at $-78{ }^{\circ} \mathrm{C}$ and then benzaldehyde $(0.2 \mathrm{mmol})$ was added to the reaction mixture. The reaction mixture was stirred at $-78{ }^{\circ} \mathrm{C}$ for $2 \mathrm{~h}$ followed by $0{ }^{\circ} \mathrm{C}$ for $4 \mathrm{~h}$, then quenched with an aqeous $\mathrm{NaHCO}_{3}$ solution, extracted with ether, dried over anhydrous $\mathrm{Na}_{2} \mathrm{SO}_{4}$ and filtered. The products were purified by flash chromatography. Compound 5 was obtained with dr 6:1, 91\% ee.

\section{2-heptyl-tetrahydro-5-phenethyl-3-vinylfuran (6)}

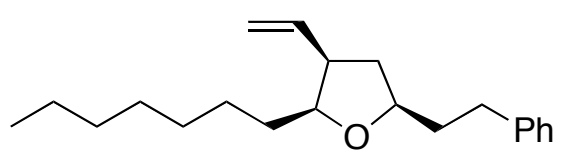

Flash chromatography (5\% EtOAc/Hexane) gave the pure product as a colourless oil (82\% yield), which gave satisfactory analytical data.

${ }^{1} \mathrm{H}$ NMR $\left(\mathrm{CDCl}_{3}, 400 \mathrm{MHz}\right): \delta$ 7.31-7.16 (m, 5H), 5.83-5.73 (m, 1H), 5.03-4.98 (m, 2H), 3.86-3.76 (m, $2 \mathrm{H}), 2.86-2.64(\mathrm{~m}, 3 \mathrm{H}), 2.20(\mathrm{ddd}, J=12.8,8,6.8 \mathrm{~Hz}, 1 \mathrm{H}), 2.03-1.94(\mathrm{~m}, 1 \mathrm{H}), 1.86-1.78(\mathrm{~m}, 1 \mathrm{H}), 1.50-$ $1.20(\mathrm{~m}, 12 \mathrm{H}), 0.92-0.86(\mathrm{~m}, 3 \mathrm{H})$;

${ }^{13} \mathrm{C} \mathrm{NMR}\left(\mathrm{CDCl}_{3}, 100 \mathrm{MHz}\right): \delta 142.2,138.8,128.4,128.3,125.7,114.9,81.7,77.8,46.8,38.2,38.2$, 32.7, 31.8, 31.8, 29.7, 29.3, 26.4, 22.7, 14.1;

IR (neat): $\mathrm{cm}^{-1} 2926,2855,1454,1050$;

HRMS (EI) calcd for $\mathrm{C}_{21} \mathrm{H}_{32} \mathrm{O}: 300.2453$; found: 300.2450 .

HPLC : Chiralcel OD, $1 \% \mathrm{iPrOH} / \mathrm{Hexane}, 0.50 \mathrm{~mL} / \mathrm{min}$., UV detection at $210 \mathrm{~nm}$, major peak at 19.2 min., minor peak at $14.0 \mathrm{~min} ., 94 \%$ ee.

\section{2-((benzyloxy)methyl)-tetrahydro-5-phenyl-3-vinylfuran (7)}

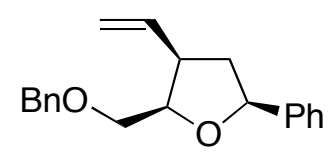

Flash chromatography (2.5\% EtOAc/Hexane) gave the pure product as a colourless oil (72\% yield), which gave satisfactory analytical data.

${ }^{1} \mathrm{H}$ NMR $\left(\mathrm{CDCl}_{3}, 400 \mathrm{MHz}\right): \delta 7.42-7.26(\mathrm{~m}, 10 \mathrm{H}), 5.85$ (ddd, $\left.J=17.1,10.0,10.0 \mathrm{~Hz}, 1 \mathrm{H}\right), 5.10$ (ddd, $J$ $=17.1,1.8,1.0 \mathrm{~Hz}, 1 \mathrm{H}), 5.05(\mathrm{ddd}, J=10.1,1.8,0.7 \mathrm{~Hz}, 1 \mathrm{H}), 4.95(\mathrm{dd}, J=9.7,6.0 \mathrm{~Hz}, 1 \mathrm{H}), 4.58(\mathrm{~d}, J=$ $1.8 \mathrm{~Hz} 1 \mathrm{H}), 4.33-4.28(\mathrm{~m}, 1 \mathrm{H}), 3.64(\mathrm{dd}, J=10.3,4.0 \mathrm{~Hz}, 1 \mathrm{H}), 3.58(\mathrm{dd},, J=10.2,5.4 \mathrm{~Hz}, 1 \mathrm{H}), 3.15$ (dddd, $J=8.7,8.7,8.7,8.7 \mathrm{~Hz} 1 \mathrm{H}), 2.40$ (ddd, $J=13.1,7.0,7.0 \mathrm{~Hz}, 1 \mathrm{H}), 1.90$ (ddd, $J=12.3,9.7,9.7$ $\mathrm{Hz}, 1 \mathrm{H})$;

${ }^{13} \mathrm{C} \mathrm{NMR}\left(\mathrm{CDCl}_{3}, 100 \mathrm{MHz}\right): \delta 142.4,138.4,136.9,128.3,128.2,127.7,127.5,127.3,126.1,116,81.0$, 80.6, 73.4, 71.2, 46.9, 41.1;

IR (neat): $\mathrm{cm}^{-1} 3065,2863,1640,1453,1090$; 
HRMS (ESP) calcd for $\mathrm{C}_{20} \mathrm{H}_{22} \mathrm{O}_{2} \mathrm{Na}$ : 317.1512; found: 317.1514.

$[\alpha]_{D}^{25}-56.13\left(\mathrm{c}=0.60, \mathrm{CHCl}_{3}\right)$.

HPLC : Chiralcel OD, 5\% $i \mathrm{PrOH} / \mathrm{Hexane,} 0.50 \mathrm{~mL} / \mathrm{min}$., UV detection at $210 \mathrm{~nm}$, major peak at 15.4 min., minor peak at 13.4 min., $91 \%$ ee.

\section{$(2 S, 3 R, 5 R)$-tetrahydro-2-methyl-5-phenethyl-2-propyl-3-vinylfuran $(8)$}

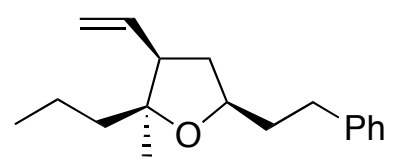

Flash chromatography (5\% EtOAc/hexanes) gave the pure product as a colourless oil with dr improving to $>25: 1$ ( $75 \%$ yield), which gave satisfactory analytical data.

${ }^{1} \mathrm{H}$ NMR $\left(\mathrm{CDCl}_{3}, 400 \mathrm{MHz}\right): \delta$ 7.31-7.16 (m, $\left.5 \mathrm{H}\right), 5.78(\mathrm{ddd}, J=18.3,10.2,8.2 \mathrm{~Hz}, 1 \mathrm{H}), 5.10-5.0(\mathrm{~m}$, $2 \mathrm{H}), 4.05-3.97(\mathrm{~m}, 1 \mathrm{H}), 2.80-2.55(\mathrm{~m}, 3 \mathrm{H}), 2.12$ (ddd, $J=12.3,5.9,5.9 \mathrm{~Hz}, 1 \mathrm{H}), 1.97-1.87(\mathrm{~m}, 1 \mathrm{H})$, $1.82-1.72(\mathrm{~m}, 1 \mathrm{H}), 1.64(\mathrm{ddd}, J=11.5,11.5,9.6 \mathrm{~Hz}, 1 \mathrm{H} 2 \mathrm{H}), 1.54-1.23$ (m, 4H), 1.22 (s, 3H), 0.91 (dd, , $J=7.1,7.1 \mathrm{~Hz}, 3 \mathrm{H})$;

${ }^{13} \mathrm{C} \mathrm{NMR}\left(\mathrm{CDCl}_{3}, 100 \mathrm{MHz}\right): \delta 142.3,137.4,128.3,128.3,125.7,115.7,83.9 .76 .9$, 55.0, 40.0, 39.0, 37.9, 32.6, 25.1, 16.9, 14.9;

IR (neat): $\mathrm{cm}^{-1} 3081,3002,2958,2871,1639,1455,1050$;

HRMS (EI) calcd for $\mathrm{C}_{18} \mathrm{H}_{26} \mathrm{O}: 258.1984$; found: 258.1979.

HPLC : Chiralcel OD, Hexane, $0.50 \mathrm{~mL} / \mathrm{min}$., UV detection at $210 \mathrm{~nm}$, major peak at $17.4 \mathrm{~min}$., minor peak at $16.0 \mathrm{~min} ., 96 \%$ ee.

\section{2-((benzyloxy)methyl)-tetrahydro-4-methyl-5-phenethyl-3-vinylfuran (21)}

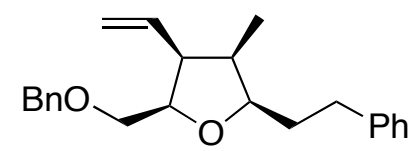

Flash chromatography (2.5\% EtOAc/Hexane) gave the pure product as a colourless oil with dr $>25: 1$ (72\% yield), which gave satisfactory analytical data.

${ }^{1} \mathrm{H}$ NMR $\left(\mathrm{CDCl}_{3}, 400 \mathrm{MHz}\right): \delta 7.40-7.18(\mathrm{~m}, 10 \mathrm{H}), 5.71(\mathrm{ddd}, J=16.3,10.6,10.6 \mathrm{~Hz}, 1 \mathrm{H}), 5.10-5.02$ $(\mathrm{m}, 2 \mathrm{H}), 4.63(\mathrm{~d}, J=12.3 \mathrm{~Hz}, 1 \mathrm{H}), 4.58(\mathrm{~d}, J=12.3 \mathrm{~Hz}, 1 \mathrm{H}), 4.12$ (dd, $J=11.4,6.0 \mathrm{~Hz} 1 \mathrm{H}), 3.95$ (ddd, $J=11.7,7.2,4.6 \mathrm{~Hz}, 1 \mathrm{H}), 3.55-3.52(\mathrm{~m}, 2 \mathrm{H}), 2.94-2.82(\mathrm{~m}, 2 \mathrm{H}), 2.65$ (ddd, $J=16.3,10.0,6.2 \mathrm{~Hz} 1 \mathrm{H})$, $2.48-2.38(\mathrm{~m}, 1 \mathrm{H}), 1.86-1.69(\mathrm{~m}, 2 \mathrm{H}), 0.87(\mathrm{~d}, J=7.4 \mathrm{~Hz}, 3 \mathrm{H})$;

${ }^{13} \mathrm{C} \mathrm{NMR}\left(\mathrm{CDCl}_{3}, 100 \mathrm{MHz}\right): \delta 142.4,138.5,134.9,128.4,128.3,128.3,127.7,125.7,117.6,80.5$, 79.6, 73.3, 71.1, 51.1, 39.9, 34.0, 33.1, 10.9;

IR (neat): $\mathrm{cm}^{-1} 2919,2858,1602,1453,1085$;

HRMS (ESP) calcd for $\mathrm{C}_{23} \mathrm{H}_{28} \mathrm{O}_{2} \mathrm{Na}$ : 359.1982; found: 359.1978.

$[\alpha]_{D}^{25}+3.95\left(\mathrm{c}=0.50, \mathrm{CHCl}_{3}\right)$.

HPLC : Chiralcel OD, Hexane, $1.00 \mathrm{~mL} / \mathrm{min}$., UV detection at $210 \mathrm{~nm}$, major peak at $5.5 \mathrm{~min}$., minor peak at $7.0 \mathrm{~min} ., 93 \%$ ee. 


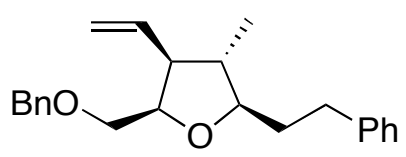

Flash chromatography (5\% EtOAc/Hexane) gave the pure product as a colourless oil with dr $>25: 1(75 \%$ yield), which gave satisfactory analytical data.

${ }^{1} \mathrm{H} \mathrm{NMR}\left(\mathrm{CDCl}_{3}, 400 \mathrm{MHz}\right): \delta 7.38-7.16(\mathrm{~m}, 10 \mathrm{H}), 5.71(\mathrm{ddd}, J=17.4,9.5,9.5 \mathrm{~Hz}, 1 \mathrm{H}), 5.10-5.02(\mathrm{~m}$, $2 \mathrm{H}), 4.58(\mathrm{~s}, 2 \mathrm{H}), 4.16(\mathrm{ddd}, J=8.9,5.2,3.9 \mathrm{~Hz} 1 \mathrm{H}), 3.53(\mathrm{dd}, J=10.3,4.0 \mathrm{~Hz}, 1 \mathrm{H}), 3.50-3.44(\mathrm{~m}$, 2H), 2.94-2.84 (m, 1H), 2.76-2.68 (m, 1H), $2.50(\mathrm{dd}, J=9.3,9.3 \mathrm{~Hz}, 1 \mathrm{H}), 1.98-1.69(\mathrm{~m}, 3 \mathrm{H}), 0.93(\mathrm{~d}, J$ $=6.6 \mathrm{~Hz}, 3 \mathrm{H})$;

${ }^{13} \mathrm{C} \mathrm{NMR}\left(\mathrm{CDCl}_{3}, 100 \mathrm{MHz}\right): \delta 142.5,136.6,128.4,128.3,127.6,127.4,125.7,116.9,85.3,79.5,73.3$, 71.3, 55.2, 44.2, 36.1, 32.6, 15.1;

IR (neat): $\mathrm{cm}^{-1} 2919,2858,1602,1453,1085$;

HRMS (ESP) calcd for $\mathrm{C}_{23} \mathrm{H}_{28} \mathrm{O}_{2} \mathrm{Na}$ : 359.1982; found: 359.1986.

$[\alpha]_{D}^{25}-2.76\left(\mathrm{c}=0.70, \mathrm{CHCl}_{3}\right)$.

HPLC : Chiralcel OD, Hexane, $1.00 \mathrm{~mL} / \mathrm{min}$., UV detection at $210 \mathrm{~nm}$, major peak at 5.2 min., minor peak at $6.6 \mathrm{~min} ., 95 \%$ ee.

\section{General procedure for the synthesis of compounds $9-11 .^{4}$}

At $-78{ }^{\circ} \mathrm{C}$, to a flame-dried round bottom flask, was added $3(0.2 \mathrm{mmol}), 2,6$-lutidine $(0.2 \mathrm{mmol})$ and 2 $\mathrm{mL}$ of dichloromethane. The mixture was stirred for $2 \mathrm{~min}$, and then $\operatorname{Tf}_{2} \mathrm{O}(0.2 \mathrm{mmol})$ was added followed by Hunig's base $(1 \mathrm{mmol})$. The reaction mixture was slowly warmed up to room temperature. After removing the solvent, the product wwas purified by flash chromatography. The relative stereochemistry for cyclpropane was assigned based on coupling constants and NOE NMR experiments. ${ }^{3}$

\section{1-(2-((1S,2R)-2-vinylcyclopropyl)ethyl)benzene (9)}

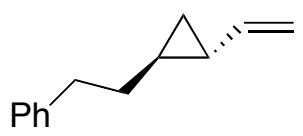

Flash chromatography (Hexane) yielded a colourless oil (81\% yield), which gave satisfactory analytical data.

TLC (25\% EtOAc/toluene, $\left.\mathrm{KMnO}_{4}\right)$ : Rf 0.90;

${ }^{1} \mathrm{H}$ NMR $\left(\mathrm{CDCl}_{3}, 400 \mathrm{MHz}\right): \delta$ 7.32-7.17 (m, 5H), $5.40(\mathrm{ddd}, J=17.1,10.2,8.7 \mathrm{~Hz}, 1 \mathrm{H}), 5.02(\mathrm{dd}, J=$ $17.1,1.76 \mathrm{~Hz}, 1 \mathrm{H}), 4.85(\mathrm{dd}, J=10.2,1.8 \mathrm{~Hz}, 1 \mathrm{H}), 2.72(\mathrm{dd}, J=7.6,7.6 \mathrm{~Hz}, 1 \mathrm{H}), 1.64-1.56(\mathrm{~m}, 2 \mathrm{H})$, 1.2 (dddd, $J=8.7,8.7,4.5,4.5 \mathrm{~Hz}, 1 \mathrm{H}), 0.85-0.75(\mathrm{~m}, 1 \mathrm{H}), 0.58$ (ddd, $J=8.3,4.7,4.7 \mathrm{~Hz}, 1 \mathrm{H}), 0.54$ (ddd, $J=8.3,5.6,4.7 \mathrm{~Hz}, 1 \mathrm{H}$ );

${ }^{13} \mathrm{C} \mathrm{NMR}\left(\mathrm{CDCl}_{3}, 100 \mathrm{MHz}\right): \delta 142.3,141.9,128.5,128.2,125.7,111.2,35.8,35.7,22.6,20.7,14.0$;

IR (neat): $\mathrm{cm}^{-1}$ 2954, 1610, 1246;

HRMS (EI) calcd for $\mathrm{C}_{13} \mathrm{H}_{14}: 170.1096$; found: $170.1098 .\left[\mathrm{M}^{+}\right]-2 \mathrm{H}$

$[\alpha]_{D}^{25}+27.09\left(\mathrm{c}=0.2, \mathrm{CHCl}_{3}\right)$. 
HPLC : Chiralcel OD, 10\% $i \mathrm{PrOH} /$ hexane, $1.00 \mathrm{~mL} / \mathrm{min}$., UV detection at $254 \mathrm{~nm}$, major peak at 6.7 min., minor peak at 7.82 min., $93 \%$ ee.

\section{(1S,2R)-1-heptyl-2-vinylcyclopropane (10)}

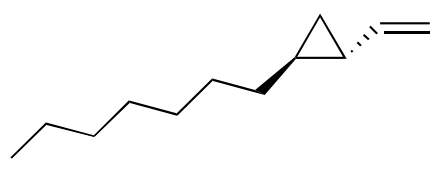

Flash chromatography (Hexane) yielded a colourless oil (72\% yield), which gave satisfactory analytical data.

TLC (25\% EtOAc/Hexane, $\left.\mathrm{KMnO}_{4}\right)$ : Rf 0.90;

${ }^{1} \mathrm{H}$ NMR $\left(\mathrm{CDCl}_{3}, 400 \mathrm{MHz}\right): \delta 5.40(\mathrm{ddd}, J=17.1,10.2,8.8 \mathrm{~Hz}, 1 \mathrm{H}), 5.05(\mathrm{dd}, J=17.1,1.8 \mathrm{~Hz} 1 \mathrm{H})$, 4.85 (dd, $J=10.2,1.8 \mathrm{~Hz} 1 \mathrm{H}), 1.40-1.24$ (m, 12H), 1.12 (dddd, $J=8.6,8.6,4.3,4.3 \mathrm{~Hz}, 1 \mathrm{H}), 0.91$ (dd, $J$ $=6.7,6.7 \mathrm{~Hz}, 3 \mathrm{H}), 0.78-0.70(\mathrm{~m}, 1 \mathrm{H}), 0.55(\mathrm{ddd}, J=8.3,4.3,4.3 \mathrm{~Hz}, 1 \mathrm{H}), 0.55(\mathrm{ddd}, J=8.2,5.5,4.5$ $\mathrm{Hz}, 1 \mathrm{H})$;

${ }^{13} \mathrm{C} \mathrm{NMR}\left(\mathrm{CDCl}_{3}, 100 \mathrm{MHz}\right): \delta$ 142.3, 110.8, 33.8, 31.9, 29.4, 29.4, 29.3, 22.7, 22.5, 21.1, 14.1, 13.9; IR (neat): $\mathrm{cm}^{-1} 2925,2854,1636,1026$;

HRMS (EI) calcd for $\mathrm{C}_{12} \mathrm{H}_{22}: 160.1722$; found: $160.1718 .\left[\mathrm{M}^{+}\right]$

HPLC : Chiralcel OD, $2.5 \% \mathrm{iPrOH} /$ hexane, $1.00 \mathrm{~mL} / \mathrm{min}$., UV detection at $254 \mathrm{~nm}$, major peak at 10.6 min., minor peak at $12.1 \mathrm{~min} ., 95 \%$ ee.

\section{1-((((1S,2R)-2-vinylcyclopropyl)methoxy)methyl)benzene (11)}

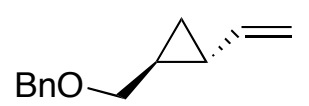

At room temperature, to a flame-dried round bottom flask, was added 3 (0.2 mmol), 2,6-lutidine (0.2 $\mathrm{mmol})$ and $2 \mathrm{~mL}$ of dichloromethane. The mixture was stirred for $1 \mathrm{~min}$, and then $\mathrm{SOCl}_{2}(0.2 \mathrm{mmol})$ was added slowly. The reaction mixture was stirred at room temperature for $4 \mathrm{~h}$. After removing the solvent, the product was purified by flash chromatography. Flash chromatography $(2.5 \%$ EtOAc/Hexane) yielded a colourless oil (56\% yield), which gave satisfactory analytical data.

TLC (25\% EtOAc/Hexane, $\left.\mathrm{KMnO}_{4}\right):$ Rf 0.80;

${ }^{1} \mathrm{H} \mathrm{NMR}\left(\mathrm{CDCl}_{3}, 400 \mathrm{MHz}\right): \delta 7.35-7.26(\mathrm{~m}, 5 \mathrm{H}), 5.42(\mathrm{ddd}, J=17.1,10.2,8.6 \mathrm{~Hz}, 1 \mathrm{H}), 5.08(\mathrm{dd}, J=$ 17.1, $1.7 \mathrm{~Hz} 1 \mathrm{H}), 4.88(\mathrm{dd}, J=10.3,1.3 \mathrm{~Hz} 1 \mathrm{H}), 4.54(\mathrm{~d}, J=2.5 \mathrm{~Hz}, 2 \mathrm{H}), 3.43(\mathrm{dd}, J=10.4,6.5 \mathrm{~Hz}$, $1 \mathrm{H}), 3.35$ (dd, $J=10.5,6.9 \mathrm{~Hz}, 1 \mathrm{H}), 1.37-1.30(\mathrm{~m}, 1 \mathrm{H}), 1.20(\mathrm{dddd}, J=13.8,6.7,6.7,4.2 \mathrm{~Hz}, 1 \mathrm{H}), 0.68$ (dd, $J=7.5,1.0 \mathrm{~Hz}, 2 \mathrm{H})$;

${ }^{13} \mathrm{C} \mathrm{NMR}\left(\mathrm{CDCl}_{3}, 100 \mathrm{MHz}\right): \delta 140.7,128.4,128.3,127.7,127.5,112.2,73.2,72.4,20.8,20.3,11.9$; IR (neat): $\mathrm{cm}^{-1}$ 2855, 1636, 1094, 1028;

HRMS (EI) calcd for $\mathrm{C}_{13} \mathrm{H}_{16} \mathrm{O}$ : 188.1201 ; found: $188.1206 .\left[\mathrm{M}^{+}\right]$

HPLC : Chiralcel OD, hexane, 1.00mL/min., UV detection at $220 \mathrm{~nm}$, major peak at $12.4 \mathrm{~min}$., minor peak at $8.0 \mathrm{~min} ., 90 \%$ ee. 


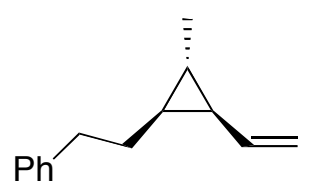

Flash chromatography (Hexane) gave the pure product as a colourless oil with $\mathrm{dr}$ 8:1 (78\% yield).

TLC (25\% EtOAc/hexane, $\left.\mathrm{KMnO}_{4}\right)$ : Rf 0.90;

${ }^{1} \mathrm{H}$ NMR (400 MHz, $\mathrm{CDCl}_{3} \delta$ 7.30-7.16 (m, 5H), 5.55 (ddd, $\left.J=17.1,10.3,9.1 \mathrm{~Hz}, 1 \mathrm{H}\right), 5.05$ (dd, $J=$ $17.1,2.1 \mathrm{~Hz} 1 \mathrm{H}), 4.95(\mathrm{dd}, J=10.3,2.1 \mathrm{~Hz} 1 \mathrm{H}), 2.70(\mathrm{ddd}, J=7.6,7.6,3 \mathrm{~Hz}, 1 \mathrm{H}), 1.60(\mathrm{dddd}, J=$ $14.3,14.3,6.7,6.7 \mathrm{~Hz} 2 \mathrm{H}), 1.22$ (dddd, $J=8.7,8.7,4.5 \mathrm{~Hz}, 1 \mathrm{H}), 1.03(\mathrm{~d},, J=6.3 \mathrm{~Hz}, 1 \mathrm{H}), 0.81-0.75$ (m, 1H), 0.56 (dddd, $J=6.8,6.8,4.7,4.7 \mathrm{~Hz}, 1 \mathrm{H})$;

${ }^{13} \mathrm{C} \mathrm{NMR}\left(\mathrm{CDCl}_{3}, 100 \mathrm{MHz}\right): \delta 142.4,138.2,128.5,128.2,125.6,113.5,35.8,35.6,27.7,27.6,20.3$, 13.6;

IR (neat): $\mathrm{cm}^{-1} 2926,1632,1453$;

HRMS (EI) calcd for $\mathrm{C}_{14} \mathrm{H}_{18}: 186.1409$; found: $186.1414 .\left[\mathrm{M}^{+}\right]$

HPLC : Chiralcel OD, $2.5 \% \mathrm{iPrOH} /$ hexane, $1.00 \mathrm{~mL} / \mathrm{min}$, UV detection at $254 \mathrm{~nm}$, major peak at 20.2

min., minor peak at 23.7 min., $94 \%$ ee.

\section{General procedure for the synthesis of compounds 19 and 20.}

At $-78{ }^{\circ} \mathrm{C}$, to a flame-dried round bottom flask, was added $1(0.2 \mathrm{mmol})$, ketoaldehyde $(0.2 \mathrm{mmol})$ and 2 $\mathrm{mL}$ of dichloromethane. The mixture was stirred for $5 \mathrm{~min}$, then $\mathrm{BF}_{3}-\mathrm{Et}_{2} \mathrm{O}(0.2 \mathrm{mmol})$ was added. The reaction mixture was stirred additional $12 \mathrm{~h}$ at $-78{ }^{\circ} \mathrm{C}$ and $2 \mathrm{~h}$ at $0{ }^{\circ} \mathrm{C}$, then quenched with an aqeous $\mathrm{NaHCO}_{3}$ solution, extracted with ether, dried over anhydrous $\mathrm{Na}_{2} \mathrm{SO}_{4}$ and filtered. The products were purified by flash chromatography. The relative stereochemistry of 19 was assigned based on TROESY NMR technique. Structure of $\mathbf{2 0}$ assigned based on TROESY NMR technique, proved by X-ray diffraction.

\section{1-methyl-7-vinyl-8-oxa-bicyclo[3.2.1]octane (19)}

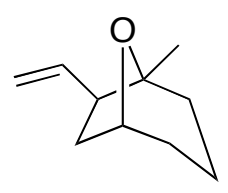

Flash chromatography (5\% EtOAc/hexanes) gave the pure product as a colourless oil (55\% yield), which gave satisfactory analytical data.

${ }^{1} \mathrm{H}$ NMR $\left(\mathrm{CDCl}_{3}, 400 \mathrm{MHz}\right): \delta 5.72(\mathrm{ddd}, J=17.1,9.8,9.8 \mathrm{~Hz}, 1 \mathrm{H}), 4.94-4.86(\mathrm{~m}, 2 \mathrm{H}), 4.36(\mathrm{~d}, J=7.6$ $\mathrm{Hz}, 1 \mathrm{H}), 2.58(\mathrm{ddd}, J=9.6,9.6,5.0 \mathrm{~Hz} 3 \mathrm{H}), 2.25(\mathrm{dd}, J=12.8,9.1 \mathrm{~Hz}, 1 \mathrm{H}), 1.82$ (ddd, $J=12.4,7.5,5.0$ $\mathrm{Hz}, 1 \mathrm{H}), 1.74-1.28(\mathrm{~m}, 6 \mathrm{H}), 1.12(\mathrm{~s}, 3 \mathrm{H})$;

${ }^{13} \mathrm{C} \mathrm{NMR}\left(\mathrm{CDCl}_{3}, 100 \mathrm{MHz}\right): \delta 141.9,113.3,81.7,75.0,49.9,38.4,38.0,30.1,23.7,17.0$;

37.9, 32.6, 25.1, 16.9, 14.9;

IR (neat): $\mathrm{cm}^{-1} 2937,1639,1446,1049$; 
GC-Mass: $\left[\mathrm{M}^{+}\right]=162$.

$[\alpha]^{25}-3.57\left(\mathrm{c}=0.3, \mathrm{CHCl}_{3}\right)$.

HPLC : Chiralcel OD, 7.5\% $\mathrm{iPrOH} / \mathrm{Hexane}, 0.50 \mathrm{~mL} / \mathrm{min}$., UV detection at $225 \mathrm{~nm}$, major peak at 16.8 min., minor peak at 26.2 min., $97 \%$ ee.

\section{6-methyl-1-phenyl-7-vinyl-8-oxa-bicyclo[3.2.1]octane (20)}

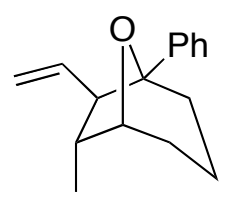

Flash chromatography (5\% EtOAc/hexanes) gave the pure product as a colourless oil (77\% yield), which gave satisfactory analytical data.

${ }^{1} \mathrm{H}$ NMR $\left(\mathrm{CDCl}_{3}, 400 \mathrm{MHz}\right): \delta 7.32-7.16(\mathrm{~m}, 5 \mathrm{H}), 5.10(\mathrm{ddd}, J=16.9,9.8,9.8 \mathrm{~Hz}, 1 \mathrm{H}), 4.80(\mathrm{dd}, J=$ 16.9, $2.1 \mathrm{~Hz}, 2 \mathrm{H}), 4.66(\mathrm{dd}, J=9.8,2.1 \mathrm{~Hz}, 1 \mathrm{H}), 4.30(\mathrm{bs}, 1 \mathrm{H}), 2.50(\mathrm{dd}, J=9.7,7.2 \mathrm{~Hz}, 3 \mathrm{H}), 2.15$ (dddd, $J=7.1,7.1,7.1,7.1 \mathrm{~Hz}, 1 \mathrm{H}), 2.00-1.60(\mathrm{~m}, 6 \mathrm{H}), 1.13(\mathrm{~d}, J=7.2 \mathrm{~Hz}, 3 \mathrm{H})$;

${ }^{13} \mathrm{C} \mathrm{NMR}\left(\mathrm{CDCl}_{3}, 100 \mathrm{MHz}\right): \delta 141.9,113.3,81.7,75.0,49.9,38.4,38.0,30.1,23.7,17.0$;

37.9, 32.6, 25.1, 16.9, 14.9;

IR (neat): $\mathrm{cm}^{-1} 2917,1637,1446,1052$;

HRMS (EI) calcd for $\mathrm{C}_{16} \mathrm{H}_{20} \mathrm{O}: 228.1514$; found: 228.1515.

$[\alpha]_{D}^{25}+30.08\left(\mathrm{c}=0.35, \mathrm{CHCl}_{3}\right)$.

HPLC : Chiralcel OD, Hexane, $1.00 \mathrm{~mL} / \mathrm{min}$., UV detection at $254 \mathrm{~nm}$, major peak at $7.0 \mathrm{~min}$., minor peak at 3.6 min., $98 \%$ ee. 


\section{Copies of ${ }^{1} \mathrm{H}$ and ${ }^{13} \mathrm{C}$ NMR spectra}
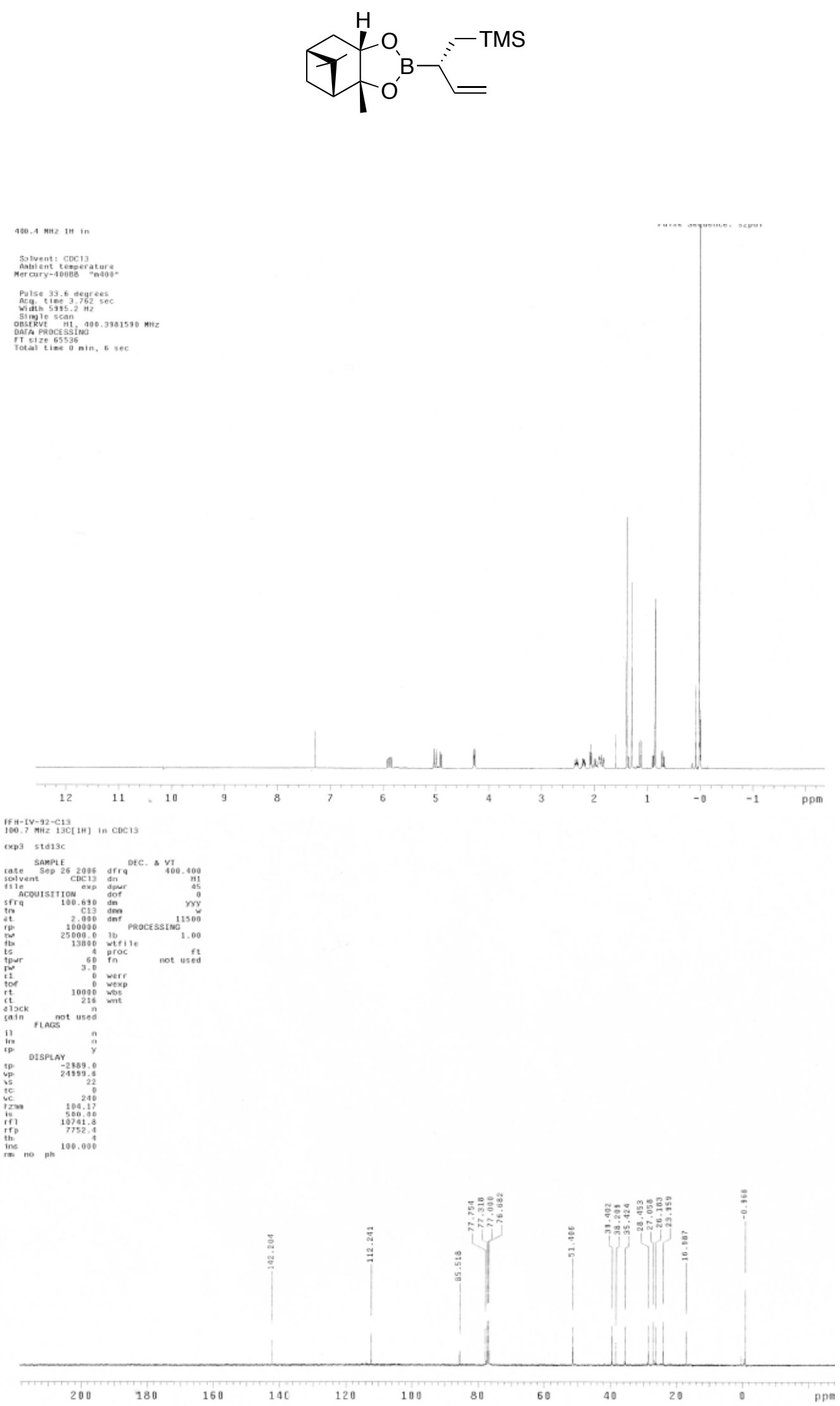
$\mathrm{THO}^{\mathrm{O}}=\mathrm{C}^{\mathrm{TMS}}$

PFH-TV-201
400 HHZ 10 in $\mathrm{CDC}_{13}$ (ref, to CDC13 7.24 pps)

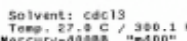

Rogax. atolav 0.100 sec

Puise. $45.0 \% 169806$

16 repetitions 3901559 niz

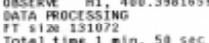

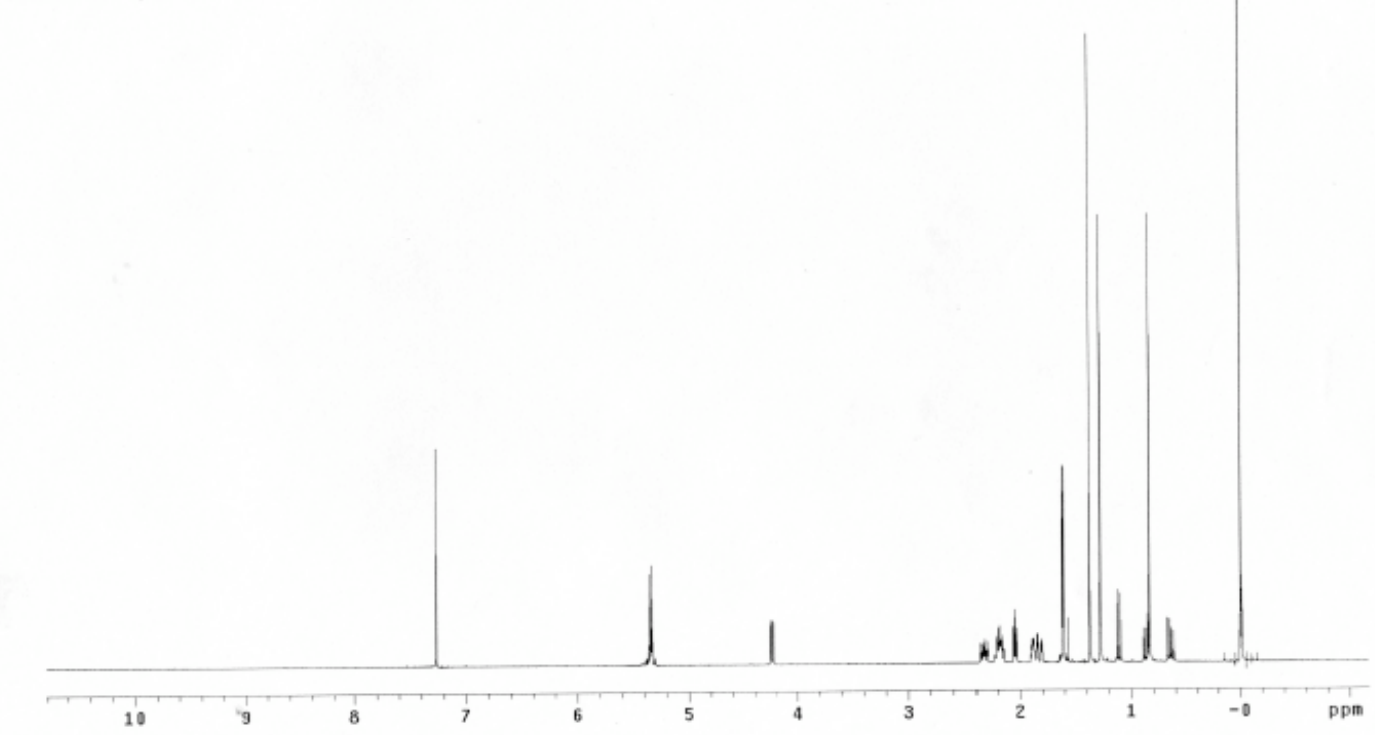

$\mathrm{PFH}-1 / 201-\mathrm{C}_{13}$
100.7 fiz $13 \mathrm{C}[1 \mathrm{H}]$ in $\mathrm{CDC}_{13}$

exp4 sta13e
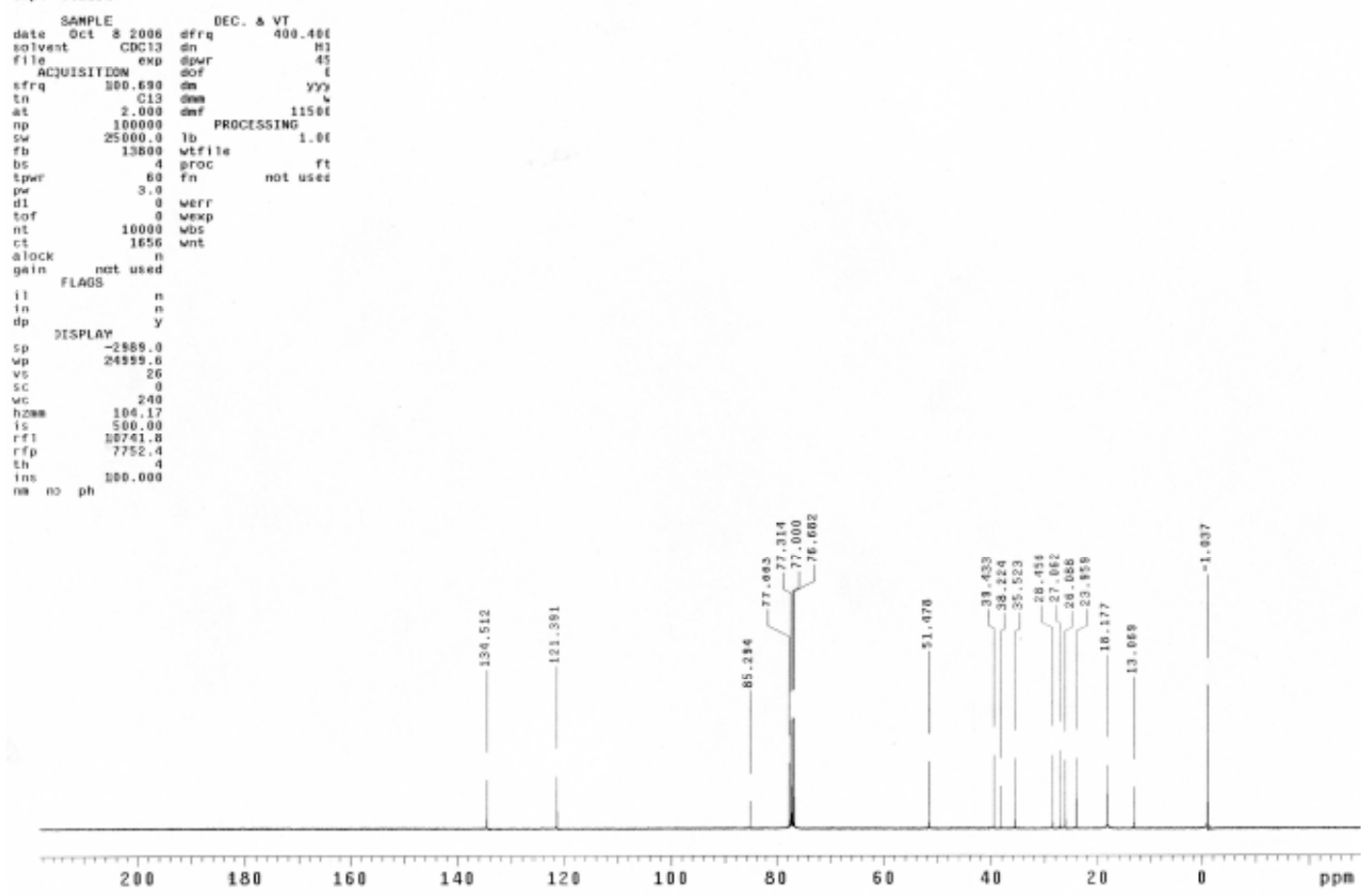


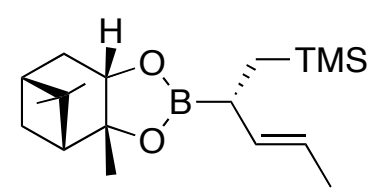

PrH-
$400-100$
MHZ

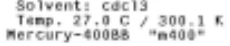

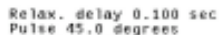

Acq. tine 5.002 sec

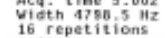

OBSERV H1, 400.398165, MHZ

OATA PROCESSINO
TT 5120131072

TT $51 z 0131072$
Totai time 1 ain, 50 sec

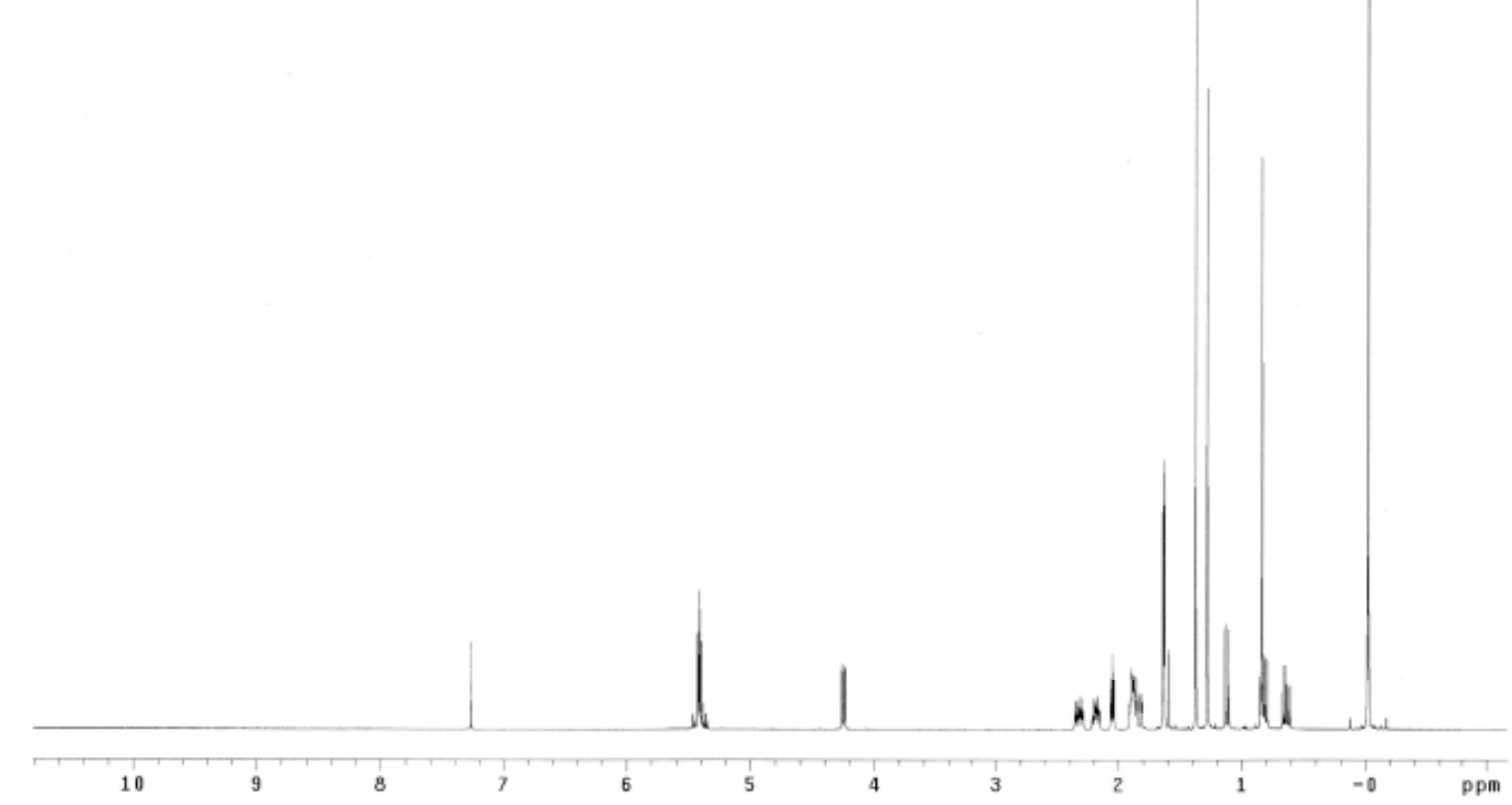

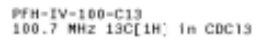

$\operatorname{exp2} s t e 13 c$
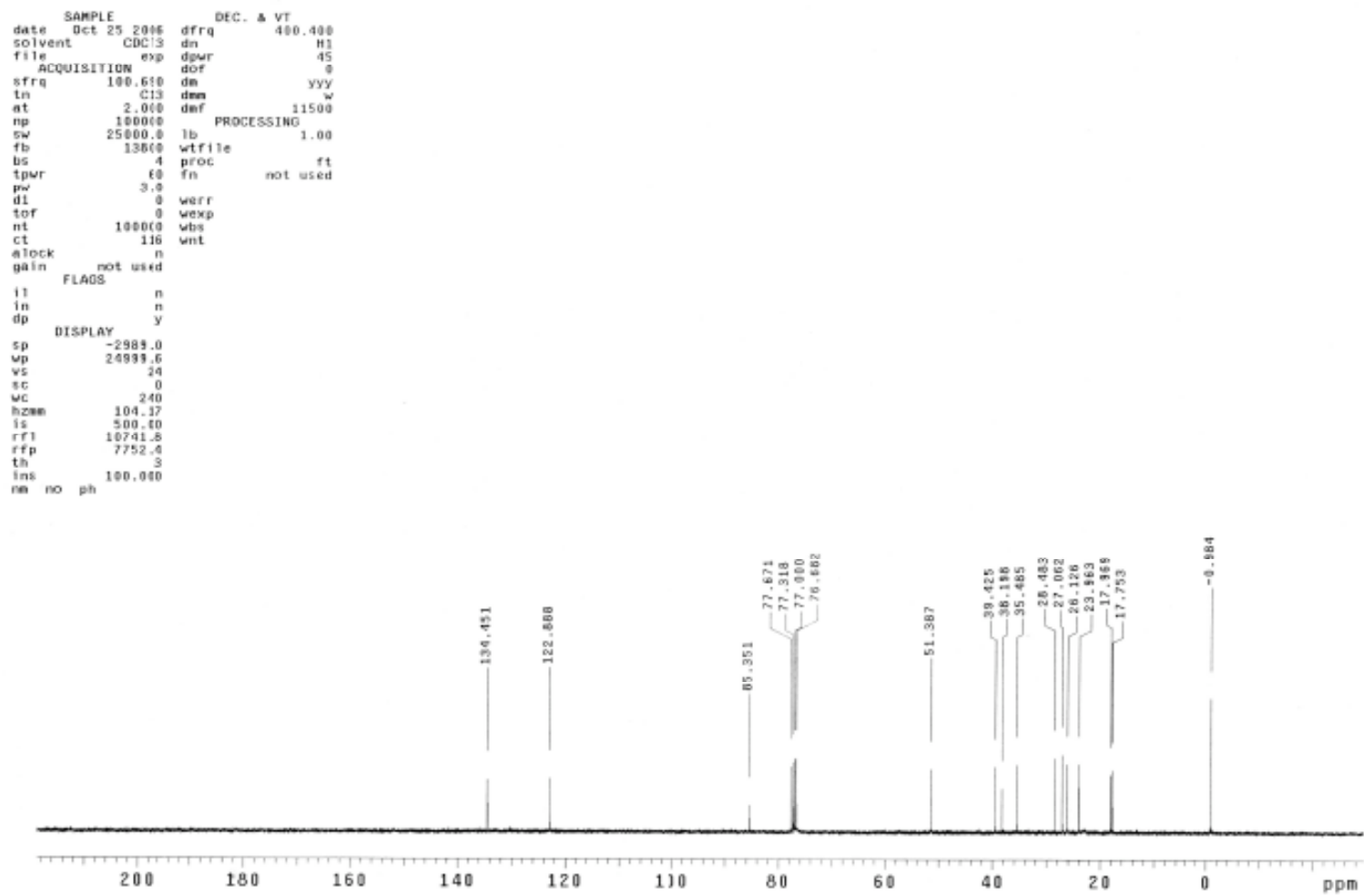
$(R, E)$-7-(trimethylsilyl)-1-phenylhept-5-en-3-ol (3a)

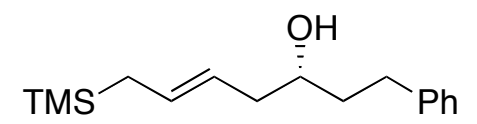

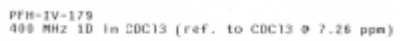
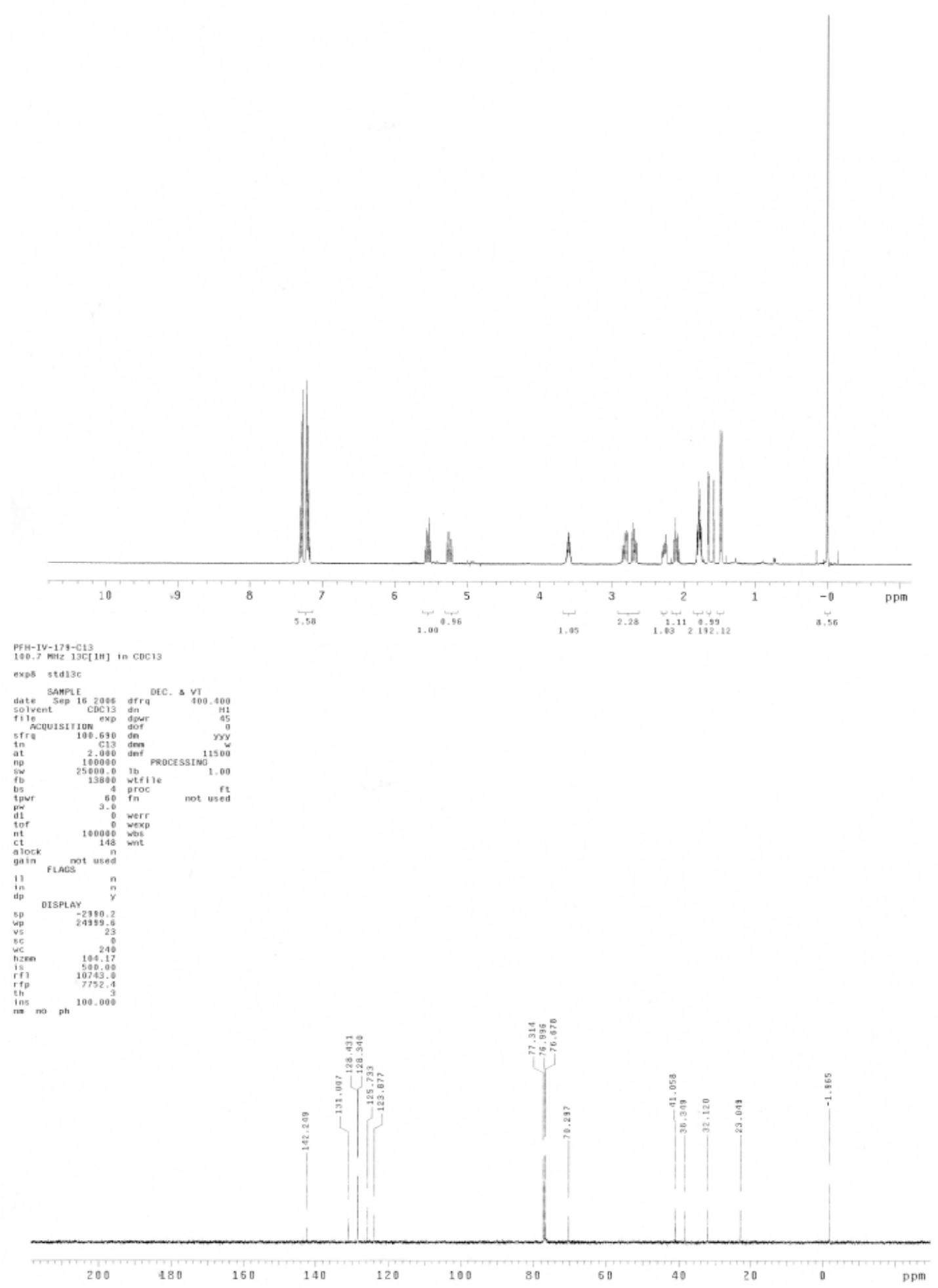
(R,E)-1-(trimethylsilyl)dodec-2-en-5-ol (3b)

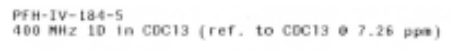
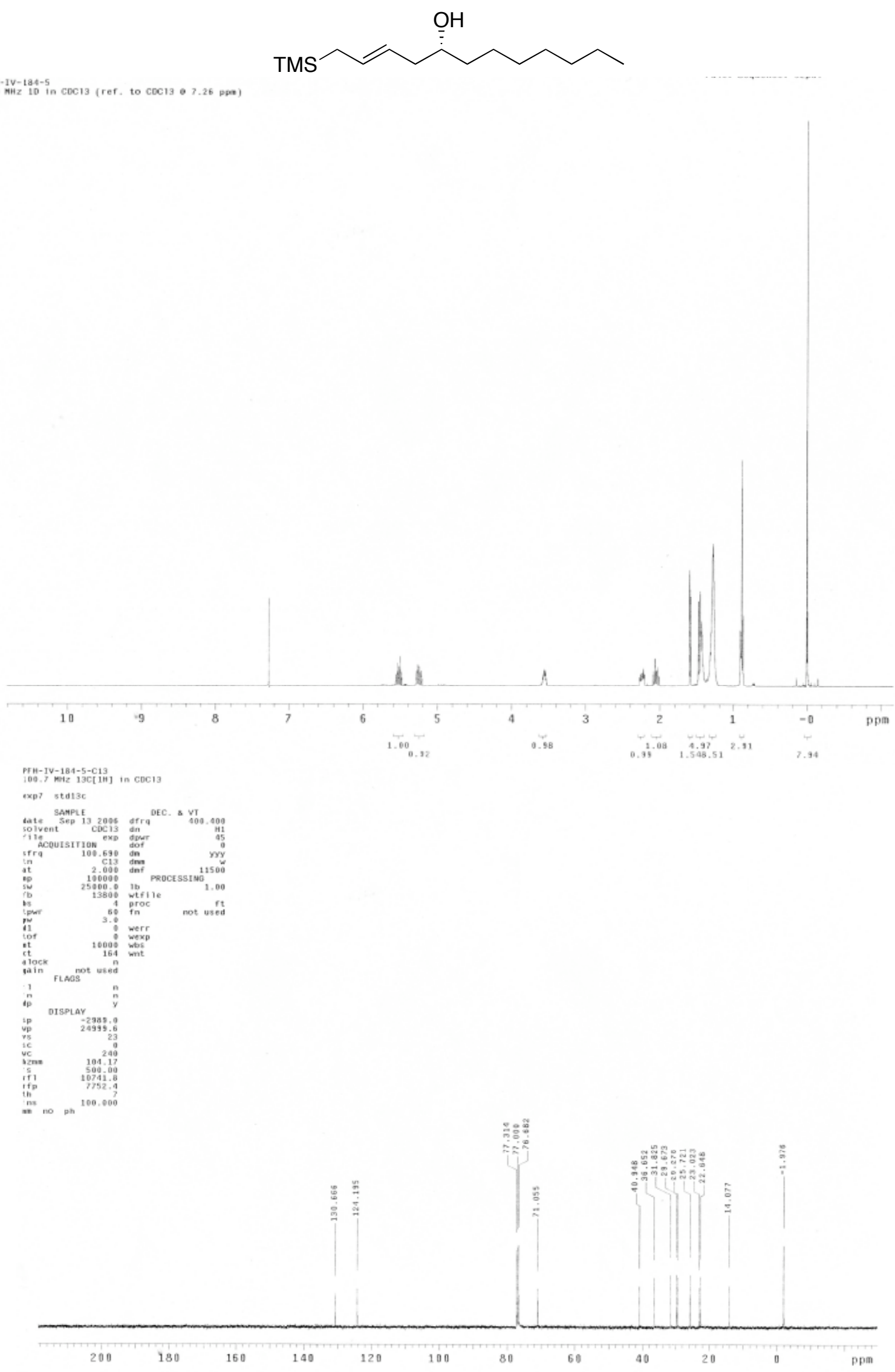
(S,E)-2-methyl-7-(trimethylsilyl)hept-5-en-3-ol (3c)

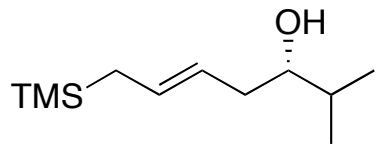

PrH-TV-184-6
$S 60$

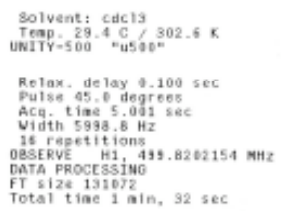

FT size 1319072 .

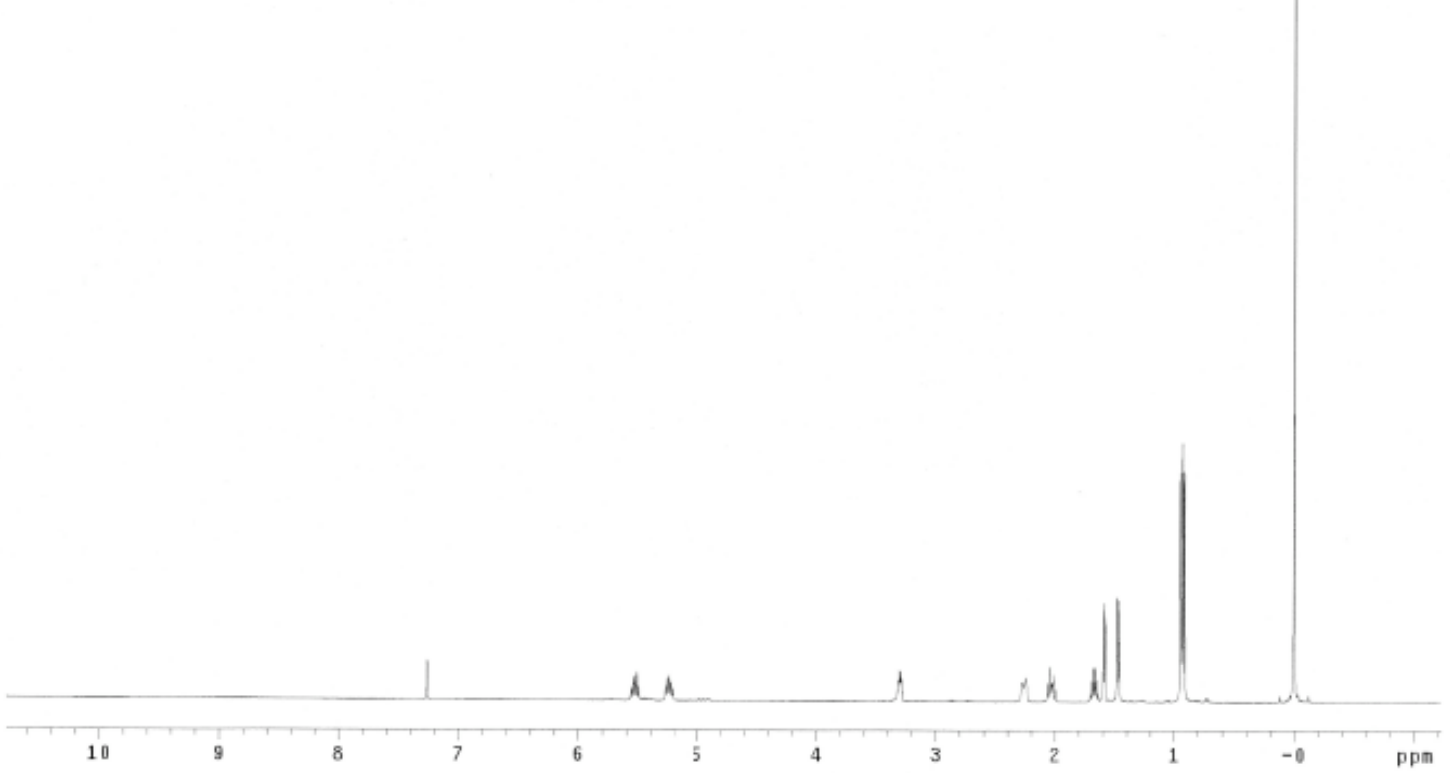

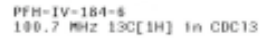

exp7 statist

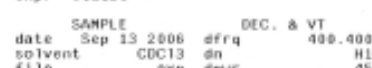

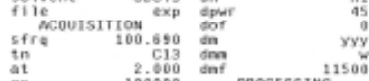

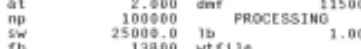

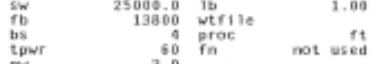

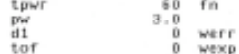

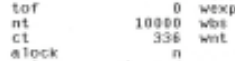

gain in mot used

in

dip DISPLAY y

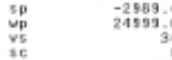

uc
nzem
is
is

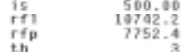

${ }_{\text {ins }}^{\text {ins on }}{ }^{100.000^{3}}$

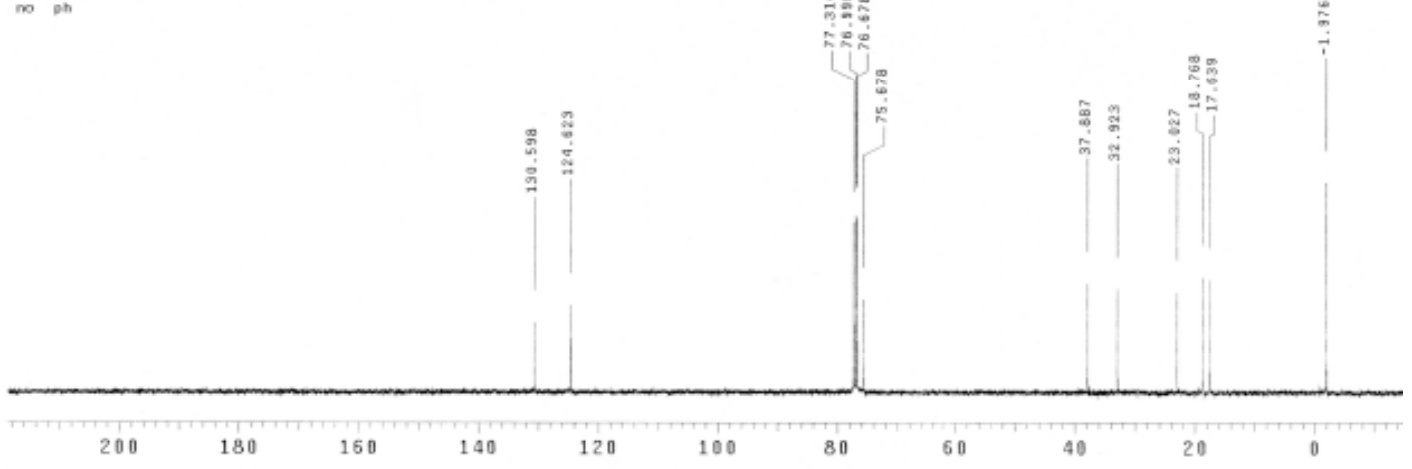


(S,E)-1-(benzyloxy)-6-(trimethylsilyl)hex-4-en-2-ol (3d)

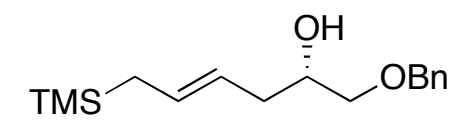

PFH-1r-205
400
Hex

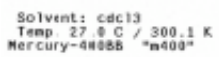

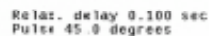

Acq, time 5.002 .

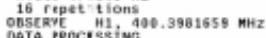

TT 51200131072
Total time 1 in
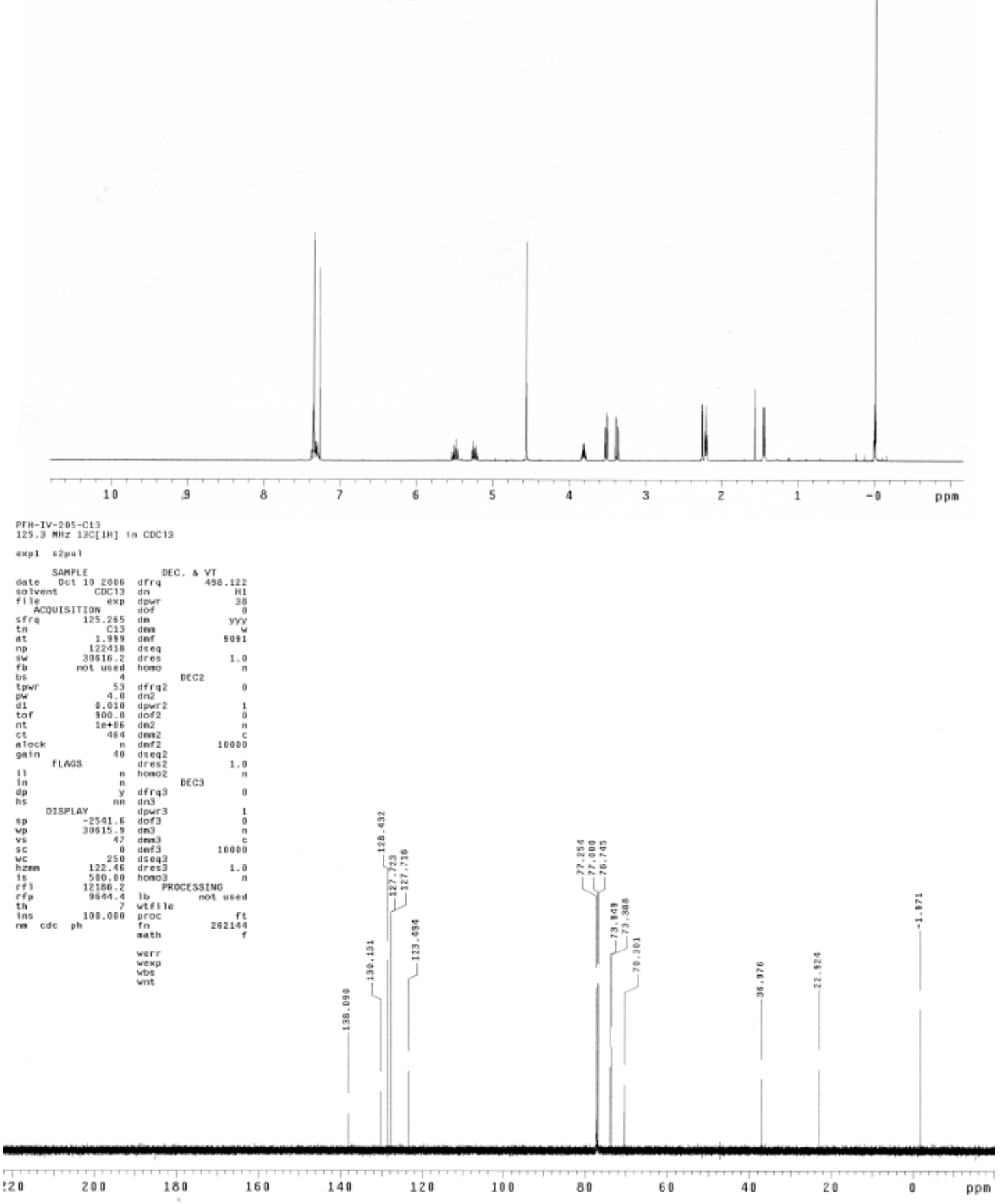
(S,E)-5-(trimethylsilyl)-1-phenylpent-3-en-1-ol (3e)

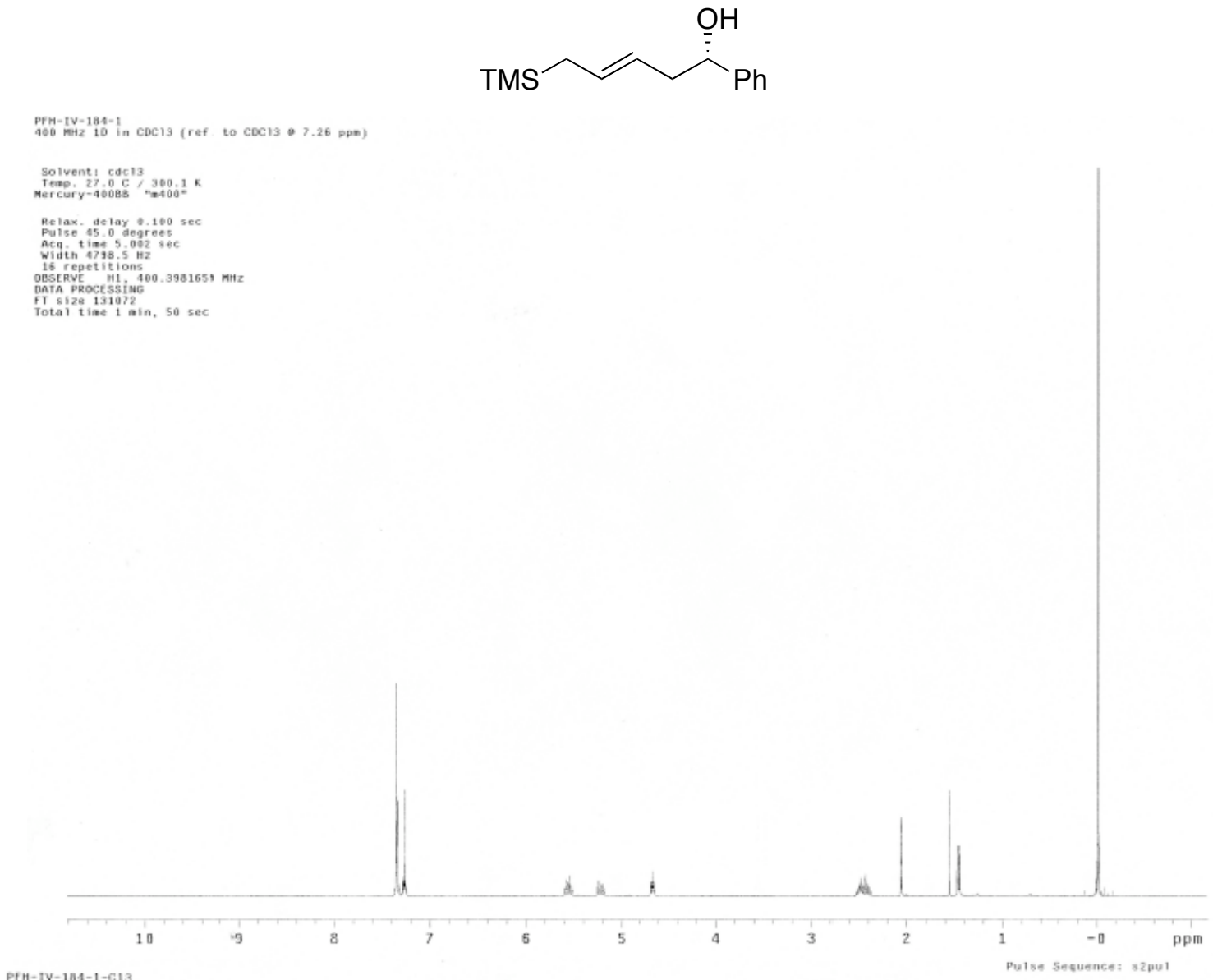

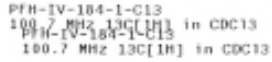

expl sta13e
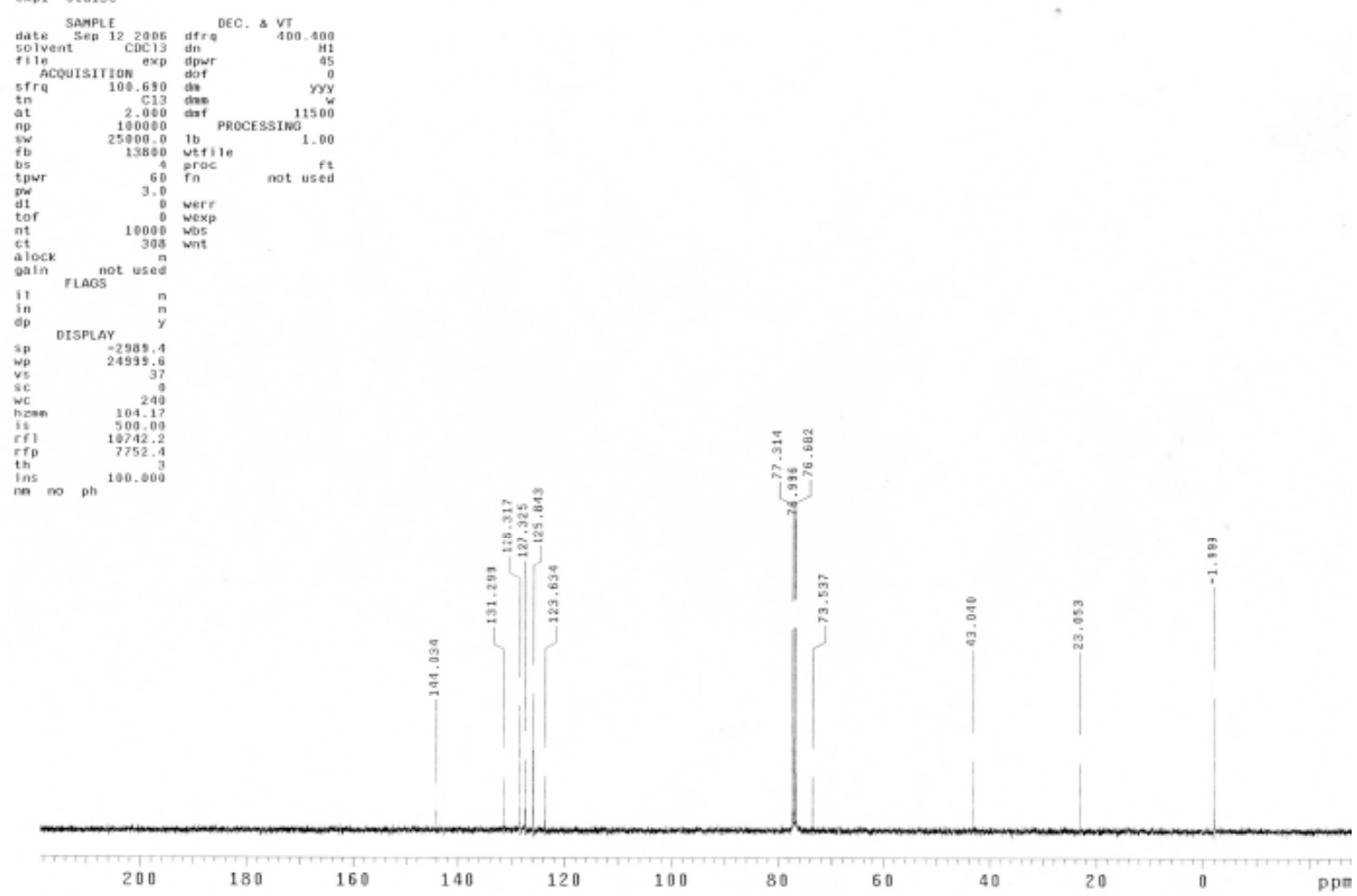
$(S, 1 E, 5 E)$-7-(trimethylsilyl)-1-phenylhepta-1,5-dien-3-ol (3f)

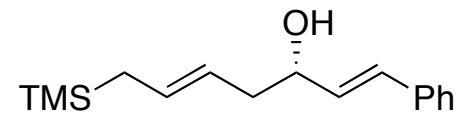

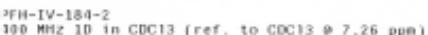

Solvent: cacis
Teap. $27.0 \mathrm{C}$ C $300-1 \mathrm{~K}$
Mercury $=40000$

Relax, delay 0,100 vec

Ach tine 5,002 sec

NBSERVE H1, 400.3981659 MH:

TATA PROCE:

Total time inin, so sec

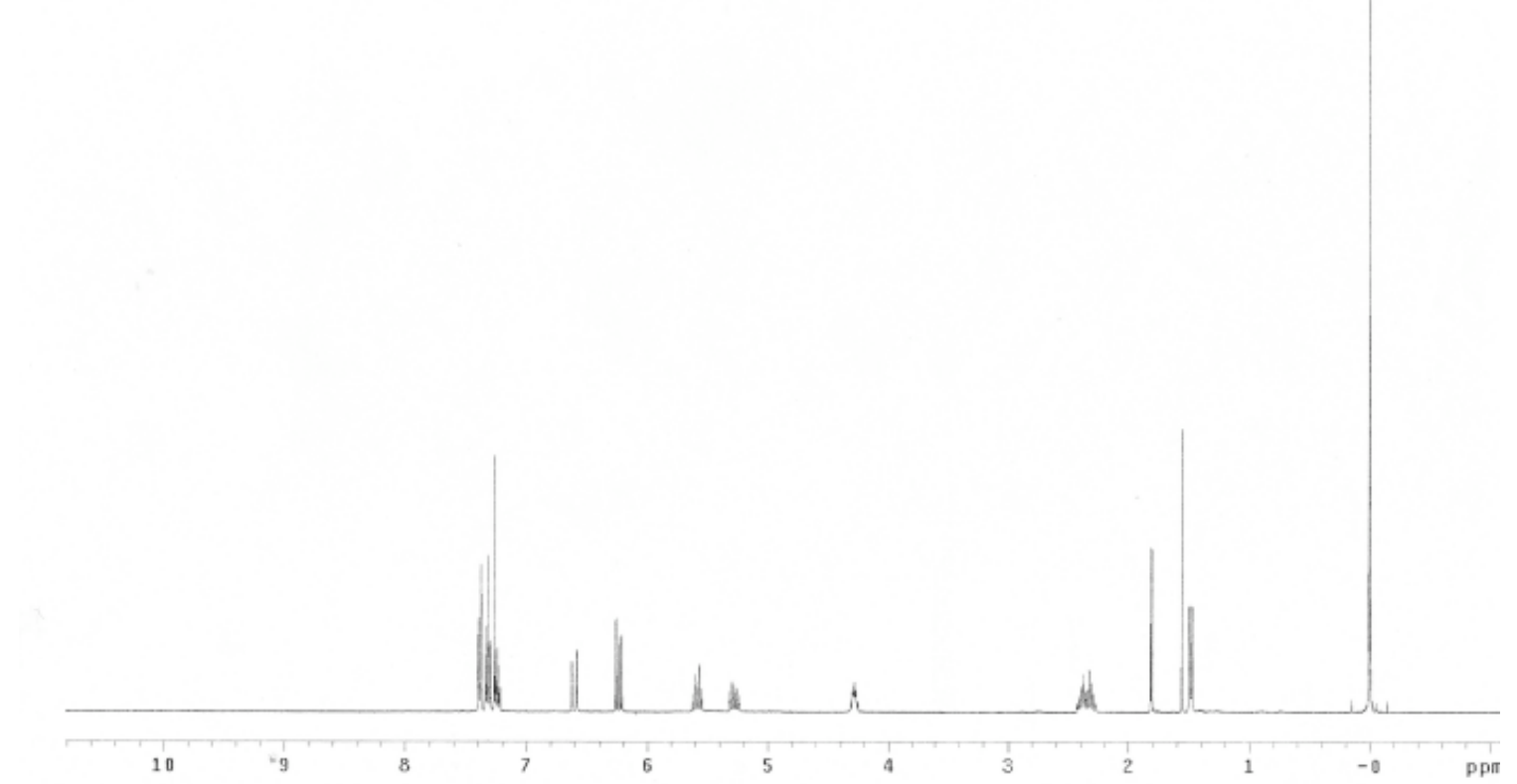

PFH-IV-184-2-C13
$100.7 \mathrm{MHz} 13 \mathrm{CH}[1 \mathrm{H}]$ in $\mathrm{COC} 13$

$\exp 3 \operatorname{std13c}$
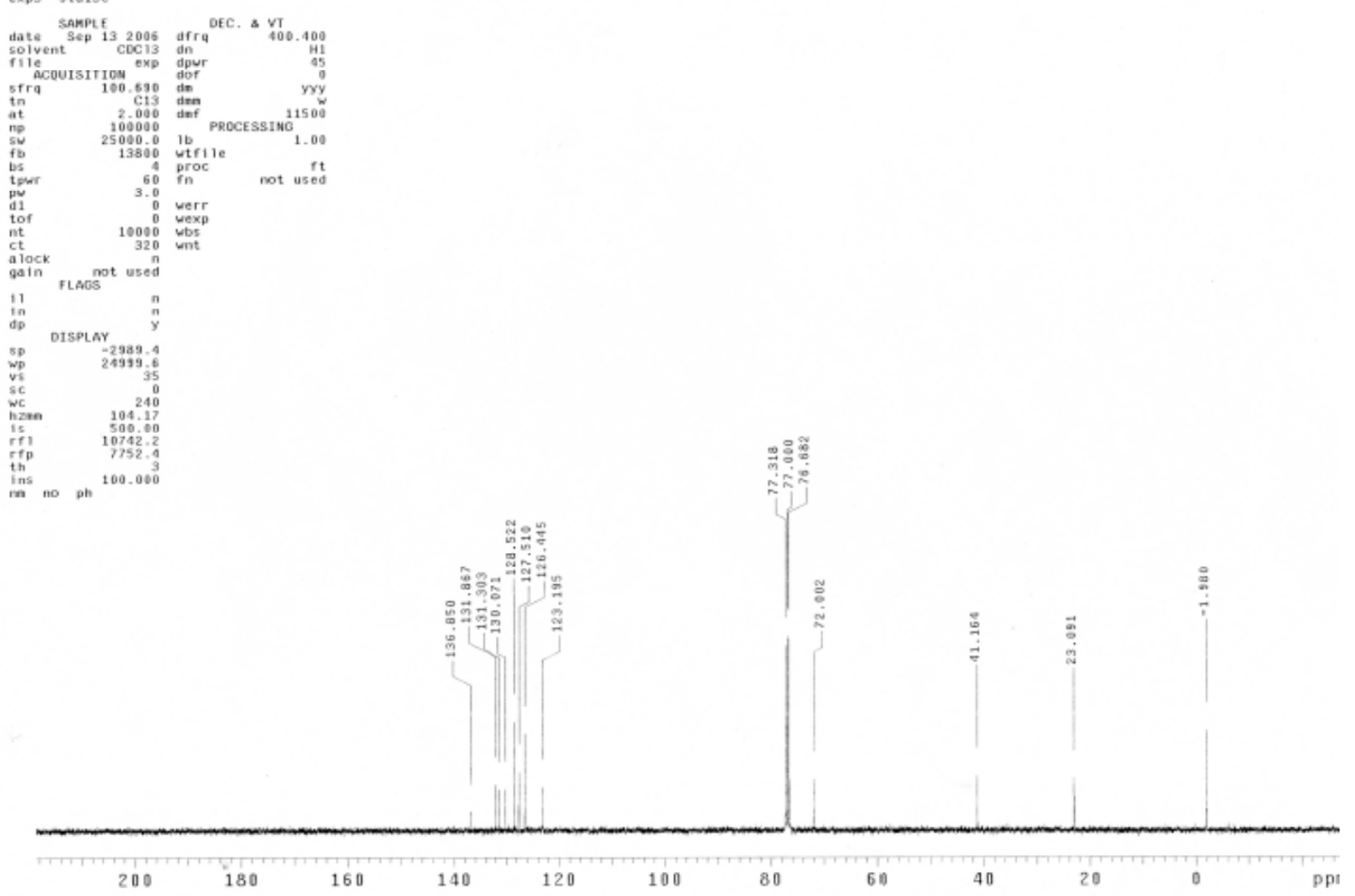


\section{$(S, 2 E, 6 E)-8$-(trimethylsilyl)octa-2,6-dien-4-ol (3g)}
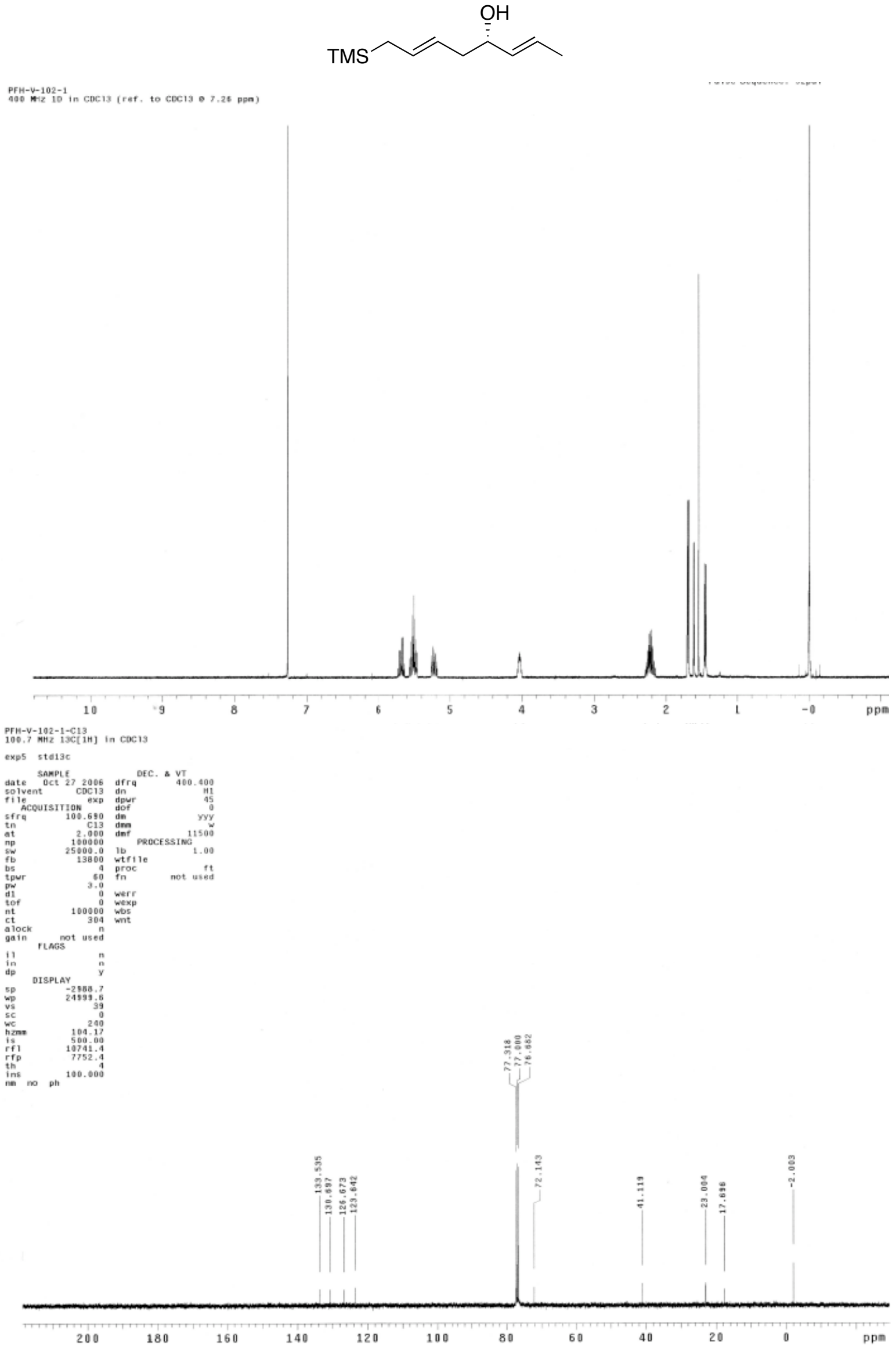


\section{(S,E)-1-(trimethylsilyl)dodec-2-en-6-yn-5-ol (3h)}
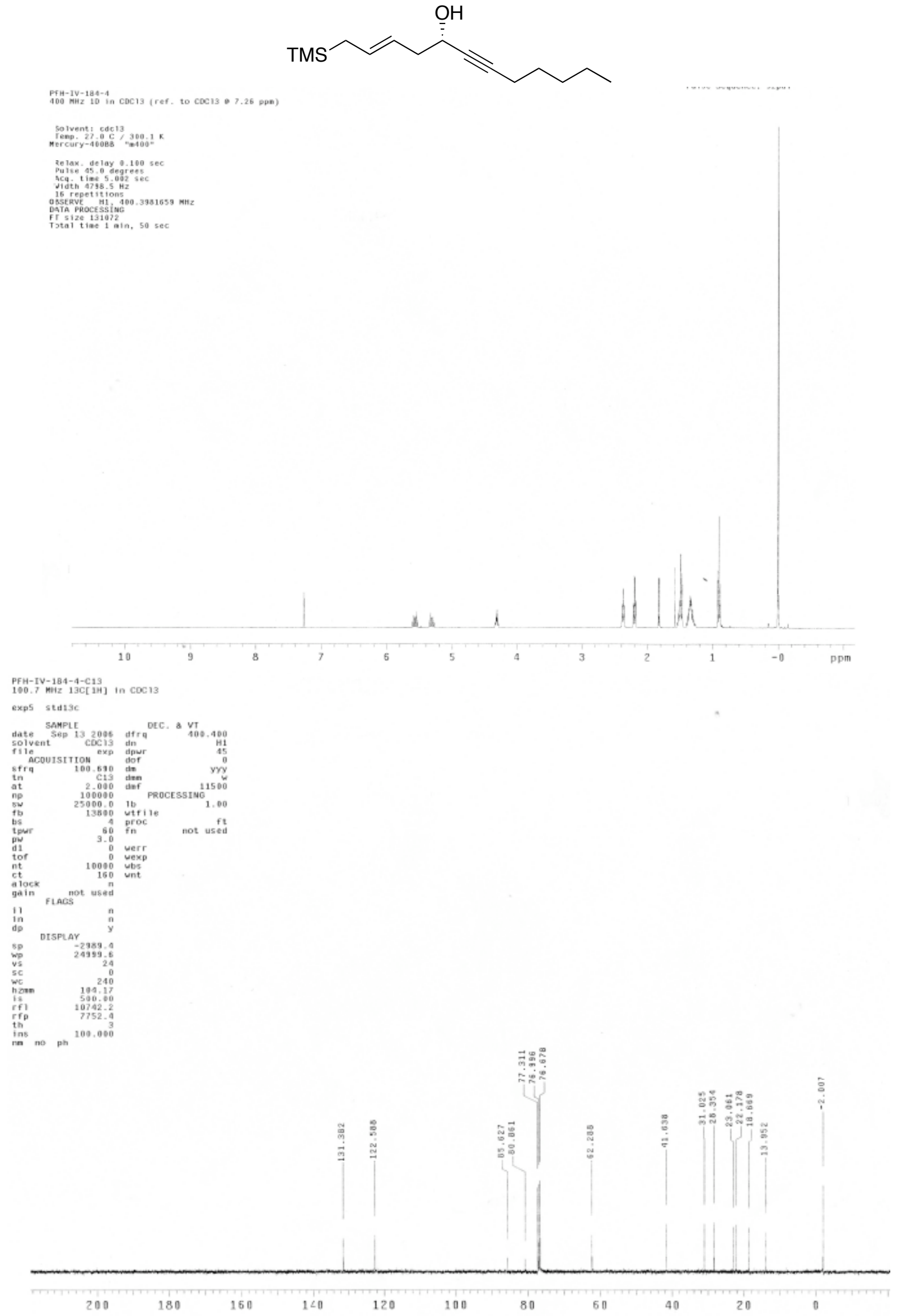
$(E, 3 R, 4 R)$-4-methyl-7-(trimethylsilyl)-1-phenylhept-5-en-3-ol (13)

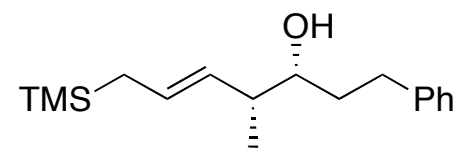

fFH-IV-202-1
$400 \mathrm{WHz}$
$\mathrm{hH}$

Putse sequencer 52pul

Solvent: cdc1s
Teap. $27.0 \mathrm{C}, 300.1 \mathrm{~K}$
nercury-4008

Relax. delay $0.100 \mathrm{sec}$

Acy ${ }^{\mathrm{t} i n e} 5.002$ sec

16 repetition

(1)

Fin 5126131072
Total tiec 1 ain, $50 \mathrm{sec}$
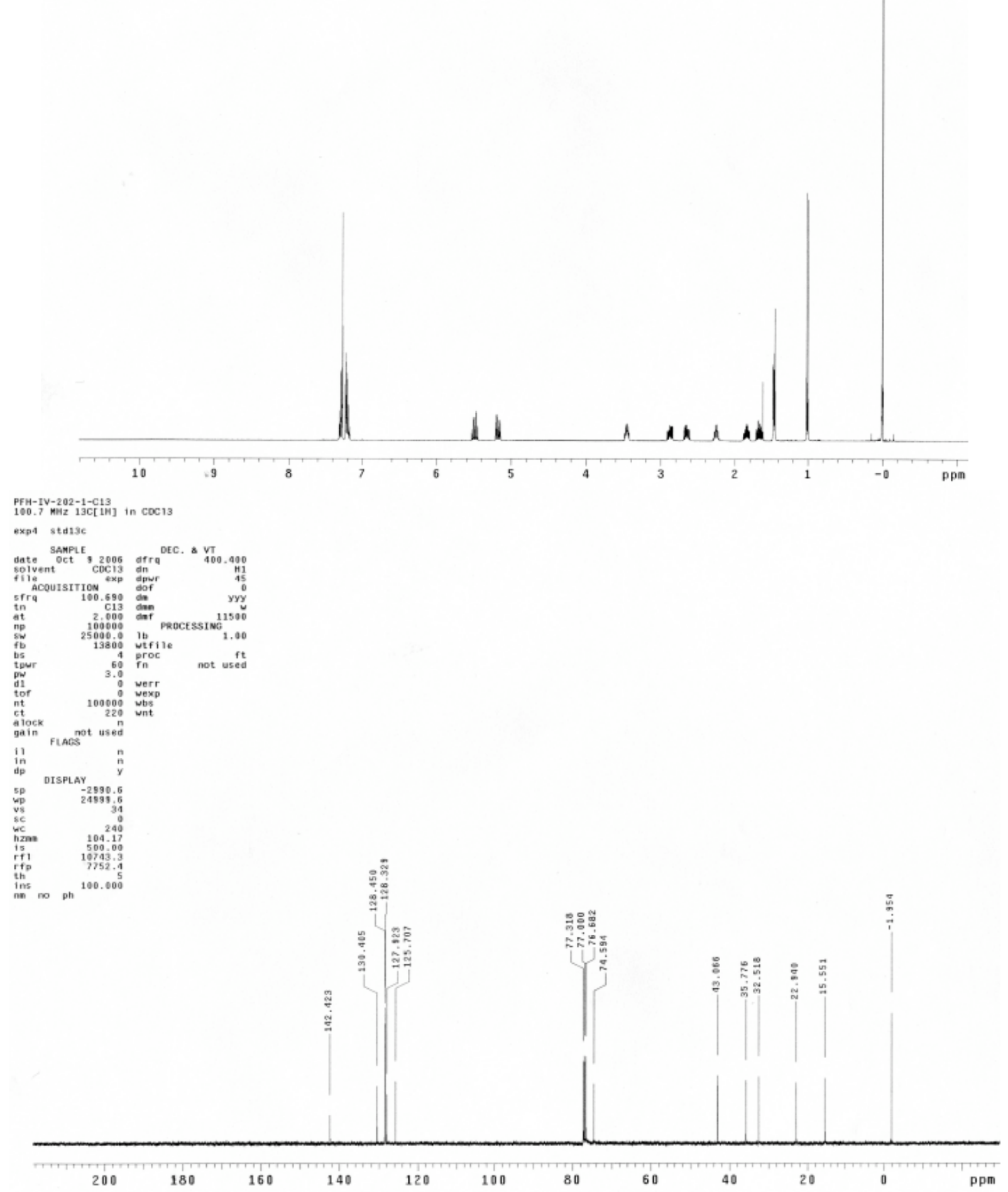
$(E, 2 S, 3 R)$-1-(benzyloxy)-3-methyl-6-(trimethylsilyl)hex-4-en-2-ol (14)
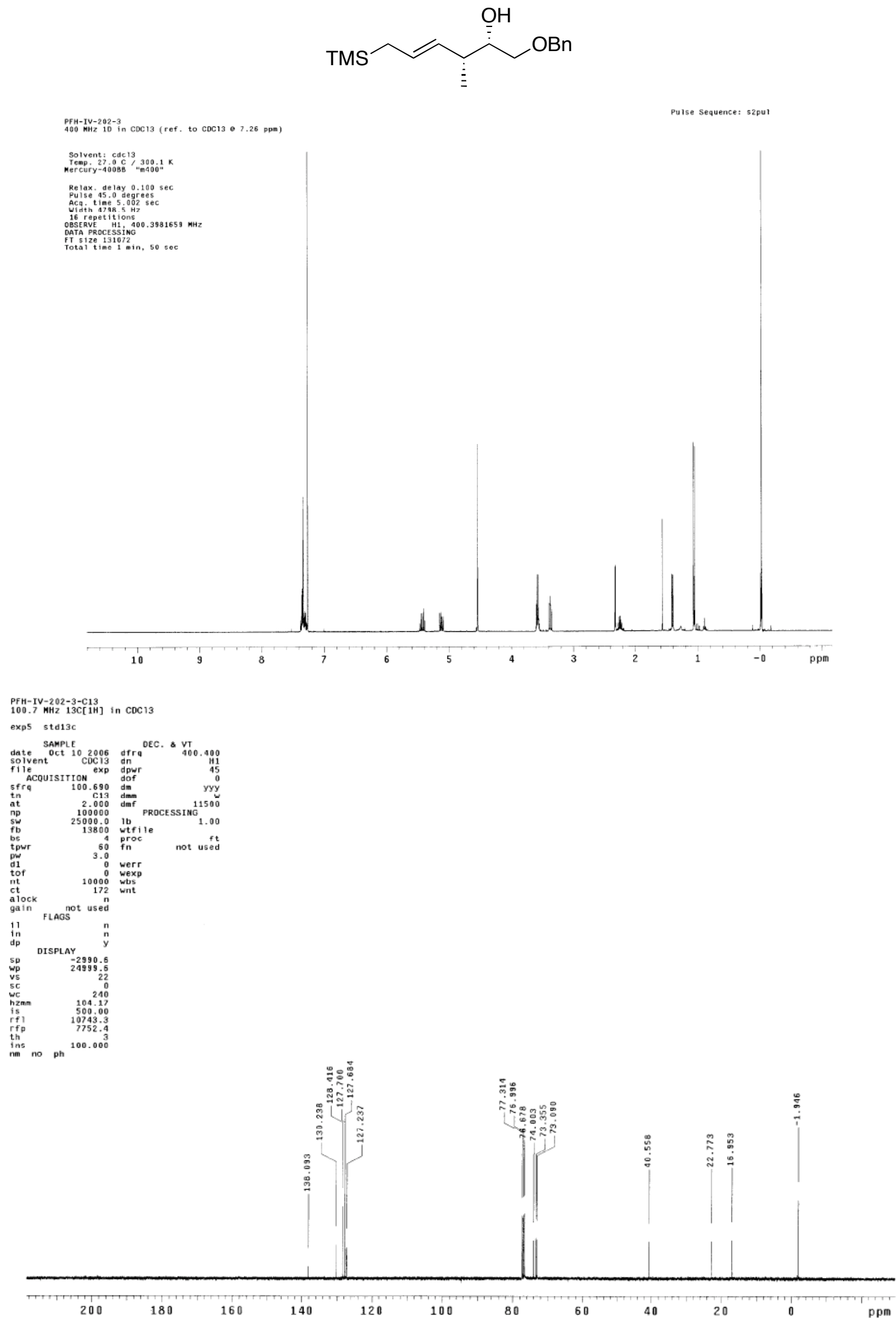
$(E, 1 S, 2 R)$-2-methyl-5-(trimethylsilyl)-1-phenylpent-3-en-1-ol) (15)
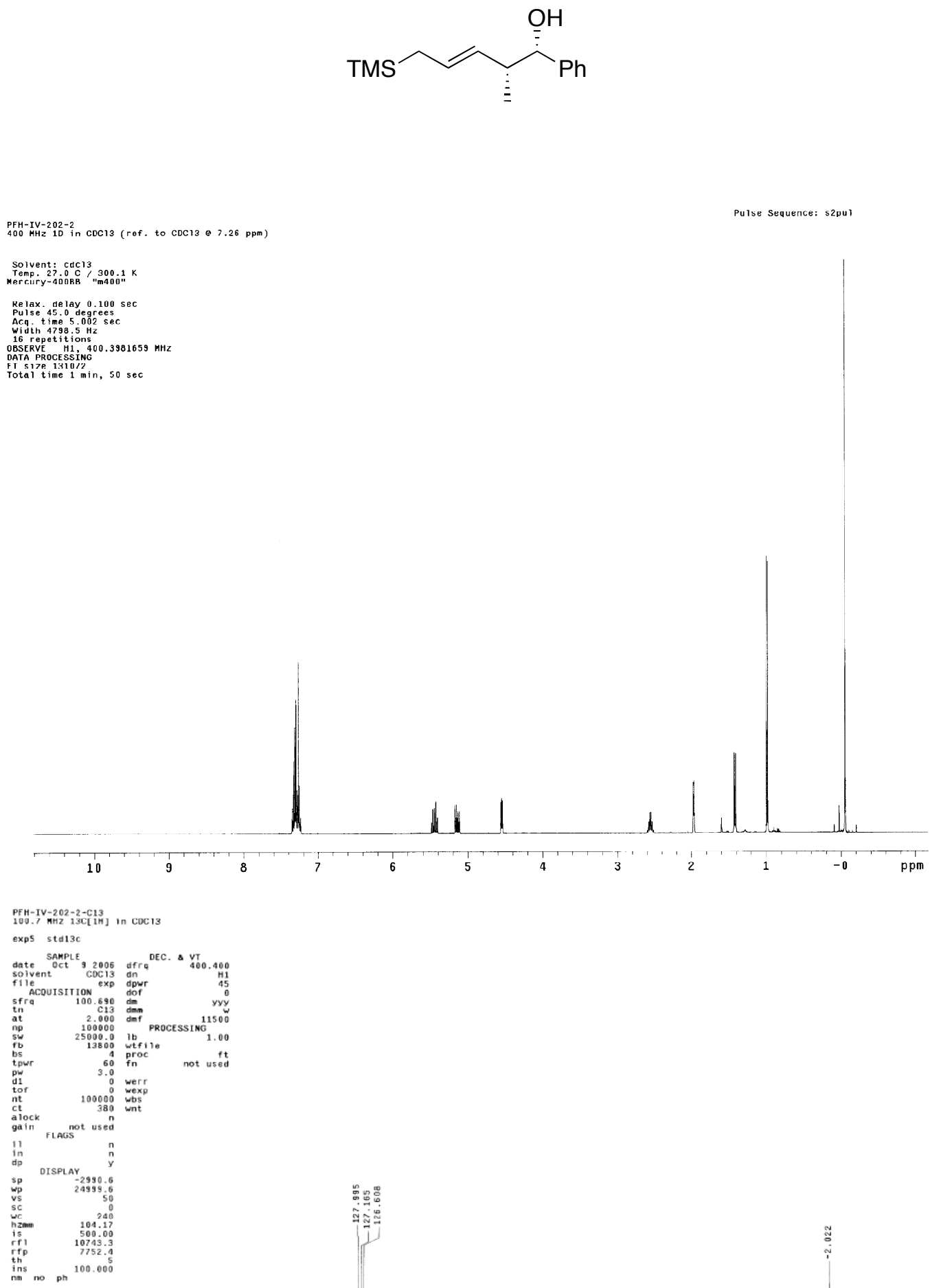
$(E, 3 R, 4 S)$-4-methyl-7-(trimethylsilyl)-1-phenylhept-5-en-3-ol (16)
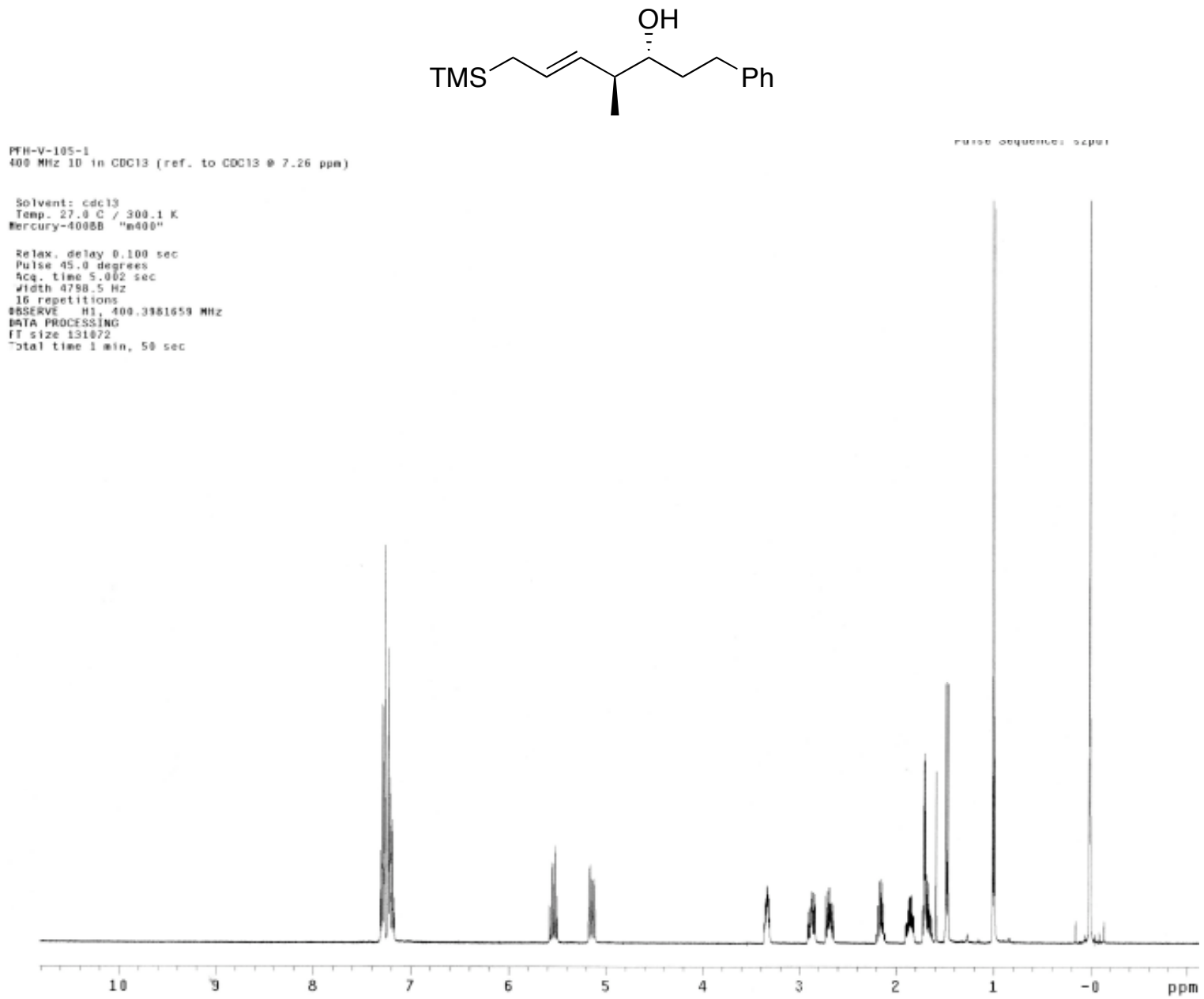

PfH-V-105-1-C13
$100-7$ MHz $13 \mathrm{C}[1 \mathrm{H}]$ in $\mathrm{COC}^{3}$

expl sta13c
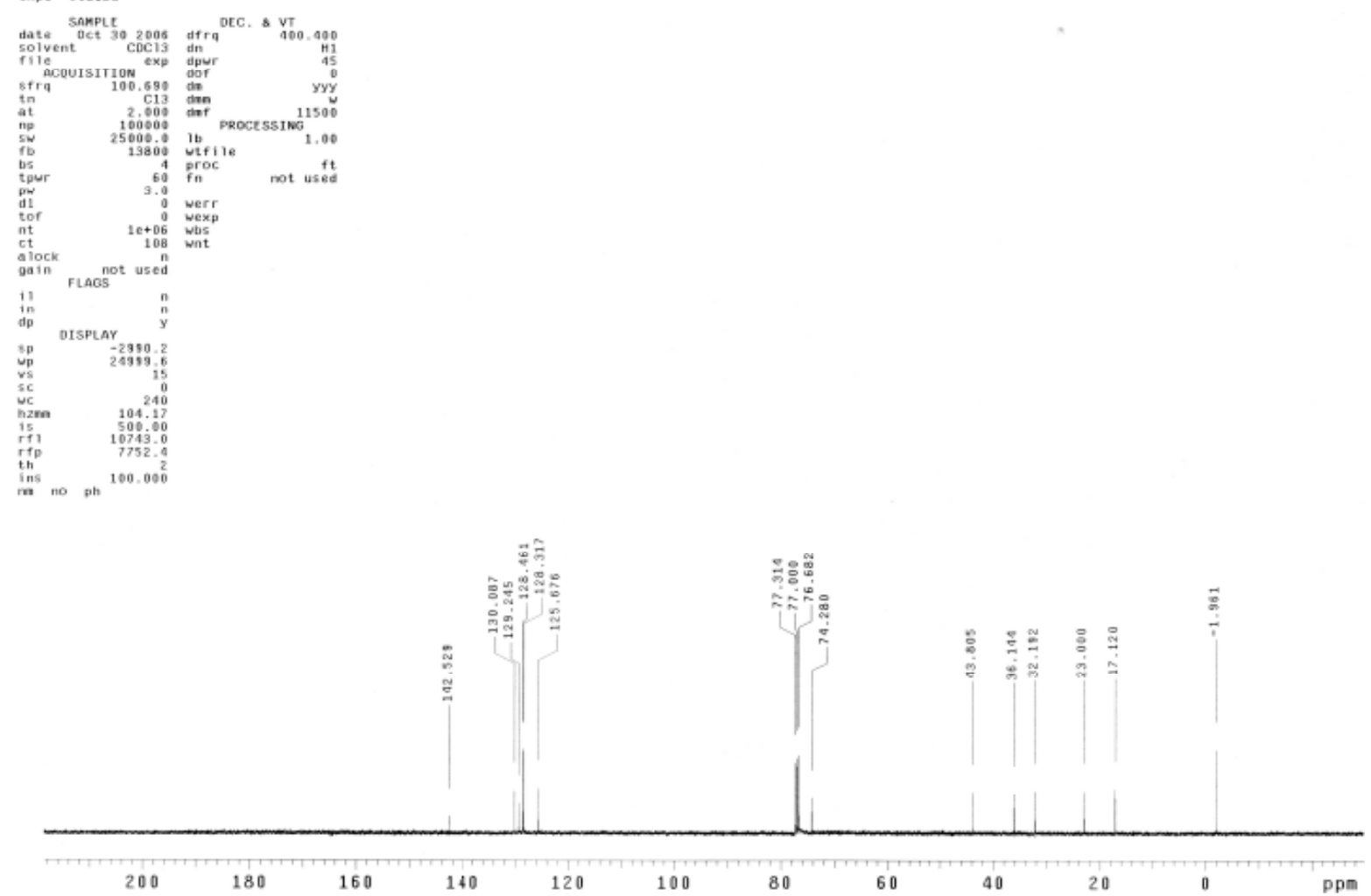
(E,2S,3S)-1-(benzyloxy)-3-methyl-6-(trimethylsilyl)hex-4-en-2-ol (17)

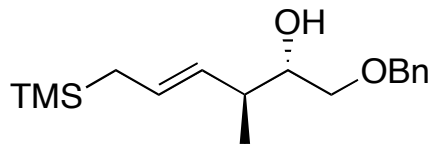

PrH
$400 \mathrm{~V}-105-3$
$\mathrm{NHz}$

Pulse Sequence: s2pul

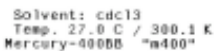

Relax, de lay 0.100 sec

Acc. tine 5.002 sec

is repstitions

QESERVt ${ }_{1}$

TT 5 Ize 131072
Total tiae 1 nin, 50 sec

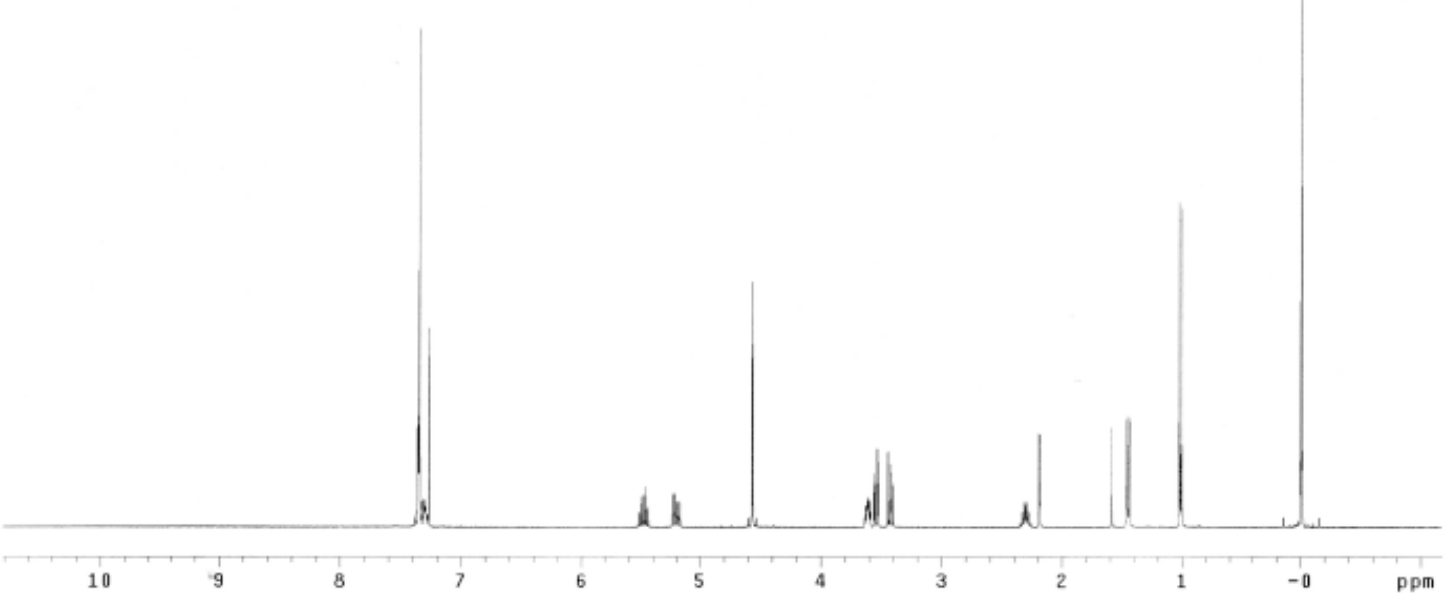

$\mathrm{PFH}-\mathrm{V}-105-9-\mathrm{C} 13$
100.7 mHz $13 \mathrm{C}[1 \mathrm{1}]$ in $\mathrm{COC} 13$

exp1 stdise
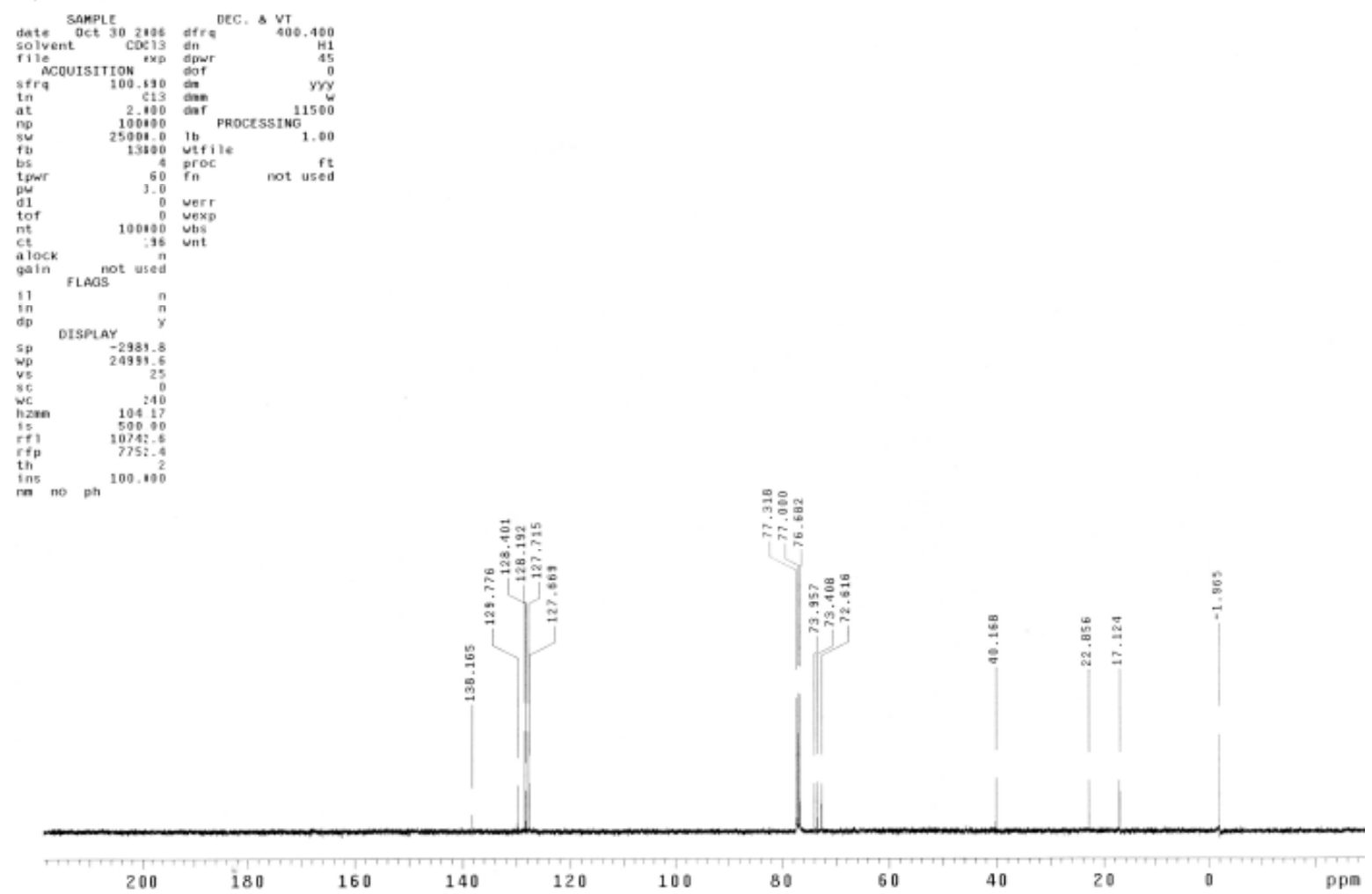
$(E, 1 S, 2 S)$-2-methyl-5-(trimethylsilyl)-1-phenylpent-3-en-1-ol) (18)
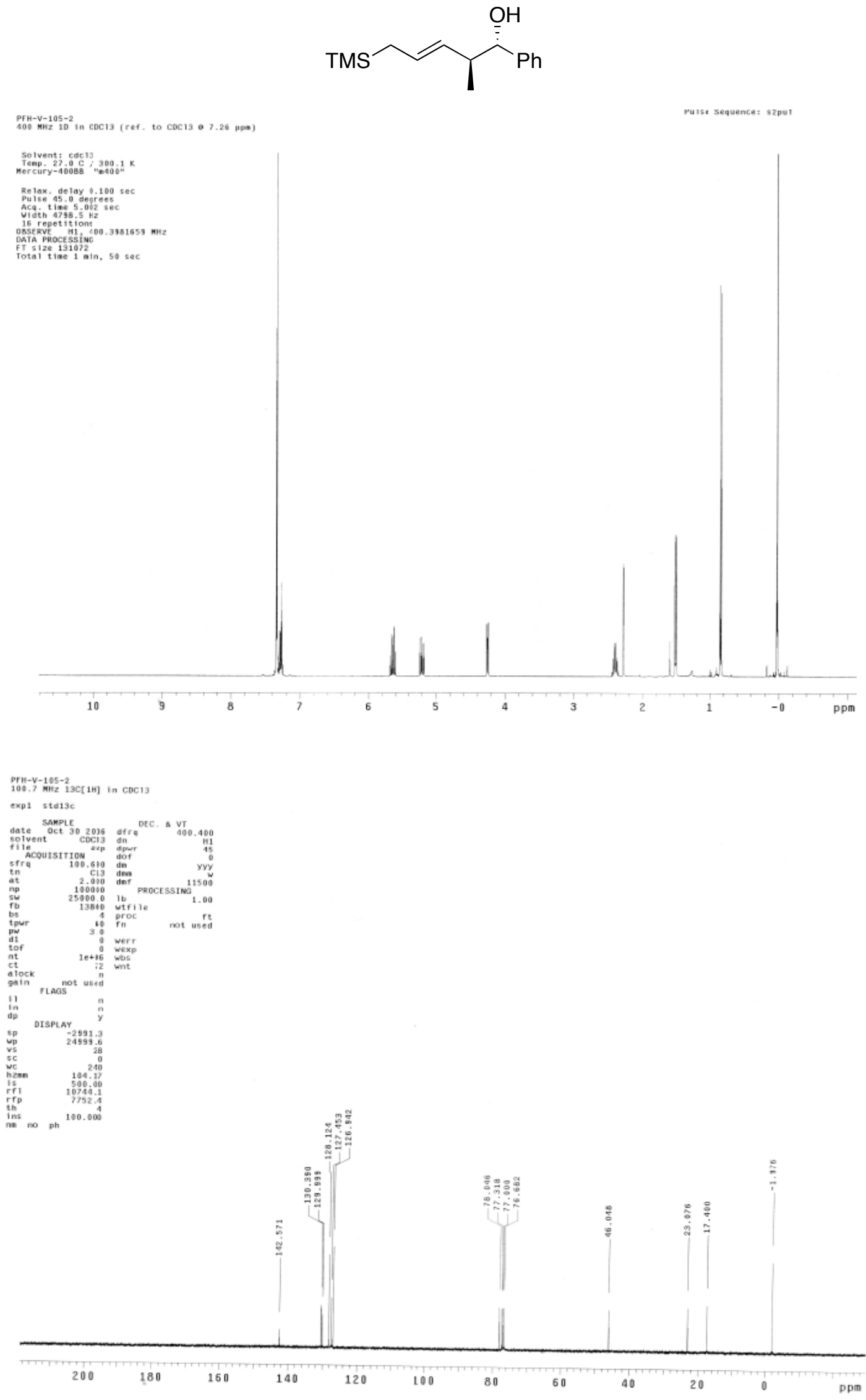
tetrahydro-5-phenethyl-2-phenyl-3-vinylfuran (5)

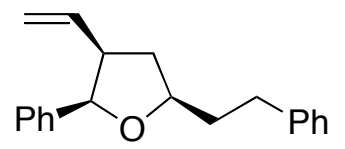

Solvent 1 cdec 13
Teap. $27.5 \mathrm{c} / 300.6 \times$

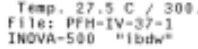

Relax. de lay $9.000 \mathrm{sec}$

Acq. tine 2.001 sec

Width 3597 . $6 \mathrm{~Hz}$

OBSERVE H1, 293.3596505 MHZ

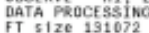

F size 131072
Total tine 1 in, 30 sec

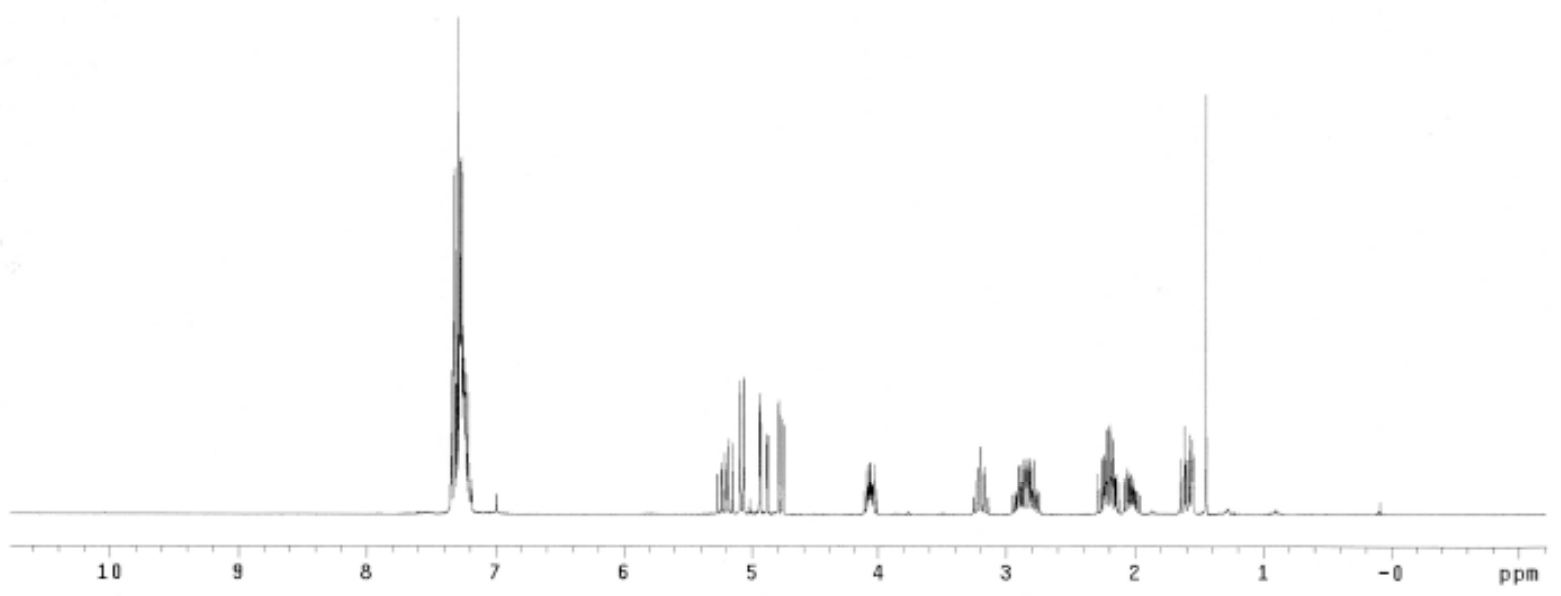

PFH-IV- $153-1-\mathrm{Cl}_{13}$
$100.7 \mathrm{MHz}$
$13 \mathrm{C}[1 \mathrm{H}]$ in $\mathrm{COC} 13$

exp2 stat3c

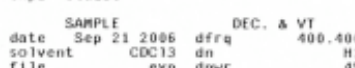

Solvent
ril

$\begin{array}{ll}51 \mathrm{ra} & 100.690 \\ \text { tn } & \end{array}$

fo

tpor

$$
\begin{aligned}
& \text { tor } \\
& \text { tor } \\
& \text { nt } \\
& \text { ct } \\
& \text { ald } \\
& \text { ato }
\end{aligned}
$$$$
\begin{gathered}
\text { ct ack } \\
\text { aloin } \\
\text { cain }
\end{gathered}
$$

gain FLaCS

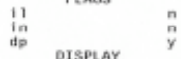

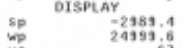

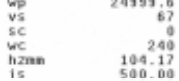

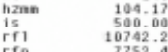

in $_{\text {ins no ph }} 100.000$

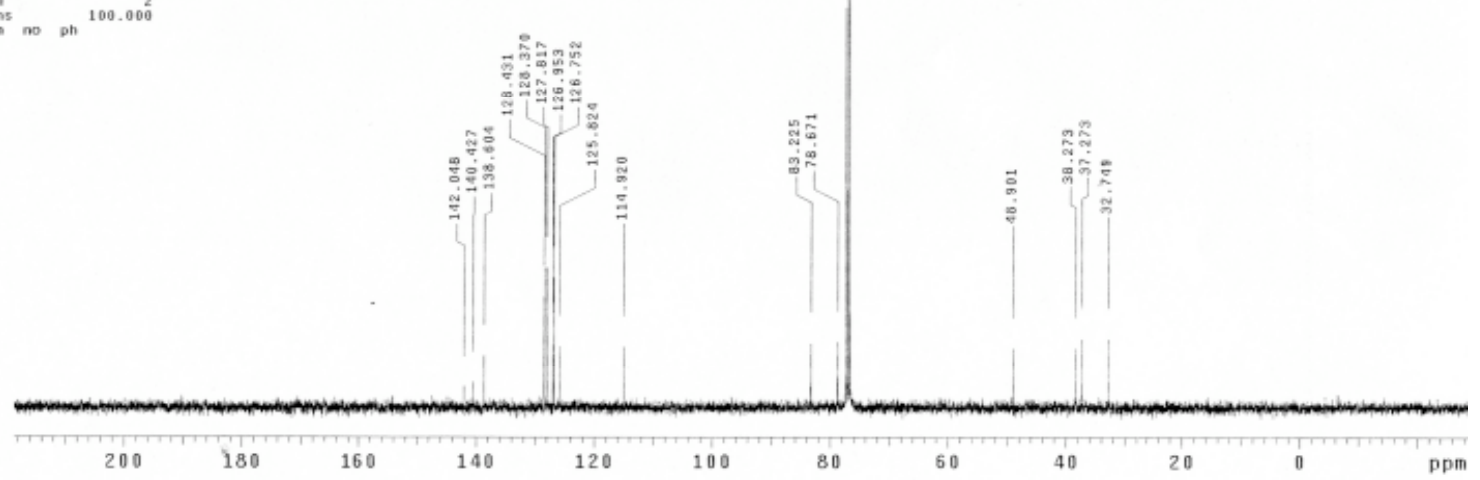


2-heptyl-tetrahydro-5-phenethyl-3-vinylfuran (6)

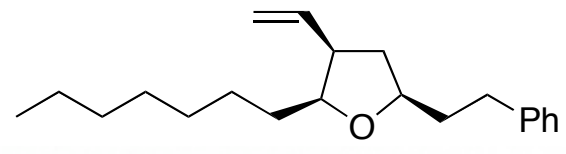

$410 \mathrm{MHz} 10$ in CDC13 (ref, to CDC13 0 $7.25 \mathrm{ppa}$ ), teap $27.0 \mathrm{C} \rightarrow$ astual teap $=27.0 \mathrm{C}$, a 40092 probe
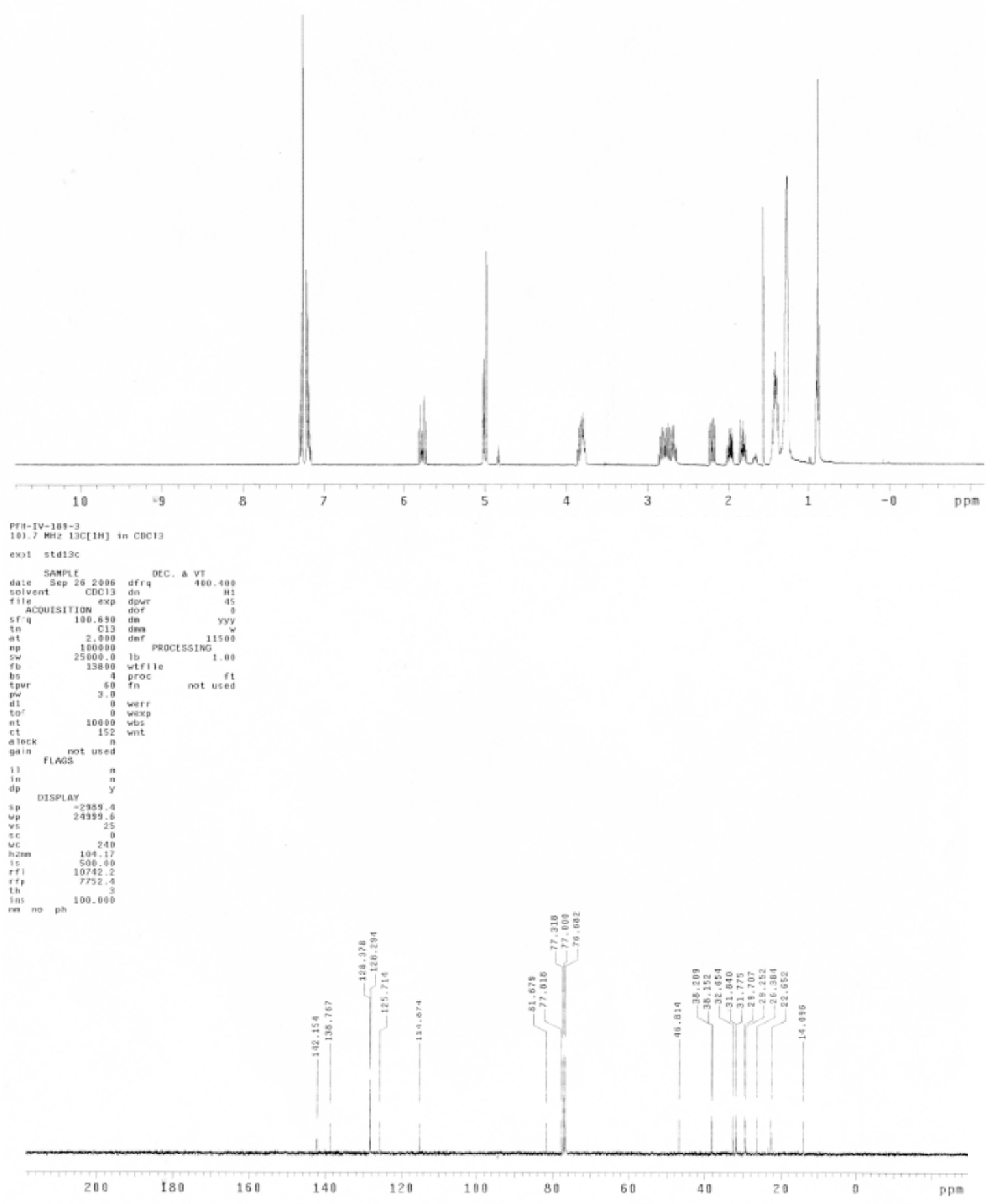
2-((benzyloxy)methyl)-tetrahydro-5-phenyl-3-vinylfuran (7)

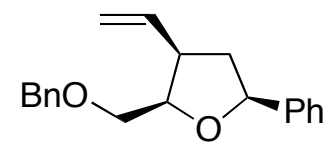

PrH-
$400-125$
$\mathrm{NHz}$

Puise sequence: s

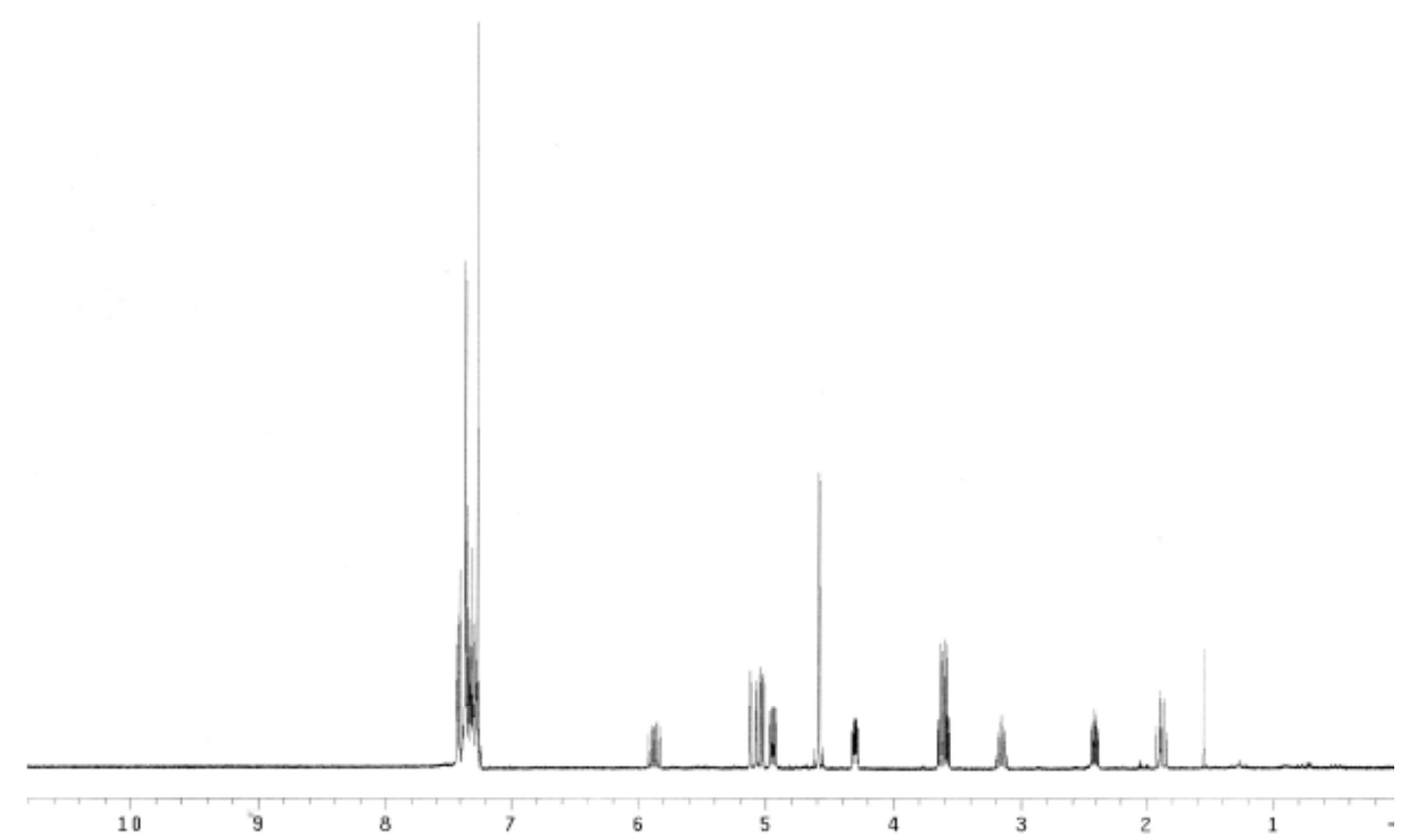

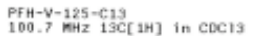

$\exp 2$ std $13 c$
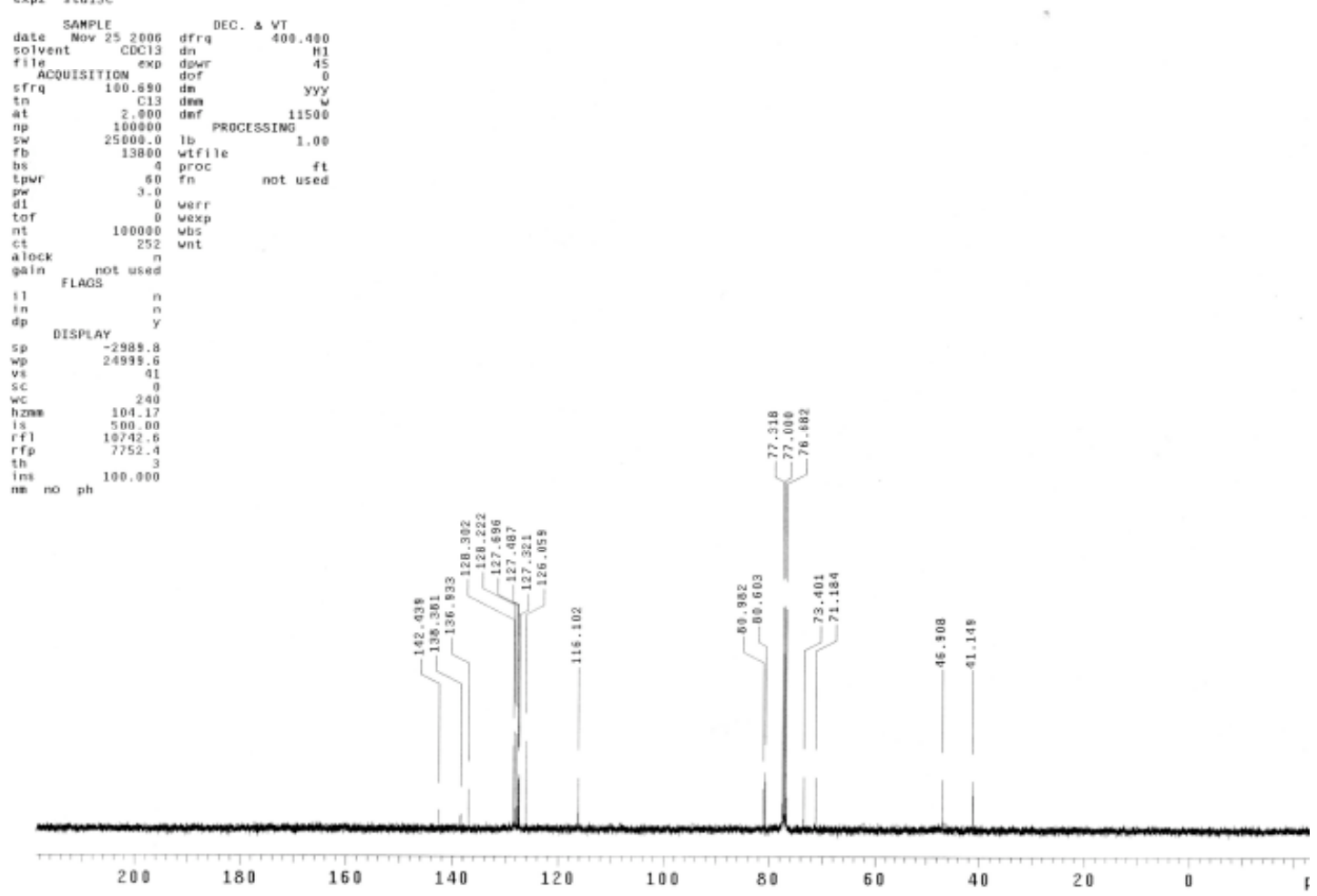
$(2 S, 3 R, 5 R)$-tetrahydro-2-methyl-5-phenethyl-2-propyl-3-vinylfuran (8)
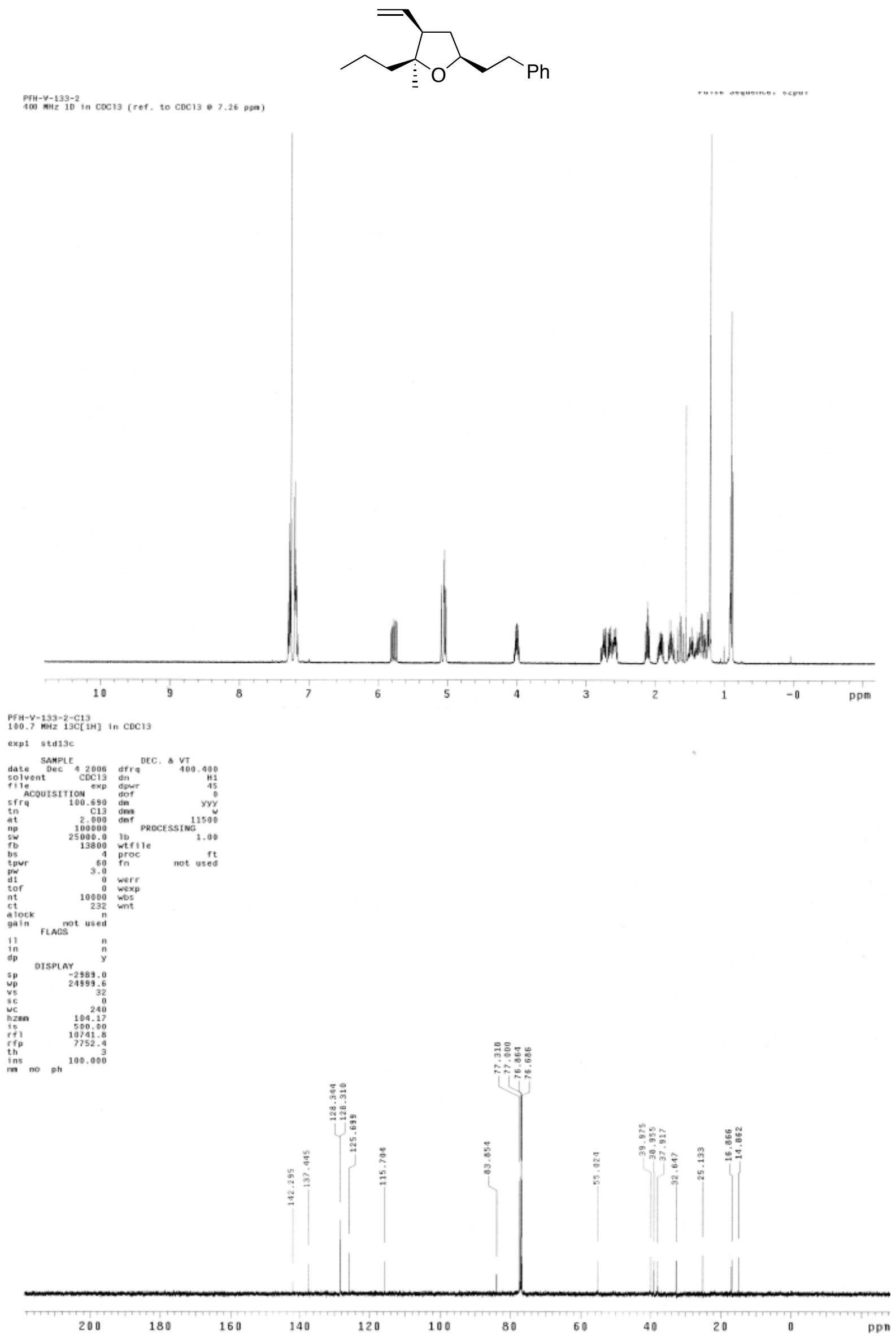

S-38 
2-((benzyloxy)methyl)-tetrahydro-4-methyl-5-phenethyl-3-vinylfuran (21)
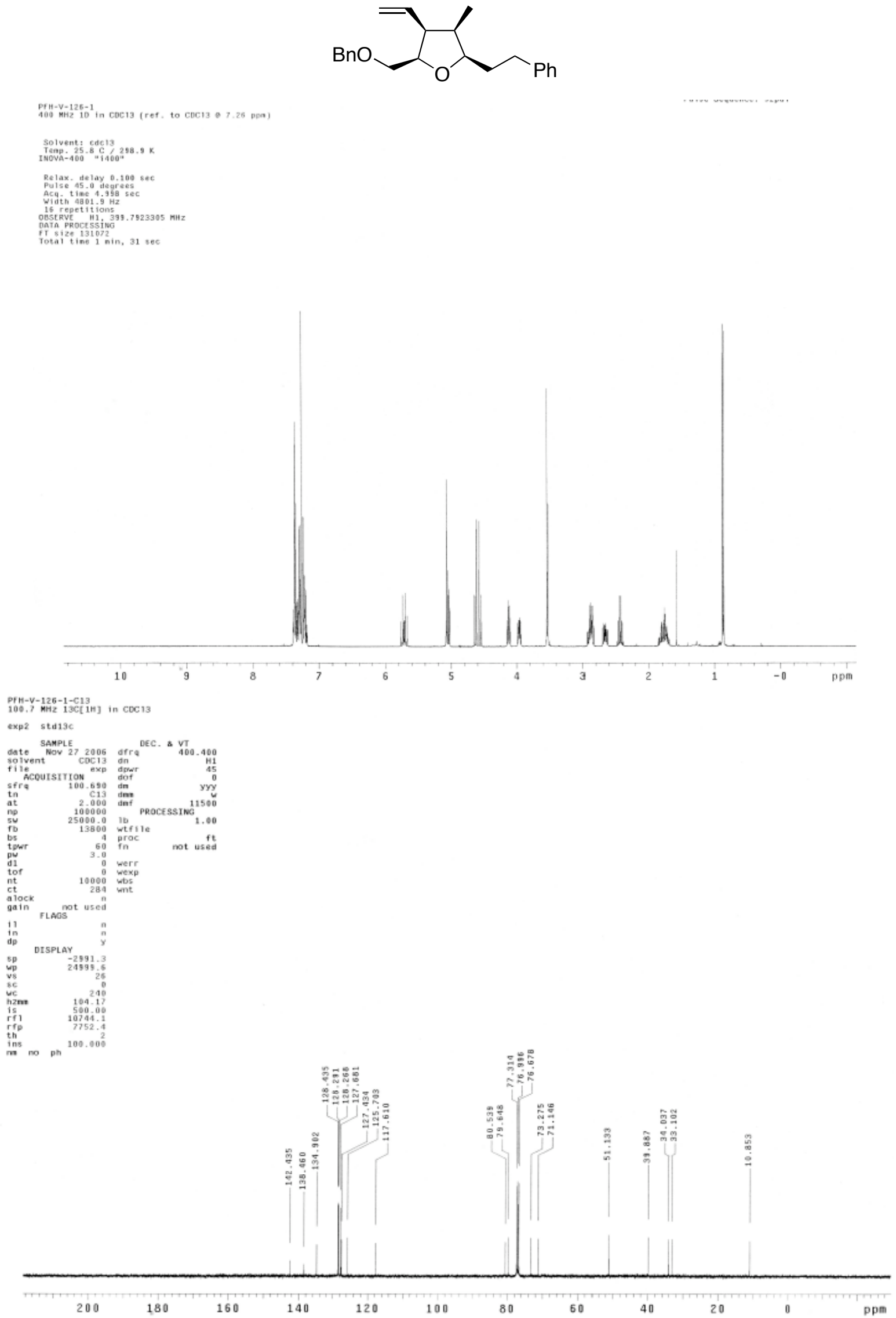

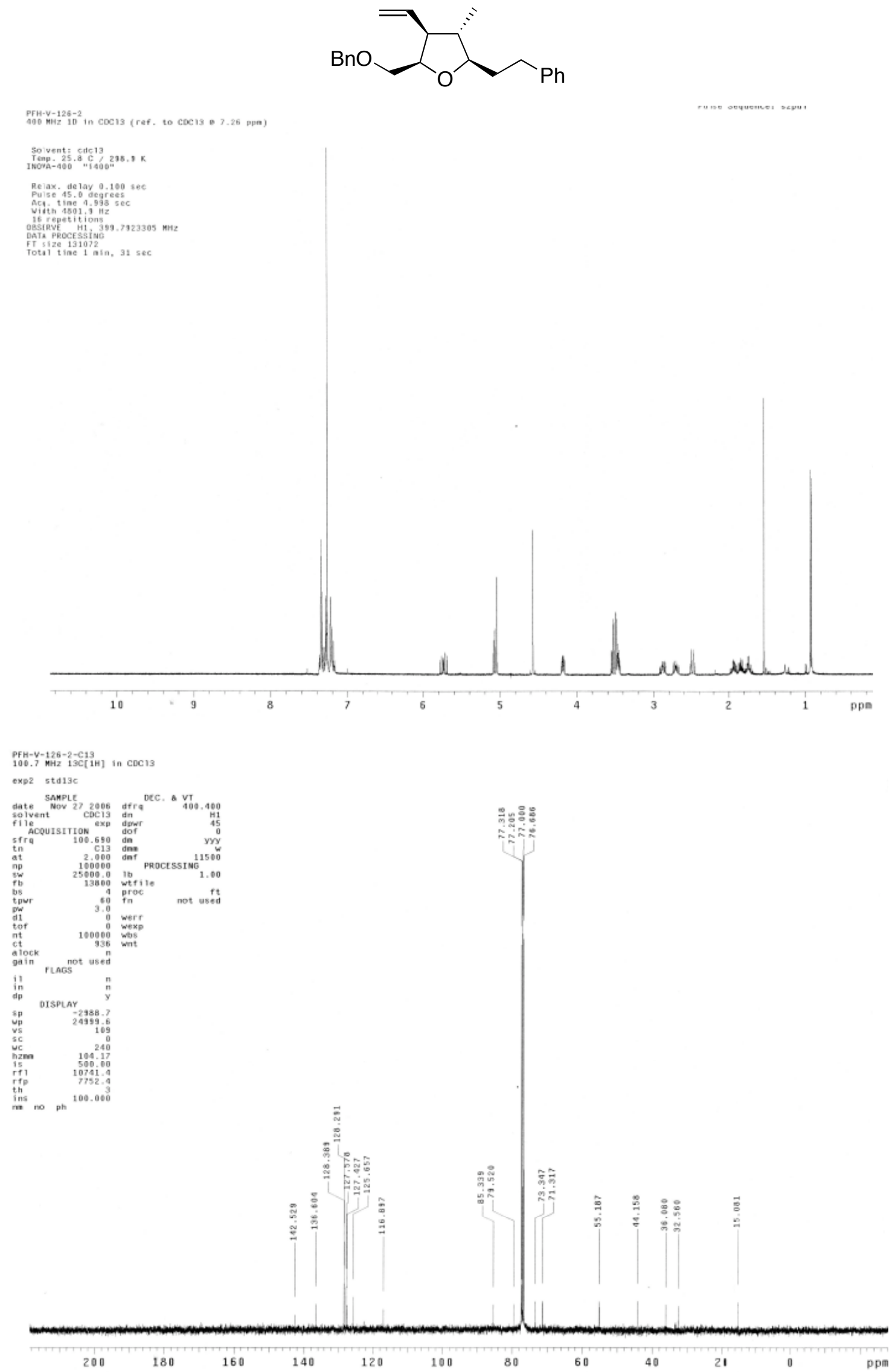


\section{1-(2-((1S,2R)-2-vinylcyclopropyl)ethyl)benzene (9)}
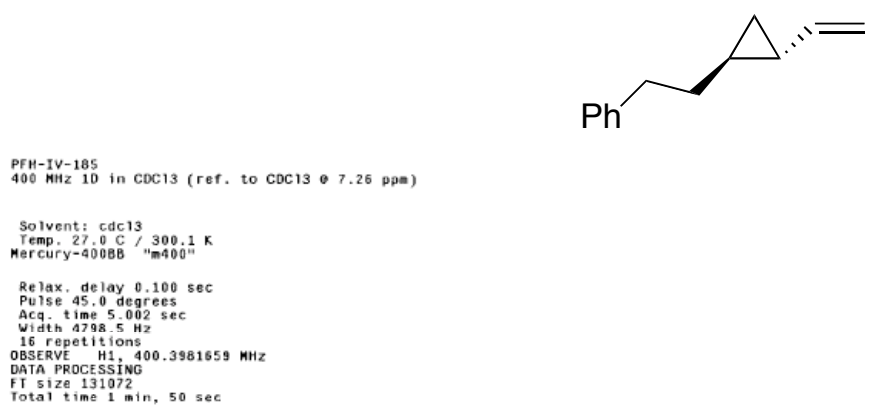

Pulse Sequence: s2pul
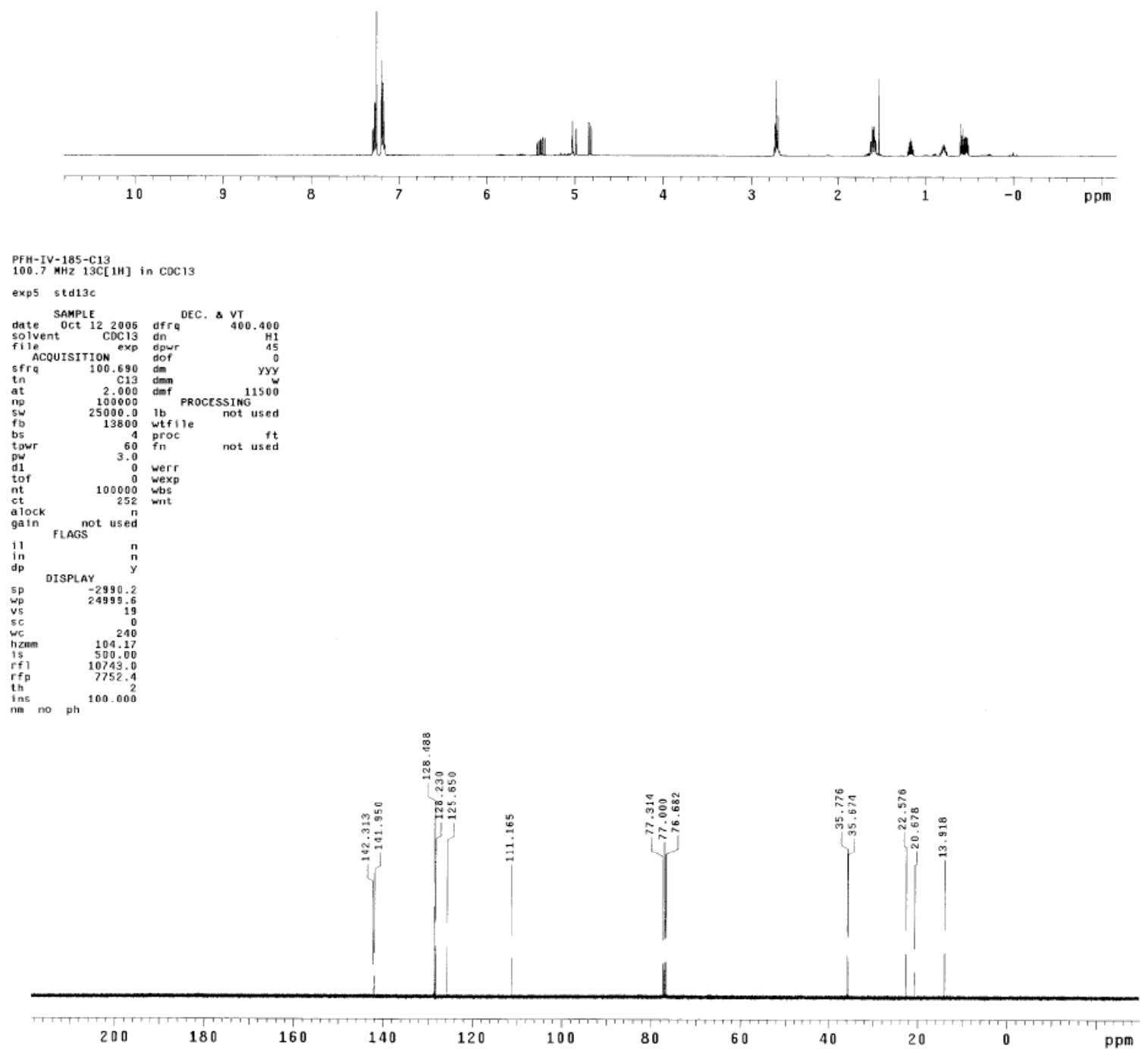
$(1 S, 2 R)$-1-heptyl-2-vinylcyclopropane (10)

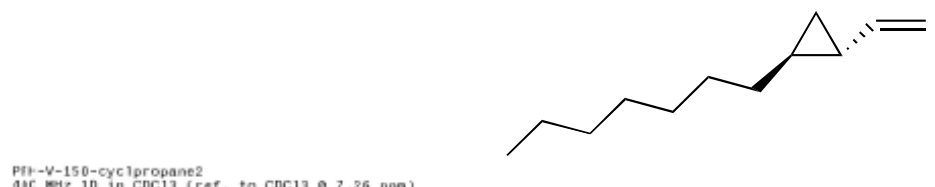

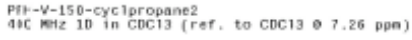

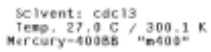

Felax. gelay $0.100 \mathrm{sec}$
P.150 45.0 degrees

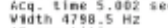

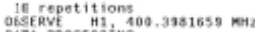

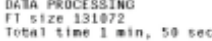

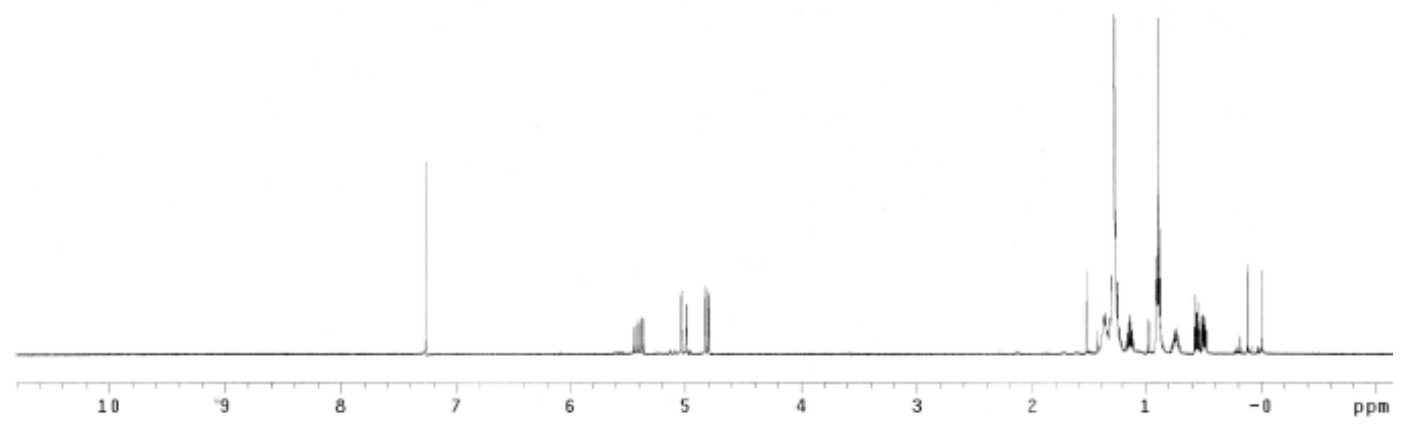

$\mathrm{PFH}-\mathrm{Y}-150-9$
$100.7 \mathrm{nHz}$
$\mathrm{ngC}[1 \mathrm{H}]$

exp7 std13c
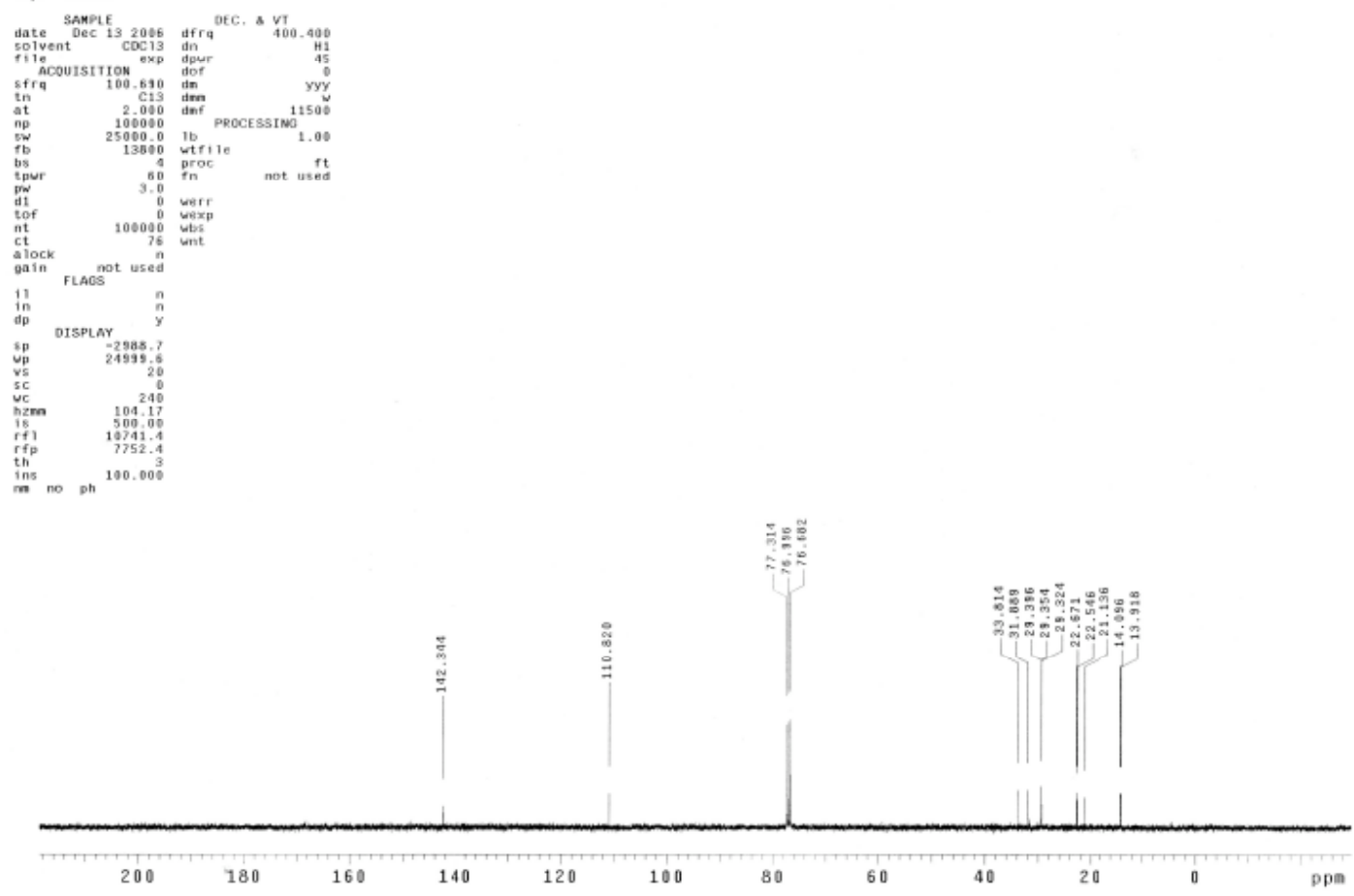
1-(2-((1S,2S,3R)-2-methyl-3-vinylcyclopropyl)ethyl)benzene (23)
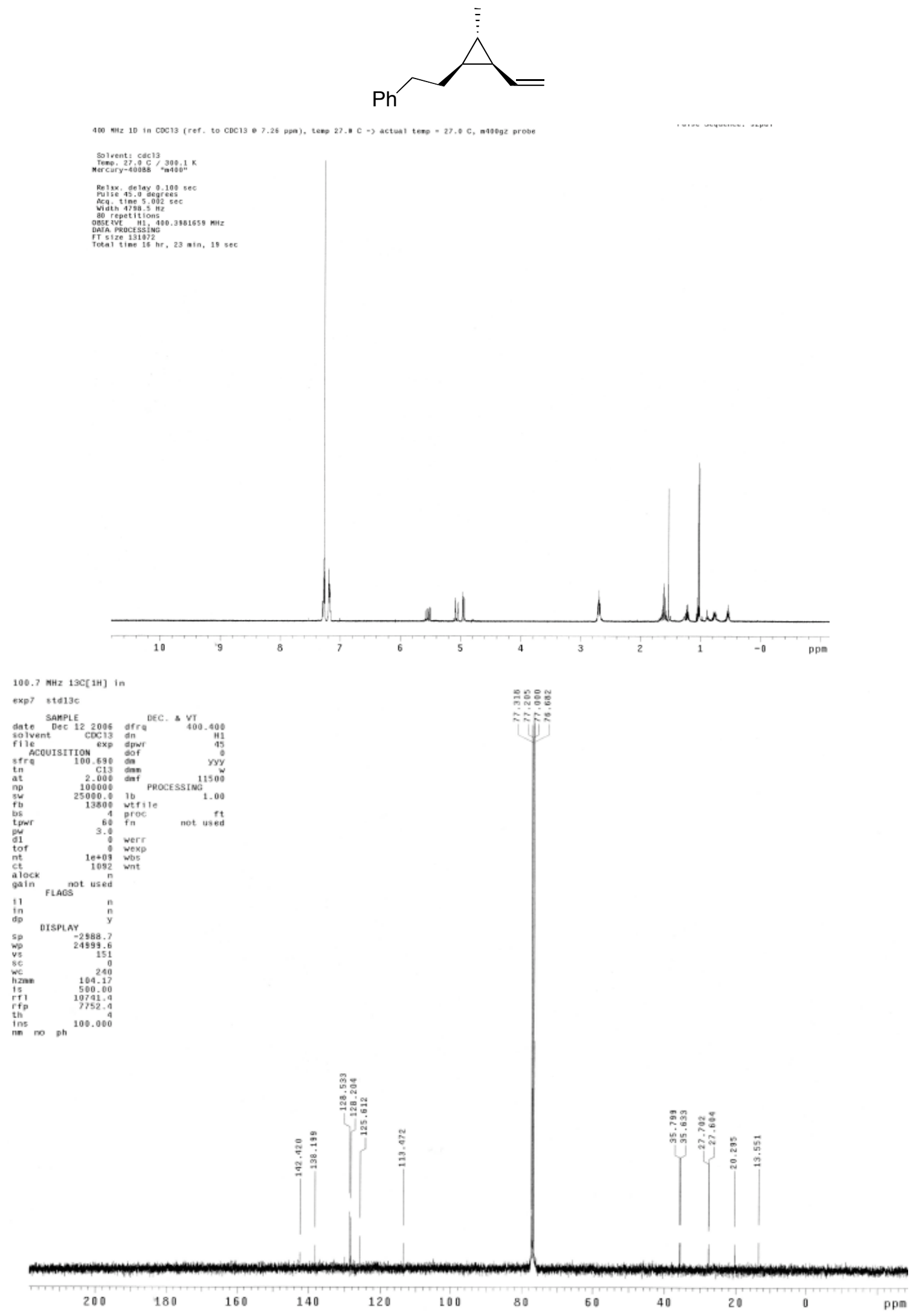


\section{1-methyl-7-vinyl-8-oxa-bicyclo[3.2.1] octane (19)}
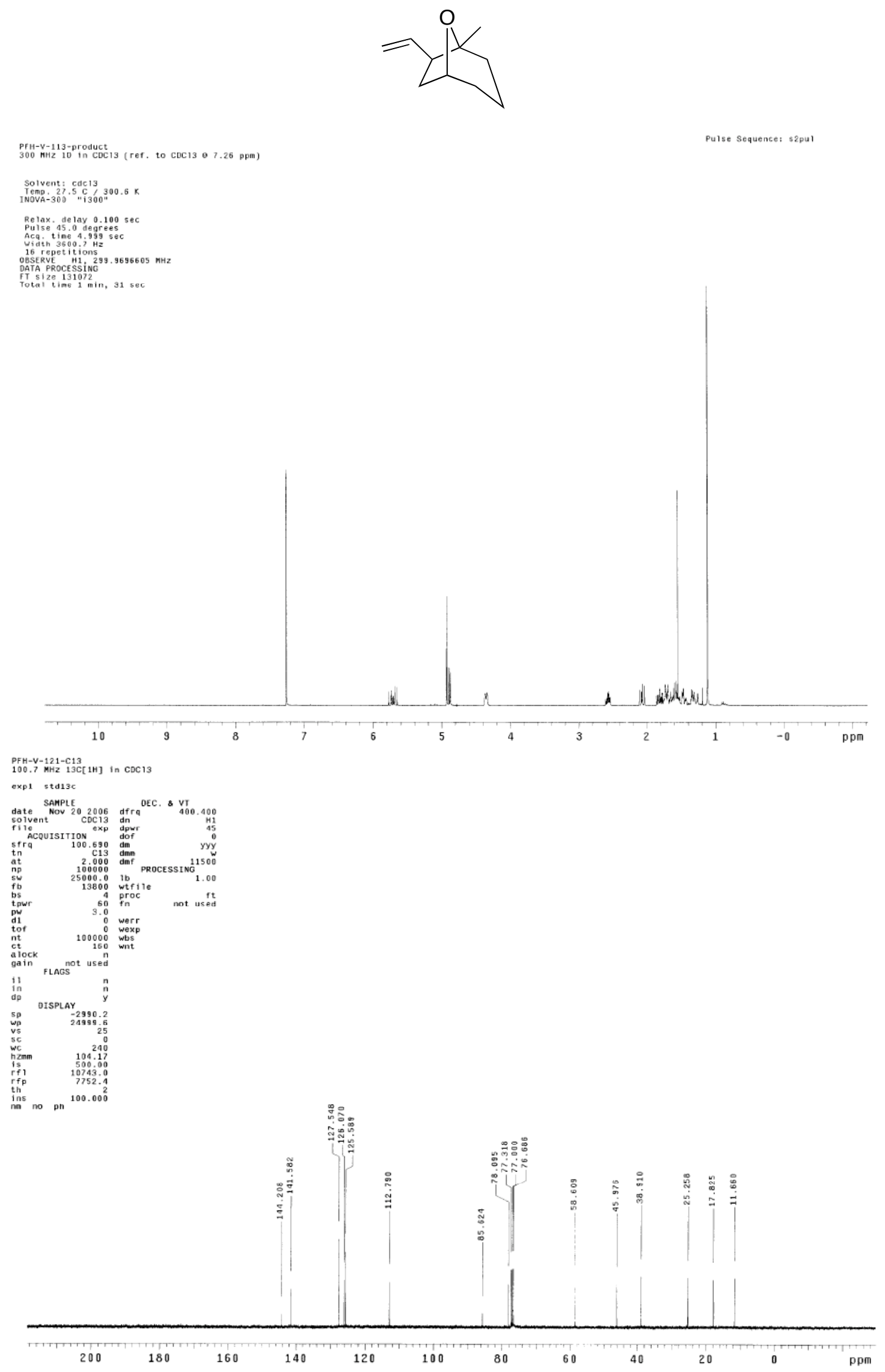
6-methyl-1-phenyl-7-vinyl-8-oxa-bicyclo[3.2.1]octane (20)

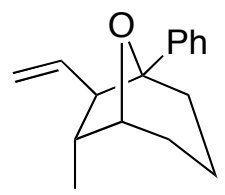

PFH-V - 121
$300 \mathrm{HHz} 10$ in $\mathrm{COCC}_{13}$ (ref. to $\mathrm{COC} 1307.26 \mathrm{ppm}$ )

Pulse Sequence: s2pul

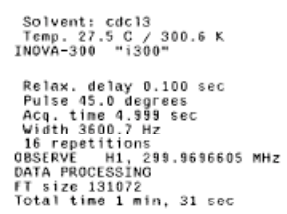

Fr 5 ize 131072
Total time $1 \mathrm{~min}, 31 \mathrm{sec}$

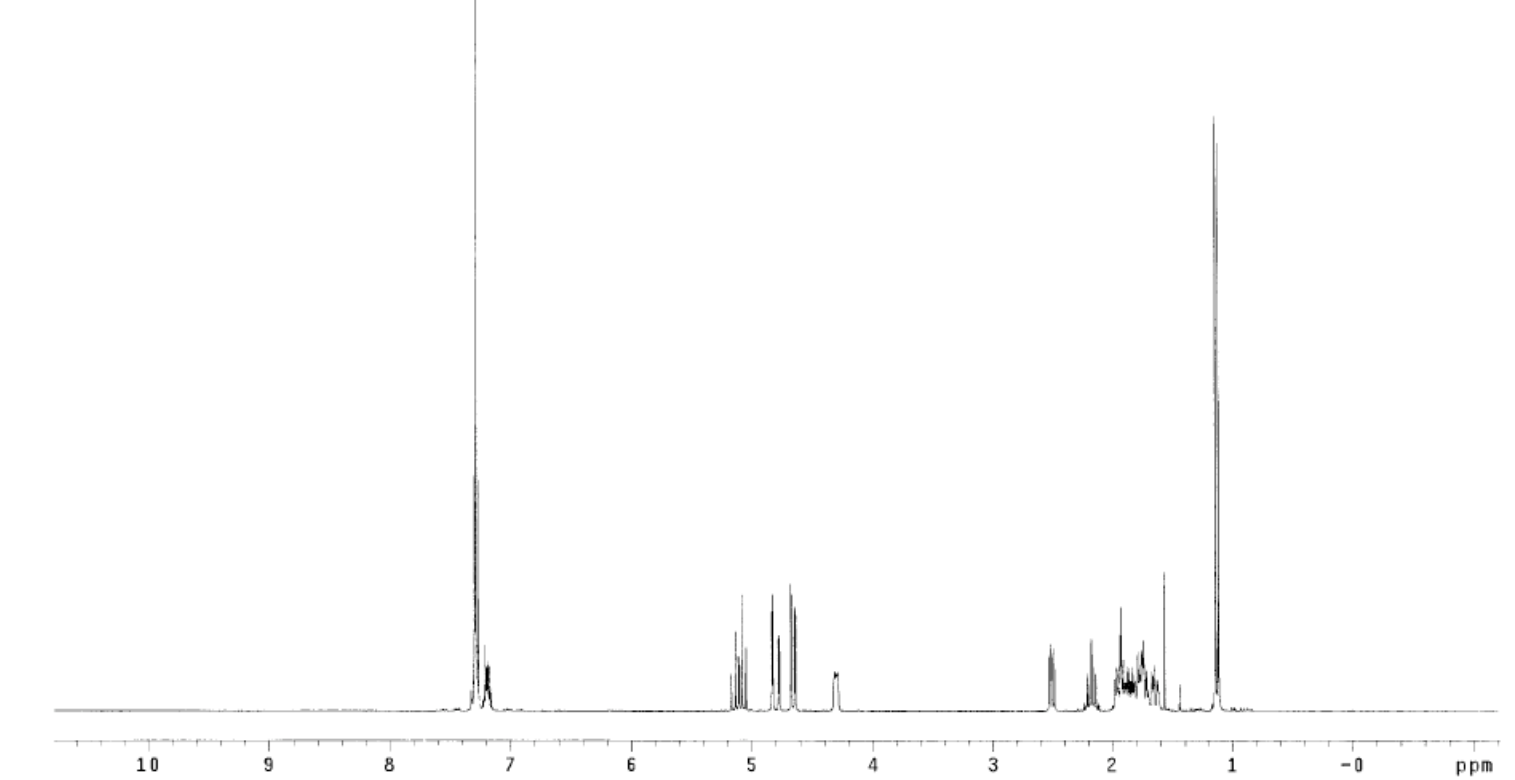

PFH-V $-121-\mathrm{C} 13$
$100.7 \mathrm{MHZ} \mathrm{13C[1H]}$ in $\mathrm{CDC} 13$

exp1 std13c
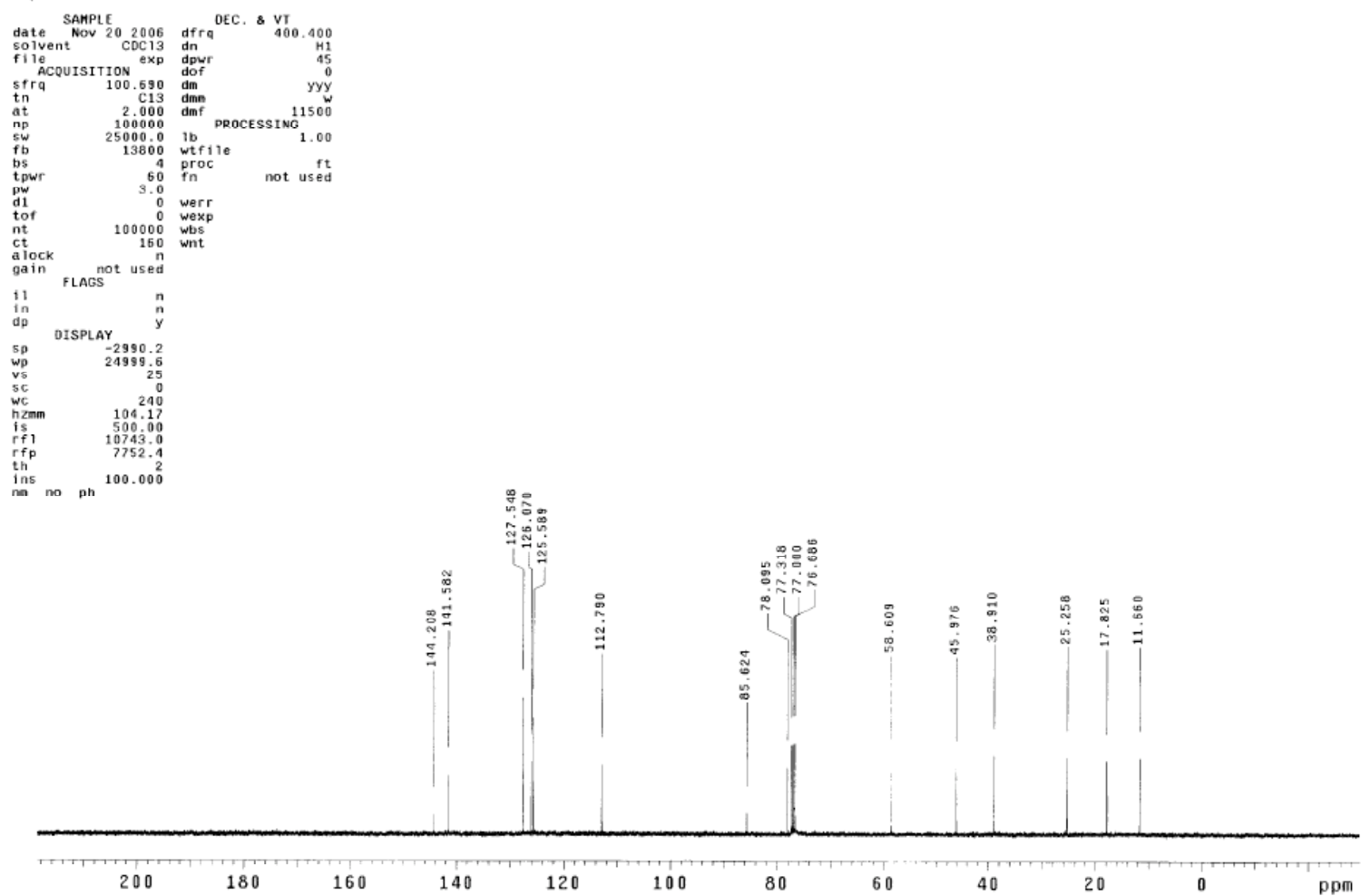


\section{Representative HPLC chromatograms}

\section{Compound 3g}

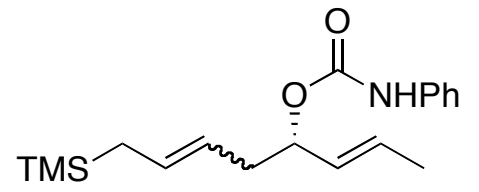

Racemic E/Z mixture sample

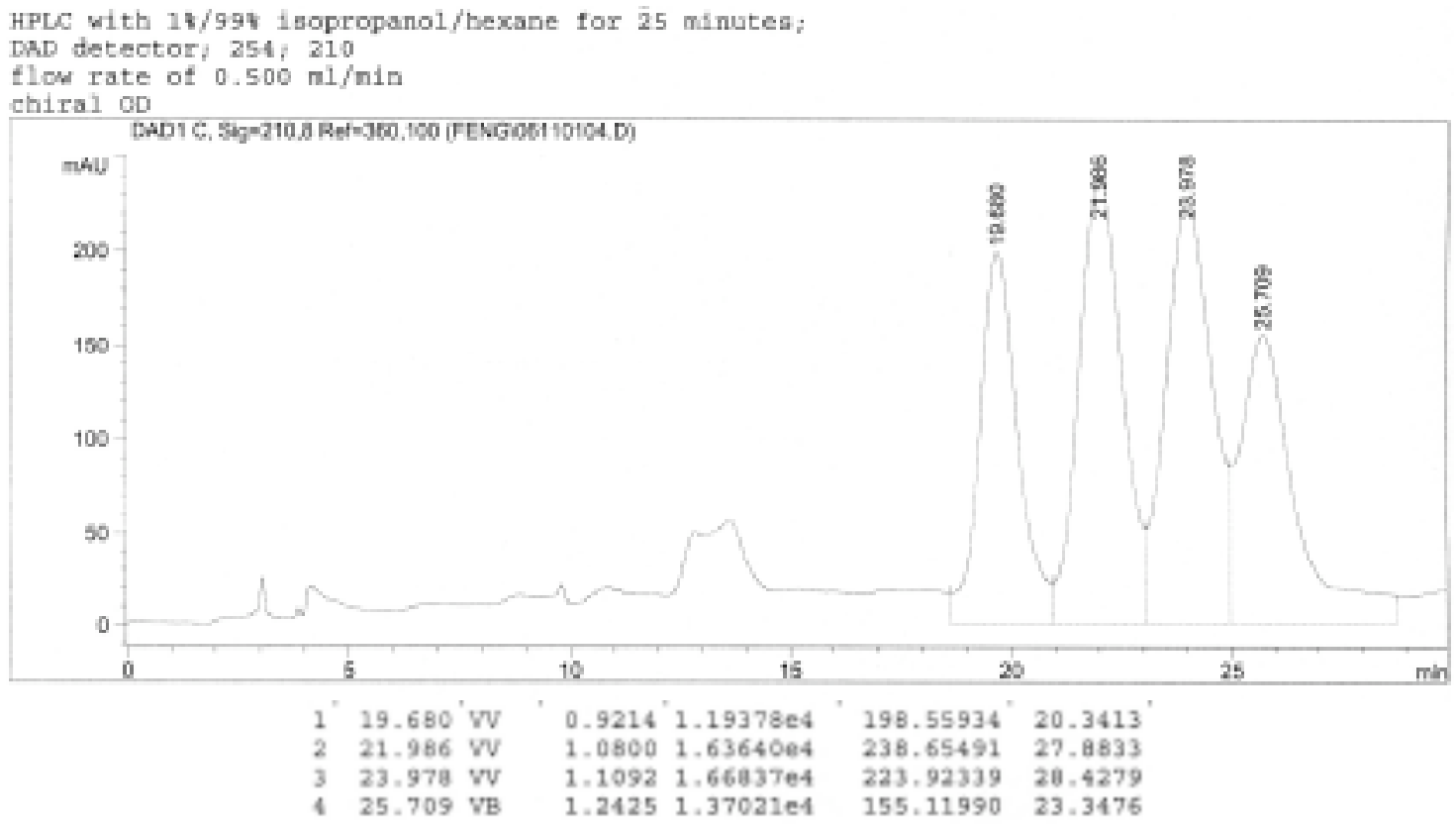

Enantioselective reaction

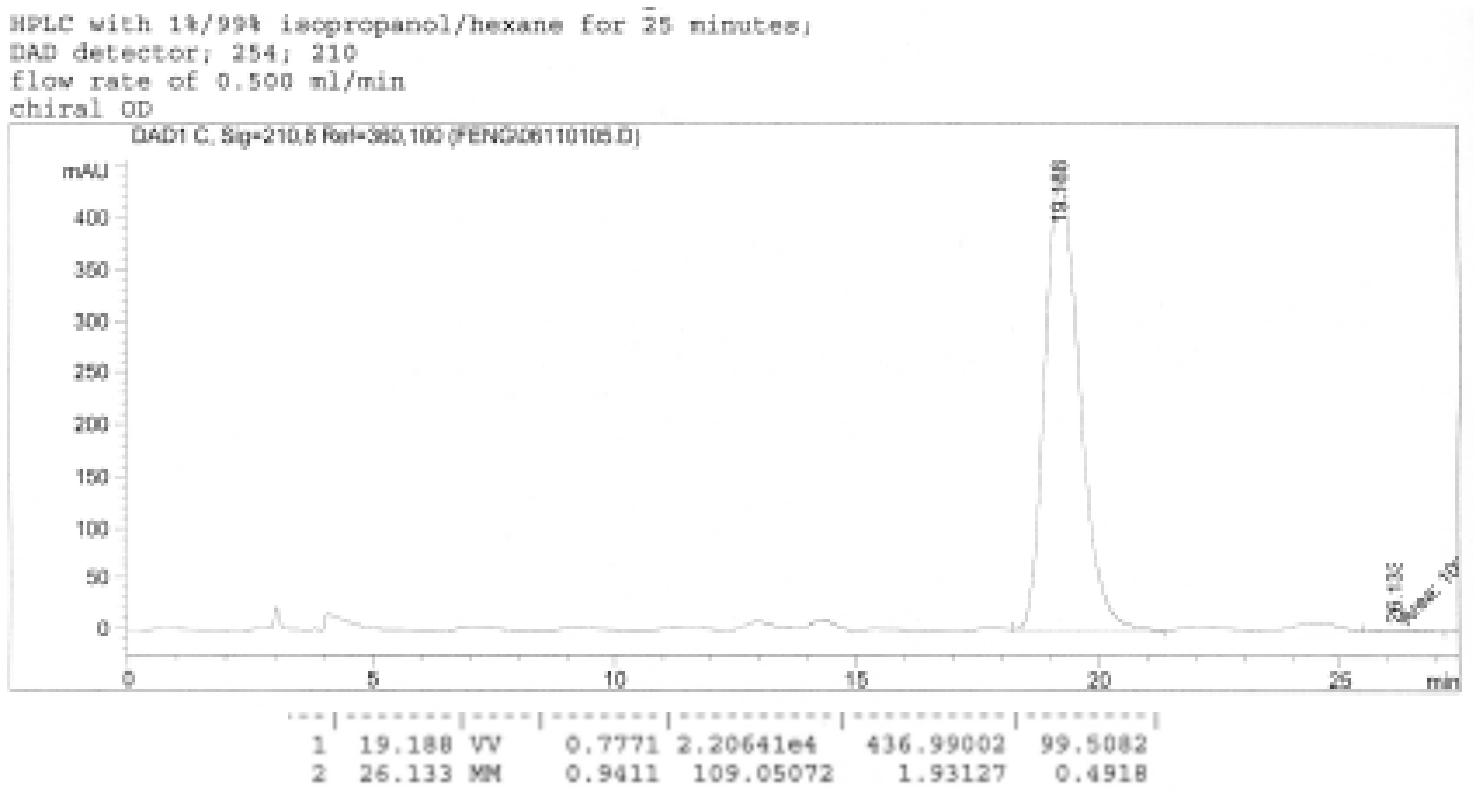




\section{Compound 14}

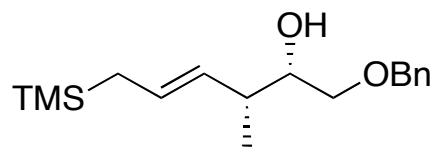

Racemic sample

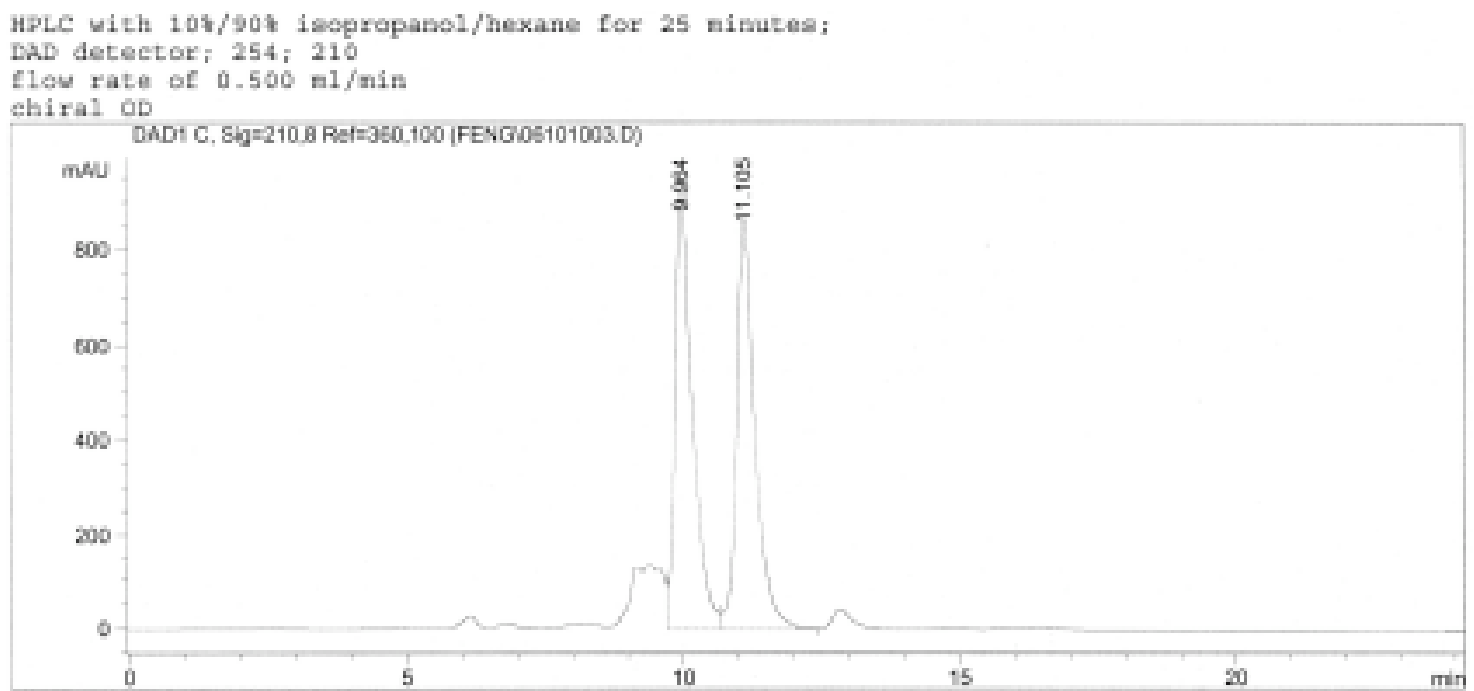

\section{Enantioselective reaction}

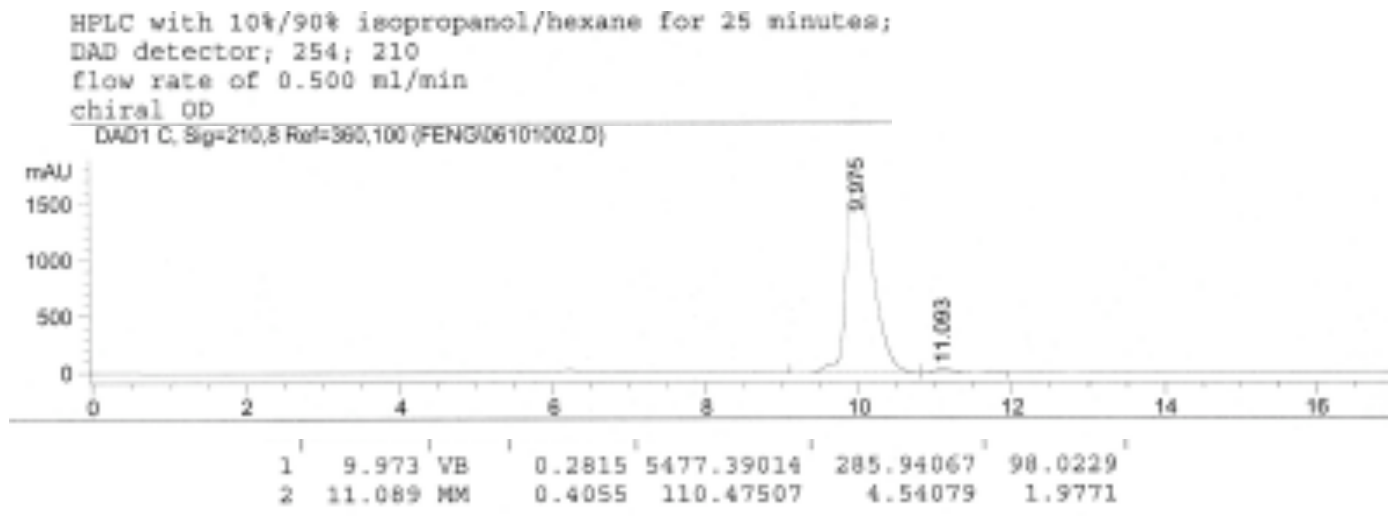




\section{Compound 6}

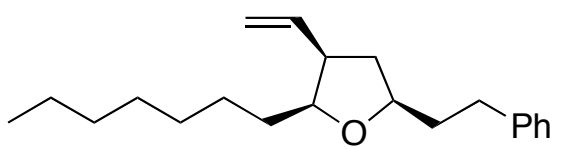

Racemic sample

ADLC with $14 / 994$ iscpropanol/hexane for 25 minutes:

DAD detector; $254 ; 210$

flow rate of $0.500 \mathrm{ml} / \mathrm{min}$

chiral $O D$

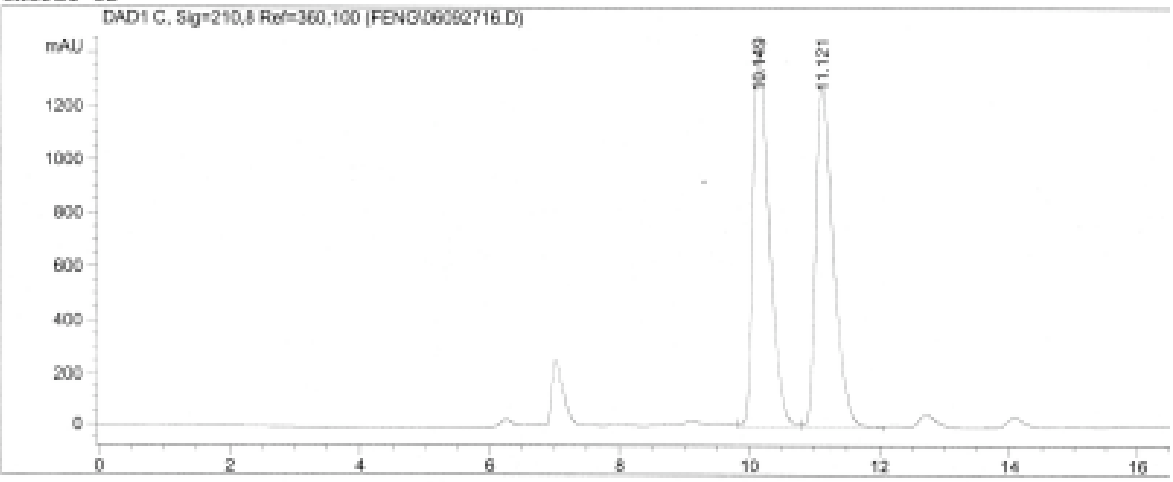

Area Percent Report

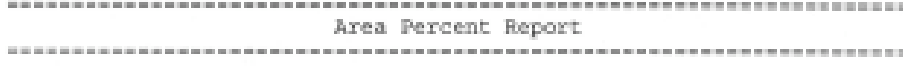

Sorted By t signal

Multiplier 1 1 1.0000

Lse Naltiplier \& Dilution Factor with ISTDE

Signal 1; DaD1 C, Sig=210, B Ref $=360,100$

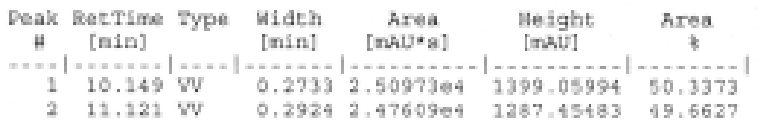

\section{Enantioselective reaction}

HPLC with 14/99\$ iaopropanol/bexane for $\overline{2} 5$ minutes

DAD detector; 254; 210

clow rate of $0.500 \mathrm{n} 1 / \mathrm{min}$

chiral co

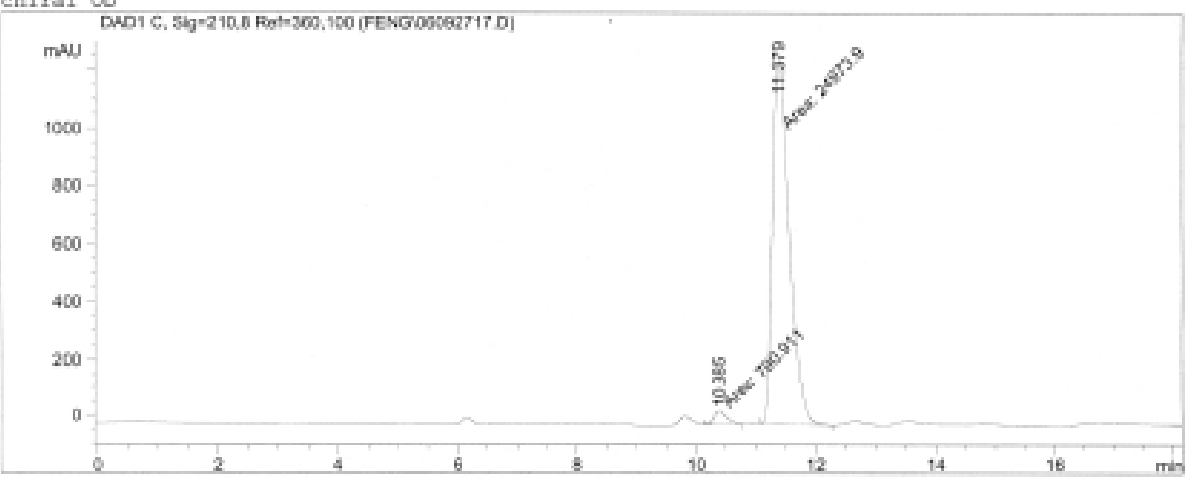

Area Percent Report

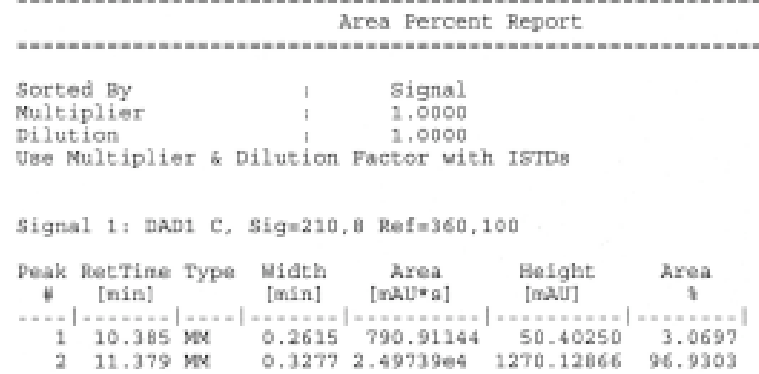




\section{Compound 20}

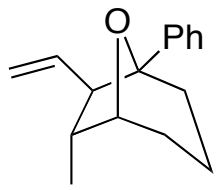

Racemic sample

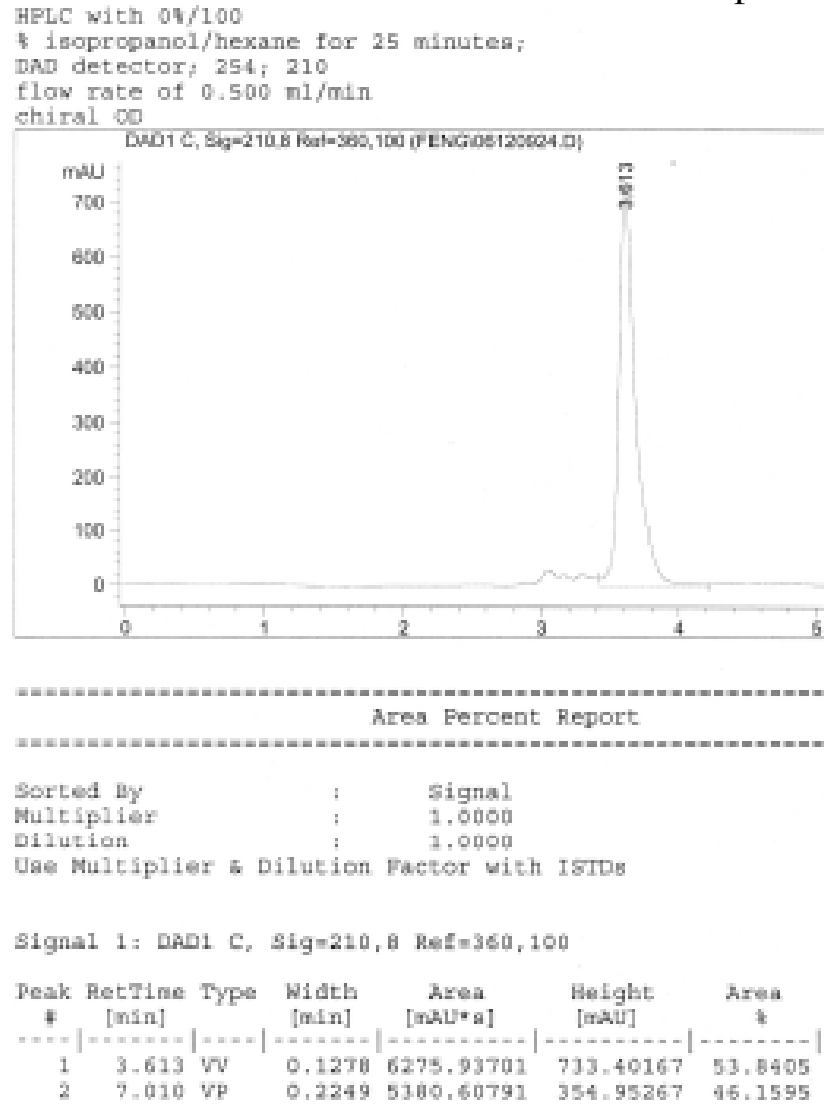

\section{Enantioselective reaction}

KpLe with ov/100

+ ieopropanol/bexane for 25 ninutes

DAD detector; 254; 210

flow rate of $0.500 \mathrm{ml} / \mathrm{min}$

chiral OD
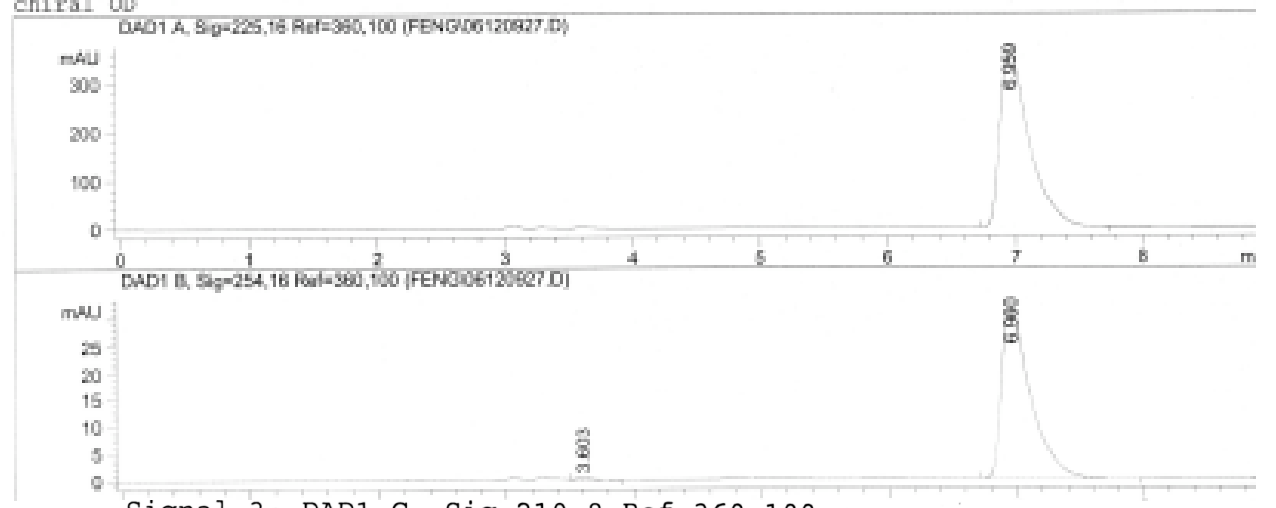

Signal 3: DAD1 C, $S i g=210,8$ Ref $=360,100$

\begin{tabular}{|c|c|c|c|c|c|c|}
\hline $\begin{array}{c}\text { Peak } \\
\quad \#\end{array}$ & $\begin{array}{c}\text { RetTime } \\
\text { [min] }\end{array}$ & Type & $\begin{array}{l}\text { Width } \\
\text { [min] }\end{array}$ & $\begin{array}{c}\text { Area } \\
{[m A U * s]}\end{array}$ & $\begin{array}{l}\text { Height } \\
\text { [mAU] }\end{array}$ & $\begin{array}{c}\text { Area } \\
\frac{\circ}{0}\end{array}$ \\
\hline & & & $\ldots$ & $\ldots-\ldots$ & $\ldots-\cdots-\cdots$ & \\
\hline 1 & 3.608 & VB & 0.1351 & 152.95042 & 16.06600 & 0.5134 \\
\hline 2 & 6.963 & VV & 0.2561 & $2.96395 e 4$ & 1763.06360 & 99.4866 \\
\hline
\end{tabular}




\section{Compound 21}

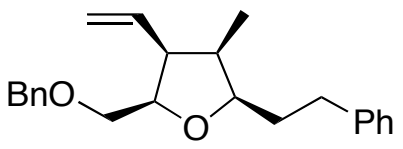

Racemic sample

BDLC with Ov/100

i ieopropanol/hexane for 25 minutes

flow rate of $0.500 \mathrm{ml} / \mathrm{min}$

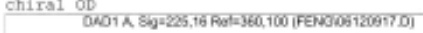
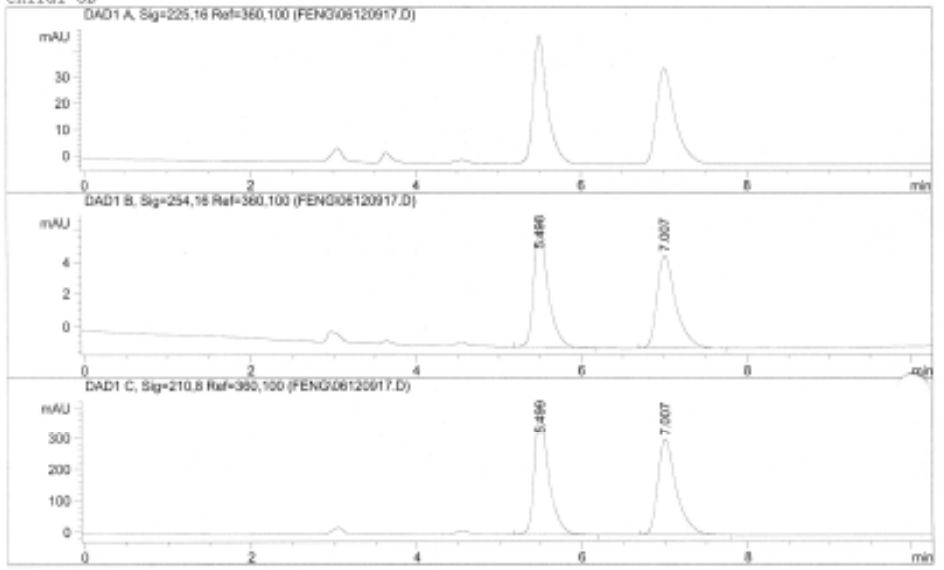

hres Dercent Report

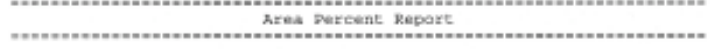

sorted By

Signal
1.0000

Mullution

Use Multiplier a Dilution Pactor vith IsTDa

Signal 1: DAD1 $A$, Sig-225, 16 Ret-360, 100

Signal 2: DAD1 B, 81g=254,16 Ret $=360,100$

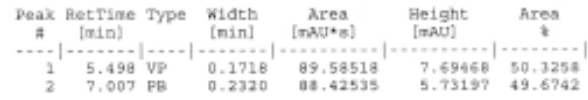

\section{Enantioselective reaction}

HPLC with O5/100

tincproganol/hexane for 25 minutes.

Hab detector; $25 i_{i} 210$

chiral $\infty 0$ of $0.500 \mathrm{ml} / \mathrm{nin}$

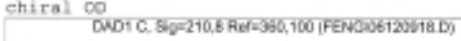

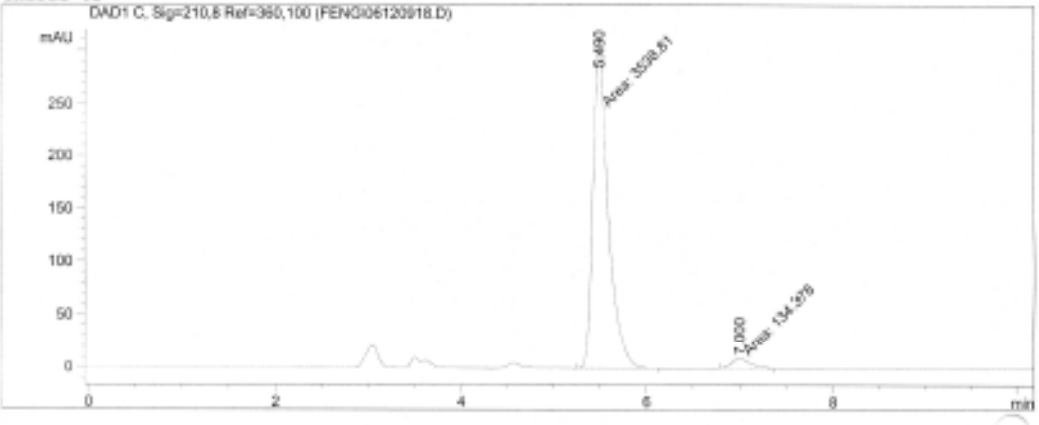

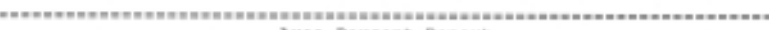

reent leport

Sorted By $\quad: \quad$ Signal

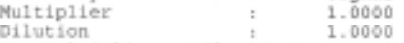

pee Nultiplier \& Dilution Factor with IsTDs

Signsl 1) DAD1 C, sigm210, B Ref $=360,100$

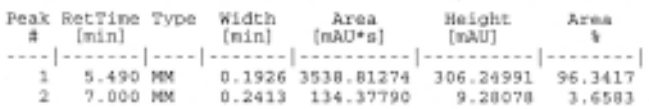


6-methyl-1-phenyl-7-vinyl-8-oxa-bicyclo[3.2.1]octane (20)

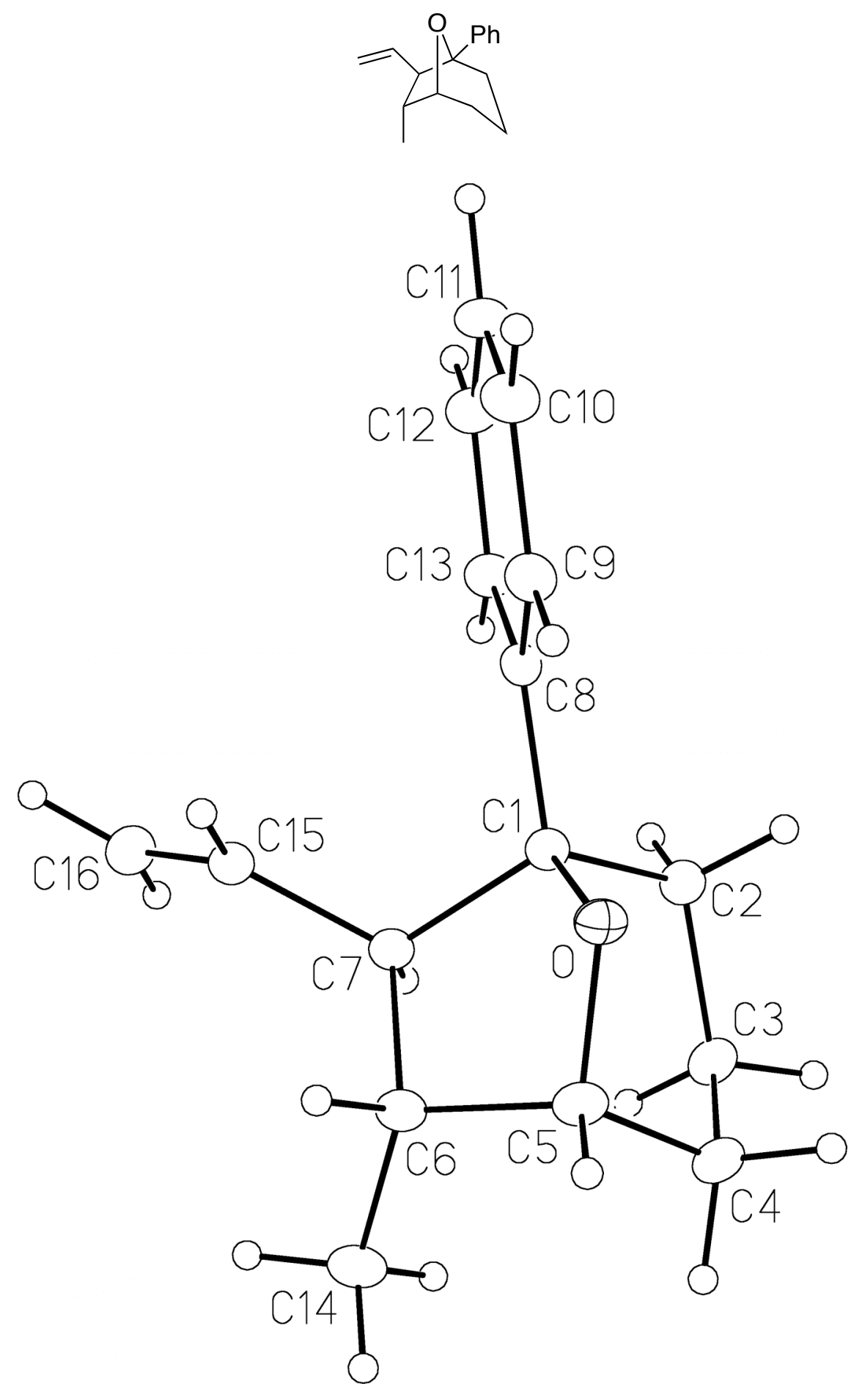




\section{References}

1. Pietruszka, J.; Schone, Niklas. Eur. J. Org. Chem. 2004, 5011-5019.

2. Tsal, D. J. S.; Matteson, D. S. Organometallics 1983, 2, 236-241.

3. Matteson, D. S.; Majumdar, D. Organometallics 1983, 2, 1529-1535.

4. Taylor, R. E.; Engelhardt, F. C.; Schmit, M. J.; Yuan, J. J. Am. Chem. Soc. 2001, 123, 29642969. 